Temporal Cognition: Connecting Subjective Time to Perception, Attention, and Memory

William J. Matthews ${ }^{1}$ and Warren H. Meck ${ }^{2}$

${ }^{1}$ Dept. of Psychology, University of Cambridge, Cambridge, UK

${ }^{2}$ Dept. of Psychology and Neuroscience, Duke University, Durham NC, USA

Running Head: Temporal Cognition

Key Words: time perception; temporal processing; perception; attention; memory

Please address correspondence to either author:

Dr William J. Matthews

Dept. of Psychology

University of Cambridge

Cambridge, CB2 3EB

United Kingdom

Email:wjm22@cam.ac.uk
Dr Warren H. Meck

Dept. of Psychology and Neuroscience

Duke University

Durham NC 27708

USA

Email:meck@psych.duke.edu 


\begin{abstract}
Time is a universal psychological dimension, but time perception has often been studied and discussed in relative isolation. Increasingly, researchers are searching for unifying principles and integrated models that link time perception to other domains. In this review, we survey the links between temporal cognition and other psychological processes. Specifically, we describe how subjective duration is affected by non-temporal stimulus properties (perception), the allocation of processing resources (attention), and past experience with the stimulus (memory). We show that many of these connections instantiate a 'processing principle', according to which perceived time is positively related to perceptual vividity and the ease of information-extraction from the stimulus. This empirical generalization generates testable predictions and provides a starting-point for the development of integrated theoretical frameworks. Our intention is that, by outlining some of the links between temporal cognition and other domains, researchers in the field of timing and time perception will be encouraged to situate their work within broader theoretical frameworks, whilst researchers from other fields will be inspired to apply their insights, techniques, and theorizing to improve our understanding of the representation and judgment of time
\end{abstract}

Running Head: Temporal Cognition

Key Words: time perception; temporal processing; perception; attention; memory 


\section{Temporal Cognition: Connecting Subjective Time to Perception, Attention, and Memory}

Unlike other physical and perceptual dimensions, time is truly ubiquitous: all stimuli and activities have temporal extent, and the perception of time is a universal, continuous experience (Allan, 1979; Buhusi \& Meck, 2005; Grondin, 2001; Lloyd, \& Arstila, 2014; Wittmann, 2013). Correspondingly, the judgment of time is central to virtually all behaviours, from basic tasks like foraging and communication, to uniquely human activities like deciding which stock portfolio to invest in or whether to close a slow-loading webpage. However, unlike other basic dimensions, there is no dedicated sense organ for time. Rather, psychological time is an abstraction, a construct or epiphenomenon of our mind's functioning that somehow infiltrates our conscious experience. That is, we create subjective time in order to explain the coherence of events (Allman, Yin, \& Meck, 2014; Callender, 2010; Grondin, 2001, 2010; Macar \& Vidal, 2009).

In keeping with both its importance and its intellectual challenge, time perception has been intensively studied since the earliest days of experimental psychology (James, 1890 - see Myers, 1972). Some of this research has employed psychophysical or behavioural techniques and focussed on the development of information-processing models; other work has concerned the neural basis of timing, including the effects of pharmacological treatments and the brain regions responsible for time perception. Increasingly, researchers have integrated these lines of inquiry by developing quantitative models of time perception that are grounded in neurobiology; a number of recent papers comprehensively review these contemporary neural and information-processing accounts (see e.g., Allman, Teki, Griffiths, \& Meck, 2014; Grondin, 2010; Merchant, Harrington, \& Meck, 2013; Wittmann, 2013), and several edited books/special issues contain interesting collections of (often empirical) articles (e.g., Grondin, 2008; Merchant \& de Lafuente, 2015; Meck, Doyère, \& Gruart, 2012; Meck \& Ivry, in press; Tucci, Buhusi, Gallistel, \& Meck, 2014; Vatakis \& Allman, 2014; Vatakis, Esposito, Giagkou, Cummins, \& Papadelis, 2011; Vatakis \& Ulrich, 2014). Block and Grondin (2014) provide a helpful "review of recent reviews", and an annotated bibliography is available online (Block \& Hancock, 2013). Readers can also find reviews of how sex differences (Block, Hancock, \& Zakay, 2000; Hancock \& Rausch, 2010), developmental changes (Block, Zakay, \& Hancock, 1999; Droit-Volet, 2012 Droit-Volet, 2012; Droit-Volet \& Zélanti, 2013; McCormack, 2015), age and general intelligence (Bartholomew, Meck, \& Cirulli, 2015), body temperature (Wearden \& Penton-Voak, 1995), emotions (Droit-Volet \& Gil, 2009), and clinical conditions (e.g., Allman \& Meck, 2012; Vatakis \& Allman, 2015) influence human time perception.

The current paper takes a different approach. Rather than surveying the rich theoretical accounts of timing, we focus on how the subjective experience of time is related to other aspects of 
perception and cognition. Specifically, we describe how the apparent duration of a time interval is influenced by three key factors: the non-temporal perceptual properties of the sensory input (which we label 'perception', for convenience); the allocation of processing resources between different stimuli and tasks (attention); and the existence of previously-established mental representations (memory). Our motivation is twofold. First, because time perception is in some ways 'special', it is often studied and modelled in relative isolation from other processes and frameworks (Matthews \& Meck, 2014). We hope that an integrated discussion of the links between subjective duration and other mental processes will encourage time perception researchers to explore links to new domains, and encourage researchers from other fields to identify the contribution that their work might make to time perception research. Second, we hope to show that diverse results from studies of perception, attention, and memory can be integrated within a unifying framework which provides a basis for further theoretical development and empirical work. Our over-arching aim is to encourage cross-disciplinary thinking and to highlight research directions that will contribute to the development of unified theoretical accounts, as presaged by Macar, Pouthas, and Friedman (1992) and Teki, Grube, \& Griffiths (2012).

Our focus is on subjective duration - the perception or judgment of the temporal extent of a stimulus or event. As such, we do not consider other aspects of time perception such as the existence or nature of a "perceptual moment", a psychological "now" that quantizes perceptual experience and that is normally investigated by having people making judgments of simultaneity or temporal order (e.g., Allport, 1968; Stroud, 1955 Shallice, 1964; Poppel, 2009; Kristofferson, 1980; Matthews \& Grondin, 2012). Similarly, we do not consider motor timing or rhythm perception, which commonly treated as distinct from duration judgments (Buonomano \& Laje, 2010; Coull \& Nobre, 2008; Mauk \& Bounomano, 2004; Merchant, Zarco, Pérez, Prado, \& Bartolo, 2011; but see e.g., Ivry \& Hazeltine, 1995).

We focus on the judgment of intervals in the range of a few hundred milliseconds to a few seconds, because these are the most commonly-studied intervals in research on human time perception. Perhaps more importantly, this duration range is the one most widely used in nontemporal studies of perception and cognition, making these intervals most relevant to our goal of encouraging cross-disciplinary integration. It is unclear whether timing over this range rests on a unitary system (e.g., Lewis \& Miall, 2009; Merchant, Zarco, \& Prado, 2008; Rammsayer \& Troche, 2014; Rammsayer \& Ulrich, 2005), or whether distinct processes govern the timing of specific sets of durations, such as those below/above one second (e.g., Buonomano, Bramen, \& Khodadadifar, 2009; Lewis \& Miall, 2003; Rammsayer \& Lima, 1991). The finding that non-temporal manipulations exert differential effects on shorter/longer intervals has been taken to support a multiple-process 
account (e.g., Rammsayer, 1999), so we note cases where the effects that we discuss vary as a function of physical duration.

In Section 1, we introduce some of the key methods and findings of time perception research, and outline an information-processing framework that will help to structure our subsequent discussion. Sections 2, 3, and 4 discuss how subjective time is influenced by nontemporal perception, attention, and memory, respectively. Finally, Section 5 concludes by discussing some implications of the links between subjective time and other mental processes.

Given the breadth of topics under review, our treatment is necessarily somewhat selective. It is not possible to describe or reference every relevant study, but our coverage is based on extensive literature searches and discussion with other researchers (not limited to our collaborators). We have tried as far as possible to describe representative experiments, giving sufficient information about the methods and findings to allow a non-expert to understand what was done and what was found in studies of each type, and we indicate when other studies conflict with a particular finding or body of work. We have also sought as far as possible to be up-to-date in our coverage, describing recent studies that provide new theoretical insights as well as those which replicate long-established effects.

\section{Section 1: Studying Subjective Duration}

In this section we introduce some of the key methods and findings from studies of human temporal judgment so that the tasks and results of subsequent sections may be more easily understood by a broad range of investigators.

\section{Experimental Methods}

Prospective and retrospective judgments. Studies of temporal perception and judgment are divided into those in which the participant knows in advance that a temporal judgment will be required (prospective timing) and those in which the request for a time judgment comes unexpectedly after the stimulus or activity has finished (retrospective timing). Retrospective studies measure 'remembered duration' and usually involve estimating the duration of a task or stimulussequence that lasts tens or hundreds of seconds, with a single trial per participant; they are therefore relatively rare (Block \& Zakay, 1997), and judgments seem to be based on retrieval of the events that took place during the interval (Block, 2003; Block, Hancock, \& Zakay, 2010; Poynter, 1983; 1989). Our focus is on prospective judgments, which tap 'experienced duration' (Block \& Zakay, 1997) and are influenced by the perceptual, attentional, and memory processes that are the emphasis of this review. 
Most prospective studies concern intervals of a few hundred to a few thousand milliseconds. The to-be-timed interval is usually demarcated either by the continuous presentation of a stimulus (e.g., a pure tone or a photograph of a face) or by two very brief 'marker' stimuli with a blank interval in between them (e.g., two 10-ms clicks separated by 1 second); sometimes the interval contains several different stimuli (e.g., a sequence of tones or images), in which case the participant is asked to time the interval between first onset and final offset. Human participants may use counting or other rhythmic activity to assist their judgments. Such strategies become useful for intervals longer than about 1-2 seconds (Grondin et al., 1999; Grondin, Ouellet, \& Roussel, 2004), and a simple exhortation not to count is an effective way to avoid these complications (Rattat \& Droit-Volet, 2011).

Psychophysical tools. Many studies of subjective duration use conventional psychophysical tasks, but researchers also employ methods that are rarely seen in other fields. In conventional studies, the researcher presents two time intervals on each trial: a standard, whose duration is fixed across trials, and a comparison, whose duration varies. The participant indicates which interval was longer (or shorter), and these responses are used to estimate a psychophysical function - e.g., a Weibull, logistic, or cumulative-normal curve relating the size of the comparison interval to the probability that it will be judged longer than the standard. The slope of the curve provides a measure of the variability of temporal representations (flatter = noisier), and the comparison duration which is equally likely to be judged shorter or longer than the standard gives the point of subjective equality (PSE). The PSE indicates how long the comparison stimulus must be to have the same apparent duration as the standard: lower PSEs imply that the subjective experience of the comparison is longer, because it only has to be presented for a short time to seem as long as the standard.

Researchers also use a single interval paradigm in which just one stimulus is presented on each trial and observers classify it as 'short' or 'long' (e.g., Grondin, 2010; Kristofferson, 1980), on the basis that people rapidly develop an implicit standard based on the set of stimuli encountered during the session (Morgan, Watamaniuk, \& McKee, 2000). Other single-interval tasks require the participant to identify which of several previously-presented intervals has been presented (absolute identification), to classify each stimulus on, say, a 7-point scale ranging from 'very short' to 'very long' (category judgment), or to provide a numerical estimate of the duration of the stimulus in seconds or milliseconds (verbal estimation). In these tasks, the mean judgment assigned to a given stimulus provides a measure of its apparent duration.

Temporal production/reproduction. Time-perception researchers also use magnitude production tasks that are relatively rare in other fields. In these tasks, experimenters ask participants 
to produce a given time interval, either by holding down a button for a target duration, by making two responses that are separated by the target duration, or by making a response to terminate the presentation of a stimulus once a target duration has been reached (Mioni, Stablum, McClintock, \& Grondin, 2014). In temporal production tasks, the target interval is verbally-defined (e.g., "2 seconds"); in temporal reproduction tasks a particular duration is presented at the start of the trial participants have to produce an interval of the same extent.

It is worth clarifying what different production responses imply about subjective duration. Consider first a reproduction task in which the experimenter varies the non-temporal properties of the standard interval presented at the start of the trial. A manipulation that expands subjective duration would make the standard seem to last longer, and would lead to longer productions in the second part of the trial. Now consider a variable that is manipulated during the production interval itself. A condition that increases subjective time would mean that the target interval seems to be reached more quickly, leading to a shorter production response than usual. Thus, whether we take a shorter produced duration to indicate an increase or decrease in subjective duration depends on whether the factor of interest was manipulated during the standard- or production-interval stage of the task.

Production tasks are widely used, but it can be hard to separate the contribution of perceptual and motor components (Droit-Volet, 2010), and people may choose to prolong a production/reproduction for reasons that are nothing to do with their representation of duration for example, you might leave an exciting image on-screen for longer than a boring one simply because you enjoy looking at it.

Animal learning paradigms. Finally, time perception researchers have imported methods from studies of animal learning/conditioning. In temporal generalization, the observer is first given several presentations of a standard duration followed by a series of test durations centred on the standard, to which they must respond 'same' or 'different' (e.g., Wearden, 1992). The proportion of 'same' responses for each test stimulus is used to construct a generalization gradient whose width indexes temporal precision and whose peak location indicates accuracy (e.g., Church \& Gibbon, 1982). In temporal bisection, the participant is first given multiple exposures to 'short' and 'long' anchor durations, followed by a test phase in which they judge a set of intermediate durations as being closer to the 'short' or 'long' standard (e.g., Droit-Volet, Brunot, \& Neidenthal, 2004; Penney, Gibbon, \& Meck, 2008). These procedures are unusual in asking participants to 'learn' the standard stimuli at the start of the session, but in other respects they are similar to the conventional psychophysical tasks described above and use the same curve-fitting analysis to estimate of the observer's sensitivity and point of subjective equality. 


\section{The scalar property}

The most widely-discussed findings in time-perception research concern the variability of temporal judgments. Time, like length or loudness, is a prothetic dimension, meaning that sensory analysis consists of establishing the quantity rather than the quality of the stimulation (Stevens, 1957). Like other prothetic dimensions, the just noticeable difference between two times is often a fixed proportion of the reference level (Weber's law), such that the standard deviation of a temporal representation is typically a linear function of its mean. More than this, the distribution functions for temporal judgments are scale invariant, meaning that their statistical properties remain the same at different measurement scales.

Figure 1 plots two illustrative examples. The top row shows data from a temporal generalization experiment in which participants indicated whether various comparison tones matched the duration of a standard (Wearden, Denovan, Fakhri, \& Haworth, 1997). As the standard increases from 2 to 8 seconds, the generalization gradients become more diffuse, but when the data are normalized by dividing the comparison durations by the standard for that block, the curves superimpose (second panel). That is, the generalization gradients are time-scale invariant. Likewise, the bottom row shows data from a bisection task in which participants classified intermediate durations as closer to 'short' or 'long' anchors learned at the start of the session (Allan \& Gibbon, 1991). Each line represents performance with a different pair of anchors; when the stimulus durations are normalized by the bisection point for each curve (right panel), the lines again show good superimposition (with some deviation for the very longest pair of anchors).

This kind of scalar timing has been found in many studies of children, adults, and non-human animals using a range of psychophysical and conditioning tasks (e.g., Brannon, Libertus, Meck, \& Woldorff, 2008; Buhusi et al., 2009; Droit-Volet, 2002; Gibbon, 1977; Gibbon, Malapani, Dale \& Gallistel, 1997; Melgire et al., 2005; Wearden, 1992). Correspondingly, conditions or participant groups which violate scalar timing therefore attract theoretical attention (e.g., Allman \& Meck, 2012; Grondin, 2012, 2014; Gu, Jurkowski, Lake, Malapani, \& Meck, 2015; Kristofferson, 1980; Lejeune \& Wearden, 2006; Penney, Meck, Roberts, Gibbon, \& Erlenmeyer, 2005; Wearden \& Lejeune, 2008). The scalar property is regarded as a central explicandum of time perception (Buhusi \& Oprisan, 2013; Hass \& Durstewitz, 2014; Oprisan \& Buhusi, 2014; Simen, Rivest, Ludvig, Balci, \& Killeen, 2013), and virtually all formal models assume or seek to explain the effect. This is largely because the scalar property constrains the processes by which a mental representation of physical time might be formed. A common way to think about timing is in terms of some kind of counting or accumulation process, and many formal models posit a dedicated internal pacemaker whose 
accumulated pulses form the raw material of subjective time (Figure 4, left panel). For such models, scale invariance provides important constraints on the distribution of pulses. Gibbon's (1977) Scalar Expectancy Theory, for example, makes the neurally-plausible assumption of independent pulses with exponentially-distributed inter-arrival times. However, pulse accumulation is then a Poisson process, meaning that the coefficient of variation $(S D / M)$ grows with the square-root of the mean, contradicting the scalar property and necessitating alternative assumptions about the counting process (e.g., Gibbon, 1992; Gibbon et al., 1984; Treisman, 1963; Simen et al., 2013). Indeed, some researchers have rejected counting as the basis for scalar timing altogether, and have developed models based on, inter alia, the detection of co-incident patterns of activity in neural oscillators (Matell \& Meck, 2000, 2004; Oprisan \& Buhusi, 2014), the neural dynamics of recurrent networks (e.g., Goel \& Buonomano, 2014), the random switching of bistable units between states (Almeida \& Ledberg, 2010), or the continuous monitoring of inflow-outflow systems (Wackermann \& Ehm, 2006).

The scale invariance of temporal judgements is part of a general pattern of many perceptual and mnemonic processes (Bjork \& Whitten, 1974; Brown, Neath, \& Chater, 2007; Moreton \& Ward, 2010; Stewart, Brown, \& Chater, 2005). Indeed, scale invariance has been proposed as a unifying psychological principle that manifests itself in a number of psychophysical 'laws', including the Weber and Stevens laws for perceptual discrimination/estimation, the power law of forgetting, Fitts' law relating movement times to the size and distance of the target, and Pieron's law relating detection reaction-times to stimulus intensity (see Chater \& Brown, 1999). This ubiquity may originate from the statistical structure of the environment, where scale invariant power-law relations are widespread (Chater \& Brown, 1999; Anderson \& Schooler, 1991). In any case, the scalar property can be seen as a commonality between time judgments and other cognitive processes, which is encouraging for the development of integrated accounts. Similarly, time judgments show a range of task, context, and order effects which are found for a wide range of perceptual and nonperceptual continua (e.g., Bausenhart, Dyjas, \& Ulrich, 2014; Brown, McCormack, Smith, \& Stewart, 2005; Dyjas \& Ulrich, 2014; Hellström, 2003; Matthews \& Stewart, 2009a, 2009b; Penney, Brown, \& Wong, 2014) and which have successfully been modelled by over-arching theoretical frameworks (e.g., Brown, McCormack, Smith, \& Stewart, 2005; DeCorte \& Matell, 2015; Dyjas, Bausenhart, \& Ulrich, 2012; Hellström, 1979; Gu \& Meck, 2011; Jazayeri \& Shadlen, 2010; Parducci, 1965; Shi, Church, \& Meck, 2013; Wearden \& Ferrara, 1995).

\section{Introducing the Processing Principle}


This review focuses on the ways in which many non-temporal variables affect subjective time. We survey the various 'temporal illusions' (Eagleman, 2008) in which apparent duration is influenced by basic physical properties, attentional processes, and prior experience with the stimulus. These three topics have been selectively reviewed in the past (Eagleman \& Pariyadath, 2009), but an integrated account of the literature, both within and across these domains, is lacking. Previous discussions covering 'non-temporal' perceptual variables have largely focused on stimulus magnitude (e.g., Bueti \& Walsh, 2009; Walsh, 2003) but, as we shall see, many other factors also affect apparent duration. Likewise, several reviews have described the links between time perception and 'attention', but have focussed on the division of mental effort between a timing task and a separate, non-temporal task - that is, on the importance of 'attending to time' (e.g., Block, 2003; Block \& Gruber, 2014; Block, Hancock, \& Zakay, 2010; S.W. Brown, 2008; Grondin, 2010). We cover this work, but also review broader findings on the effects of directing attention to a stimulus whether endogenously or exogenously, and on the basis of spatial, temporal, or featural cues. And while there have been useful reviews/discussions of 'time perception and memory' (e.g., Block \& Zakay, 2008; Block \& Gruber, 2014), these have dealt with the question of how memory of a stimulus or activity is used to construct a retrospective duration judgment (often using quite long time intervals); studies of how the perceived duration of a presented stimulus varies as a function of whether it has previously been encoded have not been brought together, despite extensive empirical investigation of the roles of sensory, short-term, and long-term memory of subjective time. Finally, while there have been attempts at wider-ranging reviews that summarize results from multiple domains, these have been self-described as brief or selective, and/or relate to a single theoretical position (e.g., Gorea, 2011; Grondin, 2010; Pariyadath \& Eagleman, 2009).

The current review therefore seeks to collate and integrate a wide range of findings from different domains. As we will show, subjective duration is highly labile and shaped by many nontemporal variables. Our primary aim in reviewing these effects is to highlight the deep connections between the experience of time and other perceptuo-cognitive processes, in the hope that this will stimulate cross-disciplinary empirical work and theoretical integration. Rather than simply cataloguing the multitude of behavioural effects, we will seek to show that many of the findings fall under a broad, unifying principle, which we call the processing principle. This is the generalization that the subjective duration of a stimulus is positively related to the strength of its perceptual representation -- the experienced vividness and clarity of the percept, and the ease with which information can be extracted from this representation; correspondingly, the conditions that facilitate perceptual decisions also expand the apparent duration of the stimulus. That is, the 
processes and variables that make a percept subjectively more vivid and objectively easier to identify, categorize, and evaluate also make it seem to last longer.

This theoretical perspective is illustrated in Figure 2; the sketch is intentionally crude, emphasizing the key features of the framework without commitment to specific cognitive or neural accounts of perception. As depicted in the figure, the formation of a percept depends on the confluence of incoming sensory information, the allocation of processing resources, and the existence of prior representations. The overall strength of a percept is modulated by stimulus variables that increase or decrease the input signal, by the intentional or automatic deployment of attention, and by changes to the sensitivity and efficiency of the system resulting from recent or distant past experience with the same inputs. These factors combine to produce a representation that supports processes such as identification, categorization, and the selection of an appropriate action - outputs which can be grouped under the heading 'perceptual decisions' (e.g., Ratcliff \& Smith, 2004).

For 'bottom up' variables (i.e., stimulus properties that shape non-temporal perception), the key issue will be the effective strength of the input signal. This will depend both on the absolute sensory properties of the stimulus itself and on its relationship to the context established by the immediate background and other, recently-encountered items. 'Top down' attention will serve to modulate the sensory input, boosting the effective perceptual strength of some representations at the expense of others on the basis of spatial, temporal, or feature-based expectations or relevance. Finally, pre-existing stimulus representations (i.e., memory) will also modulate the processing of the stimulus to facilitate or impair information-extraction from a given percept. All of these processes shape the overall strength of the percept and ease of information processing; and, in general, they produce corresponding shifts in apparent duration.

It is important to be clear about what the processing principle is, and what it is not. First, it is not (yet) a formal 'model of time perception'; rather, it is an empirical generalization, an abstraction that holds across a diverse body of work examining the links between subjective time and perception, attention, and memory, described in this review. Some of these effects have long been known, but their overarching similarity in terms of perceptual vividity and information-extractions has not previously been highlighted. Second, the processing principle is not a basis for precise quantitative predictions; however, it does suggest useful directions for future research which would contribute towards a more unified account of temporal cognition, and we describe these as we go, whilst also acknowledging situations where the principle does not provide such a useful framework. Finally, the processing principle is not a claim about the specific mechanism by which psychological time is represented or judged. However, it does inform and constrain theories of timing, and speaks 
to recent theoretical developments which could integrate time perception with more general models of cognition. We survey some of these in the final section of the review.

According to the processing principle, the conditions that facilitate perceptual decisions also expand the apparent duration of the stimulus. That is, the processes and variables that make a percept subjectively more vivid and objectively easier to identify and categorize also make it seem to last longer. The remaining sections of this paper describe the connections between subjective duration and non-temporal perception, attention, and memory, and will illustrate how the processing principle provides a useful generalization in each case - as well as noting results which seem to call for an alternative analysis. Irrespective of the utility of the processing principle as a way to conceptualize time perception, the findings that we review - and the commonalities that cut across research areas - mean that subjective time constitutes an important topic for cognitive scientists.

\section{Section 2. Subjective Time and Non-Temporal Properties}

In this section we describe how basic non-temporal properties of the stimulus affect its apparent duration, and discuss over-arching theoretical explanations for these effects. Just as for all perceptual judgments, we can never be sure whether a given variable really affects subjective experience rather than the participant's response strategy - although attempts at disentangling these possibilities are noted below - but in general we will follow widespread practice and use 'judged duration', 'apparent duration', and 'subjective duration' interchangeably, here and throughout the paper. Most of the effects that we describe have been tested in a wide variety of judgment tasks and using observers who are motivated to be accurate; thus, they are not readily dismissed as simple response strategies.

Magnitude Effects. Apparent duration is usually a positive function of the intensity of the sensory signal (Figure 3). Perhaps the most basic example is the finding is that a 'filled interval' (e.g., a continuous tone) is typically judged to be longer than an empty one (e.g., a silent interval demarcated by two brief clicks)). The situation is more complex when longer intervals are used and filled with complicated tasks like card sorting, where the division of attention between the timing and non-timing task are presumed to be important (see Section 3, below). Nonetheless, the filledinterval illusion is a robust feature of prospective timing for stimuli in the milliseconds-to-seconds range and holds across modalities, ages, and designs (e.g., Droit-Volet, 2008; Plourde, Gamache, \& Grondin, 2008; Wearden, Norton, Martin, \& Montford-Bebb, 2007), although it may disappear for very short or very long intervals (Droit-Volet, 2008; Hasuo, Nakajima, \& Ueda, 2011) and be somewhat species-specific (Miki \& Santi, 2005). 
More generally, the subjective duration of a given interval positively correlates with the magnitude of the stimulus. For example, Goldstone, Lhamon, and Sechzer (1978) used a red light emitting diode to define a standard interval of 1 second followed by a variable comparison interval, and had participants judge which was longer using a 5-point response scale. The comparison stimulus was judged longer when it was bright and the standard was $\operatorname{dim}\left(0.8\right.$ and $14.0 \mathrm{~cd} / \mathrm{m}^{2}$, respectively) than when the brightnesses were reversed. Thus, higher intensity illumination increased subjective duration. Goldstone et al. found the same when they varied the loudness of pure tone auditory stimuli. These intensity effects have been replicated using a variety of different tasks (Berglund, Berglund, Ekman, \& Frankenhaeuser, 1969; Goldstone \& Goldfarb, 1964; Matthews et al, 2011; Zelkind, 1973) and generalize to other modalities: Ekman and colleagues found that subjective duration increased as a logarithmic function of the intensity of both electrical and vibrotactile stimulation of the skin (Ekman, Frankenhaeuser , Berglund, \& Waszak, 1969; Ekman, Frankenhaeuser, Levander, and Mellis, 1966). Recently, Lu, Mo, and Hodges (2011) found that heavier weights were judged to last longer than lighter ones in the kilogram range (but not in the gram range, where the intensity differences were presumed to be irrelevant).

These intensity effects can be seen as part of a general principle that subjective duration is positively related to non-temporal magnitude. In one influential study, Xuan, Zhang, He, \& Chen (2007) presented pairs of visual stimuli differing in duration by a factor of 1.25 (e.g., 600 and 750 ms), and had participants indicate which had longer duration. Accuracy was higher when the brief stimulus was dim and the longer stimulus was bright - a congruency effect that replicates the finding that more intense stimuli are judged to last longer. More importantly, Xuan et al. (2007) found the same congruency effect for other magnitudes: empty squares with larger area were judged to last longer than ones with smaller area; arrays consisting of 8 or 9 dots were judged to last longer than those comprising just 1 or 2 , and, higher-valued digits ( 8 and 9 ) were judged to last longer than smaller ones (1 or 2). Similar effects of size and numerosity had been described in earlier studies using different methodologies (e.g., Dormal, Seron, \& Pesenti, 2006; Thomas \& Cantor, 1975), although a recent temporal reproduction study found that the numerosity effect only occurred for males (Hayashi, Valli, \& Carlson, 2013), and Agrillo, Ranpura, and Butterworth (2010) found no effect of numerosity on judgments of duration for tone sequences, albeit using longer intervals (5-13 seconds) than the range we focus on.

Two conceptual issues arise in connection with this work. The first concerns the locus of the effect: does the influence of non-temporal magnitude represent a 'genuine' perceptual confusion or a response strategy? Large objects digits may stimulate 'longer' responses by virtue of overlapping semantic representations, for example, without actually 'seeming' any longer. Yates, Loestscher, and 
Nicholls (2012) found that a conventional comparative judgment task ("which square was presented for longer?") replicated the familiar finding that larger squares were judged to last longer than smaller ones. However, when participants had to classify the durations as 'same' or 'different', the point of subjective equality showed that smaller squares were judged to last longer than large ones. The authors took this to indicate that spatial size biases responses rather than distorting perception. However, recent computational modelling has shown that equality judgments are not well-suited to distinguishing between perceptual and decisional effects (Birngruber, Shröter, \& Ulrich, 2014). In addition, researchers have found that visual size and digit magnitude positively affect responses in temporal reproduction tasks, which are thought to avoid the stimulus-response compatibility and decision-strategy effects that might contaminate comparative judgments (Chang, Tzeng, Hung, \& Wu, 2011; Rammsayer \& Verner, 2014).

A second key point is that subjective duration is influenced by relative, not absolute, stimulus magnitude. Against a high-intensity background it is quiet sounds and dim lights that are judged longer than loud/bright ones (Matthews, Stewart, \& Wearden, 2011). Likewise, a mediumsized circle both looks bigger and is judged to last longer when surrounded by small circles than when surrounded by large ones, despite the physical magnitude remaining unchanged (Ono \& Kawahara, 2007). Similarly, a large shape is only judged to last longer than a small one when the participant sees both in the same session and can explicitly compare their sizes (Gomez \& Robertson, 1979); a recent study also found no effect of digit magnitude when small and large numeric values were presented in separate blocks, but obtained the usual 'larger=longer' result when they were intermingled (Vicario, 2011). Indeed, digit effects were enhanced by adding the suffix 'kilograms' and reduced by adding 'grams' (Lu, Hodges, Zhang, \& Zhang, 2009), indicating a pronounced contextual-dependency for the influence of numeric magnitude.

Magnitude effects have generated interest because they relate to the proposal, discussed below, that there may be a generalized 'magnitude representation' system that encompasses time, space, and number (Walsh, 2003; see also Cordes, Williams, \& Meck, 2007). The finding that numeric magnitude influences duration judgments has generated a particularly large volume of empirical work, partly because of the theoretical interest in origins and mechanisms of numerical cognition (e.g., Buhusi \& Cordes, 2011; Carey, 2009; Haun, Jordan, Vallortigara, \& Clayton, 2010; Lustig, 2011), and researchers have investigated numerosity and digit effects on time perception in people with dyscalculia (e.g., Cappelletti, Freeman, \& Butterworth, 2011), neuropsychological patients (e.g., Cappelletti, Freeman, \& Cipolotti, 2011), and infants (e.g., Allman, Pelphrey, \& Meck, 2012; Lourenco \& Longo, 2010), as well as examining how training in number discrimination influences the effect of numerosity on subjective time (e.g., Javadi \& Aichelburg, 2013). 
Beyond magnitude. The 'larger=longer' idea has generated much interest, but other stimulus properties exhibit equally powerful effects on subjective duration. One of the most robust is sensory modality, with auditory stimuli typically judged longer than visual ones of the same duration. In a representative study, Goldstone and Goldfarb (1964) had participants judge the duration of pure tones or steady lights presented for 0.15-1.95 seconds. The lights were judged shorter than the tones, irrespective of whether judgments were on a 9-point 'social scale' (e.g., 'very much less than one second') or a 'subjective' scale (e.g., 'very, very short'). Notably, each participant judged stimuli from a single modality, so the effect was not reliant on direct comparisons (Wearden, Todd, \& Jones, 2006). This auditory-visual difference has been found repeatedly in children as well as young and older adults (e.g., Droit-Volet, Meck, \& Penney, 2007; Goldstone \& Lhamon, 1974; Harrington, Castillo, Fong, \& Reed, 2011; Lustig \& Meck, 2011; Penney, Gibbon, \& Meck, 2000; Wearden, Edwards, Fakhri, \& Percival, 1998). More recent work has found that vibrotactile stimuli are also judged shorter than tones and similarly to lights (Jones, Poliakoff, \& Wells, 2009).

Apparent duration is also influenced by a wide assortment of other stimulus variables, many of which relate to stimulus movement or change. Moving or flickering visual stimuli are typically judged to last longer than static ones (e.g., Brown, 1995; Kanai, Paffen, Hogendoorn, \& Verstraten, 2006; Lhamon \& Goldstone, 1975). Among dynamic stimuli, faster motion/higher temporal frequencies expand subjective duration relative to lower rates of change, although the effect seems to saturate at high frequencies (e.g., Brown, 1995; Herbst, Javadi, van der Meer, \& Busch, 2013; Kanai et al., 2006) and there is disagreement about whether the key factor is speed or temporal frequency (Kaneko \& Murakami, 2009; Linares \& Gorea, 2015). Subjective time is expanded for looming motion relative to receding motion (Ono \& Kitazawa, 2010), and for point-light displays depicting biological motion relative to displays where the motion is scrambled (Wang \& Jiang, 2012; see also Carrozzo, Moscatelli, \& Lacquaniti, 2010). Of particular note, recent work in which moving stimuli were presented against moving backgrounds has established that subjective duration primarily depends on apparent rather than physical speed (Gorea \& Kim, 2015), mirroring the relativity of the magnitude effects discussed above (see also Orgs, Bestmann, Schuur, \& Haggard, 2011). Likewise, subjective duration is shaped by the perceived speed of a composite pattern rather than by the speed of its components (Yamamoto \& Miura, 2012).

Recent work has also shown that, both in the auditory and visual modalities, stimuli that move or change at a constant rate are judged longer than those which accelerate or decelerate, and decelerating stimuli are typically judged longer than accelerating ones (Binetti, Lecce, \& Doricchi, 2012; Matthews, 2011b, 2013). More generally, however, the effects of regularity/complexity - both of static images and of dynamic stimulus sequences - have been mixed (e.g., Aubry, Giullaume, 
Mogicato, Bergeret, \& Celsis, 2008; Folta-Schoofs, Wolf, Treue, \& Schoofs, 2014; Hogan, 1975; Horr \& Di Luca, 2015; Palumbo, Ogden, Makin, \& Bertamini, 2014; Schiffman \& Bobko, 1974), perhaps because of differing complexity-metrics, judgment tasks, and duration-ranges.

Finally, a collection of other modality-specific perceptual properties influence timing. As examples: for auditory stimuli, higher-pitched tones and speech-sounds seem to be judged longer than lower ones (Brigner, 1988; Cohen, Hansel, \& Sylvester, 1954; Yu, 2010; but see Goldstone \& Goldfarb, 1964, for a null result). For visual stimuli, recent work suggests that red stimuli are judged to last longer than blue ones (Shibasaki \& Masataka, 2014), that static images with implied motion seem longer than those without implied movement (Yamamoto \& Miura, 2012), and that Gabor patches with mid-range spatial-frequencies ( 2 cycles per degree) are judged longer than low ( 0.5 cycles/degree) or high (8 cycles/degree) frequency stimuli (Aaen-Stockdale, Hotchkiss, Heron, \& Whitaker, 2011).

In addition to these heterogeneous effects, time judgments are often affected by 'higher level' variables including emotional significance and familiarity. We discuss such effects in the 'Attention' and 'Memory' sections below.

\section{Theoretical Accounts}

Several theories seek to explain the distorting effects of non-temporal stimulus features on subjective duration. Here we describe the three principle suggestions before outlining an account that seeks to unify the research findings.

Internal-clock models. Several theorists have used the pacemaker-accumulator framework to account for the effects of non-temporal dimensions on subjective time. At the onset of timing, a switch closes and pulses from an internal pacemaker flow into an accumulator; at the end of the timed interval the switch opens and the flow of pulses ends; the accumulated pulses form the representation of duration and the current value is compared against values stored in long-term memory to form a decision - for example, about whether a response will be rewarded (Figure 4, left panel).

In this framework, the non-temporal properties of the stimulus might influence the latency to close or open the switch, producing a constant increase or decrease in its judged duration. Alternatively, the rate of the pacemaker may increase, in which case the effect will become more pronounced as the duration of the stimulus is increased (a 'slope effect'). Wearden et al. (1998) applied this logic to the difference between auditory and visual stimuli by having participants make verbal estimates of tones and lights varying from 77 to $1183 \mathrm{~ms}$. They found a clear slope effect: the 
expanded subjective duration of the tone stimuli became more pronounced at longer durations, consistent with a pacemaker increase. Penney et al. (2000) likewise argued that auditory signals accelerate the pacemaker, although they suggested that this might be because auditory signals are better at holding attention and therefore at keeping the switch closed throughout stimulus presentation.

The effects of non-temporal properties illustrated in Figure 3 all provide examples of slope effects, consistent with an increased rate of accumulation. Importantly, irrespective of whether one adopts a 'clock' framework, these data show that changes in subjective duration are not merely a consequence of changes in onset/offset detection; the same applies to many of the other stimulus and observer variables that we discuss throughout this paper: perception, attention, and memory affect the growth of subjective time, not just the latency to begin/end the timing process, although there are some manipulations that produce a fixed 'intercept effect' (e.g., Grommet et al., 2011; Jones \& Ogden, 2015; Matthews, 2011a, 2011c).

Within the pacemaker framework, some researchers have suggested that non-temporal properties affect the memory- or decision-stage of the internal clock model. Cai and Wang (2014) had participants encode a target duration and then reproduce it with a sustained button press. When the target was defined by a digit and the reproduction interval by a green dot, the magnitude of the digit positively affected reproductions. However, when the stimulus order was reversed such that the target was defined by the dot and the reproduction interval by the number, there was no effect of digit magnitude. This argues against a pacemaker (or switch) account, which predicts an effect whenever a stimulus is timed. Cai and Wang suggest that numeric magnitude biases the decision process when "a digit has the opportunity to co-exist in memory with a perceived duration and to bias the noisy magnitude representation of the duration" (p. 8) - although it is unclear why this wouldn't also apply to the on-going stimulus presented during the reproduction interval. Indeed, Chang et al. (2011) did find an effect of digit size for both the target and reproduced durations, arguing against the data and theory of Cai and Wang (2014); Chang et al.'s effect was independent of physical duration, suggesting a switch effect rather than a pacemaker-rate increase.

The pacemaker-accumulator framework has helped researchers interpret the effects of changes in physical duration and stimulus order. These manipulations have not been applied to many of the dimensions discussed above, but it is already clear that the results are likely to be heterogeneous. More importantly, a major limitation of the pacemaker framework is that makes no predictions (and offers no explanation) regarding which dimensions will affect subjective duration, or of the direction of these effects. 
A common magnitude system. Walsh (2003; Bueti \& Walsh, 2009;) has proposed that time, space, and quantity are represented in a common 'magnitude system' located in the brain's parietal cortex (see also Aagten-Murphy, Iversen, Williams, \& Meck, 2014; Dallal, Yin, Nekovářová, Stuchlík, $\&$ Meck, 2015). Under this view, the influence of numerosity, length, or digit-size on temporal judgments arises because these dimensions share a unified metric - the implication being that the measurement of any one of these dimensions involves measuring the others so that the measurement of duration, for example, is partly based on the measurement of number, spatial extent, numerosity, and so on (Figure 4, top right). This idea can be seen as a particular, neuroanatomically-specific version of the idea that intensity/magnitude on one dimension influences perceived intensity on other dimensions.

In support of this common-metric framework, non-temporal magnitudes interfere with one another as well as with judgments of time. For example, Dormal and Pesenti (2007) found facilitation of numerosity comparisons when number and spatial length were congruent (that is, when the more numerous set of dots were also spread out in a longer spatial array); Hurewitz, Gelman, and Schnitzer (2006) reported that circle size interfered with judgments of numerosity; and Pinel, Piazza, Le Bihan, \& Dehaene (2004) found overlapping neural substrates and behavioural interference between judgments of luminance and stimulus size. Furthermore, patients with damage to the parietal cortex often show deficits both in temporal processing and in spatial and numerical tasks, and neuroimaging studies have found overlapping activity in this area in tasks that require representations of time, number, and other magnitudes (see Bueti \& Walsh, 2009, and Dormal \& Pesenti, 2012, for reviews). For example, one recent study presented participants with pairs of dot patterns and had them discriminate their durations or numerosities (Hayashi et al., 2013). fMRI revealed that both tasks activated a common network that included the intraparietal cortex (IPC); moreover, applying trans-cranial magnetic stimulation (TMS) to the right IPC enhanced the effects of numerosity on a subsequent temporal reproduction task.

There are, however, some limitations. Empirically, the finding that relative rather than absolute magnitudes affect subjective duration suggests that, if there is a 'common magnitude system', it cannot involve a 'hard-wired' mapping between particular magnitudes on different dimensions. Moreover, although there is interference between judgments of time, space, and number, the effects of non-temporal dimensions on subjective duration seem to be much stronger than the reverse. For example, Dormal and Pesenti (2013) presented pairs of dot-sequences that varied in the duration, numerosity, and distance (length) covered by the stimuli. Both length and numerosity interfered with time judgments, but duration had no effect on the discrimination of number or spatial extent. And the aforementioned finding that number and physical area may only 
influence temporal reproductions during the encoding of the target interval is hard to reconcile with a genuinely 'shared metric', because such a metric would imply an unavoidable association of larger magnitudes with longer subjective duration during the unfolding reproduction interval, too.

Perhaps more importantly, the common-metric framework has some theoretical limitations. First, it is unclear which dimensions are represented in the common system. Walsh (2003) originally emphasized number, space, and time, on the basis of their joint importance to actions, although other prothetic dimensions such as luminance and sound intensity have also been mentioned (Bueti \& Walsh, 2009; Walsh, 2003) and many of the perceptual dimensions that affect subjective time cannot obviously be classified as 'magnitudes' at all (e.g., sensory modality, spatial frequency, and tone pitch are not prothetic continua). Moreover, it is not clear what the shared representation at the heart of the theory actually consists of: what does it mean, in terms of information processing, to talk of a 'common metric' for time, space, and quantity? Walsh's (2003) framework does not offer a clear answer, although researchers working in the internal-clock paradigm have examined how a common accumulation mechanism might underlie the representation of time, number, and length (Aagten-Murphy et al., 2014; Droit-Volet, 2010a; Meck \& Church, 1983).

Coding efficiency. Eagleman and Pariyadath (2009) have suggested that subjective duration depends on the efficiency of neural coding. Specifically, stimuli which evoke larger neural responses will be perceived to last longer (Figure 4, bottom right). This idea has primarily been applied to results concerning the effects of stimulus repetition on time perception, discussed below, but Eagleman and Pariyadath point out that many of the non-temporal features which expand apparent duration also evoke larger neural responses. For example, Roitman, Brannon, and Platt (2007) found that increasing the number of dots in a display produced a monotonic increase in the firing rate of neurons in the lateral intraparietal area of monkeys.

The coding efficiency account has a number of advantages. First, it is not restricted to a particular type of non-temporal factor; all sorts of variables, not just 'magnitude', can affect the size of an evoked response. Indeed, the framework provides a way to predict which stimulus variables will affect subjective time, and in what direction, unlike the clock- and common-metric accounts. Second, the framework does not require a monotonic time expansion across changes in the nontemporal variable. Eagleman and Pariyadath (2009) point out that the effect of increasing visual flicker on subjective time saturates at about $8 \mathrm{~Hz}$, and that there is a similar saturation in BOLD response to flicker in the striate cortex. Finally, we saw above that the same physical magnitude can evoke different subjective duration depending on how its perceived size is shaped by the local context (Ono \& Kawahara, 2007). Murray, Boyaci, and Kersten (2006) found that stimuli that are perceived to be larger evoke more activity (a larger cortical area) in V1 even if the actual visual angle 
is the same, so the coding-efficiency view may be able to accommodate the context-dependency of non-temporal effects on apparent duration.

Despite its promise, there are limitations. First, researchers have not yet tested whether the subjective duration of a given stimulus on a particular trial is positively related to the size of the evoked response on that trial. Second, Eagleman and Pariyadath (2009) emphasize the idea that coding efficiency reflects implicit predictions and subjective time, but, as we will see below, the available data argue against a simple link between predictability and apparent duration. Finally, and most importantly, the processing of a stimulus involves multiple pre-cortical and cortical neurons across multiple time-scales; as Eagleman and Pariyadath (2009) note, "we cannot currently determine which neural activity will be critical"; indeed, a given stimulus variable may increase the neural activity for some areas/timepoints and decrease it for others. The richness of neural processing means that it may be futile to search for a simple, macroscopic neural basis for time perception.

\section{Non-temporal Perception and the Processing Principle}

We suggest that the effects of basic stimulus properties on subjective time can usefully be conceptualized as part of the broader processing principle that we outlined above. According to this principle, subjective time is positively related to perceptual clarity and ease of informationextraction, such that conditions which favour vivid representations and efficient perceptual decisionmaking also lead to longer perceived duration. Correspondingly, subjective time depends on the interplay between external stimulus properties and the internal allocation of processing resources and recruitment of existing mental representations.

This perspective is like the common-magnitude approach in emphasizing the perceptual properties that will expand or contract subjective time but, like the coding-efficiency account, construes 'magnitude' effects as a part of a broader principle and makes no claim about the associations between different non-temporal dimensions. Also, unlike both the common-magnitude and coding-efficiency models, this framework is agnostic about the specific neural structures or responses that underlie the distorting effects of non-temporal perception on apparent duration.

Which properties will increase subjective duration? Regarding the effects of basic stimulus properties, the processing principle implies that physical features which boost the strength of the percept will also expand apparent duration. Perceptual clarity is partly determined by the basic intensity of the input - a weak signal is less vivid and leads to poorer information-extraction - but the relational nature of perception means that the over-riding issue is the salience of the stimulus: the extent to which it represents a change or 'stands out' from the background context. In most 
circumstances, stimuli which are louder, brighter, moving, moving faster, more numerous, and so on, represent a bigger change than those which are quieter, dimmer, static, slower-moving, or fewer. Salience therefore encompasses the effects both of magnitude and of other properties such as motion and acceleration. (Stimuli may also be salient because of 'higher level' properties such as emotional or behavioural significance; we discuss these effects in later sections.)

Salience also captures the modulating role of background context. We noted above that increasing the background intensity reverses the effects of brightness and loudness on apparent duration (Matthews et al., 2011), and that the same medium-size disc is judged shorter or longer in duration depending on whether it is surrounded by large or small circles (Ono \& Kawhara, 2007). The relative intensity/size of the item is what determines its distinctiveness and, correspondingly, its apparent duration. Moreover, salience is also shaped by the other stimuli presented in the experiment, and by experience from the 'real world'. For example, the apparent duration of a simple line stimulus is positively related to the difference between its orientation and that of recent lines (Pariyadath \& Eagleman, 2012; Schindel, Rowlands, \& Arnold, 2011). Indeed, factors which expand apparent duration in the 'typical' experiment may reverse when the stimulus is rendered less salient by changing the statistical structure of the local environment. For example, Tse et al. (2004) reversed the usual finding that moving stimuli (e.g., expanding discs) seem to last longer than static ones by making the static items relatively rare (but see van Wassenhove et al., 2008). These effects relate to the broader role of prior experience in subjective time, which we discuss in Section 4.

The subjectivity and context-dependency of salience mean that attempts to predict subjective duration from physical properties must take into account how those properties will be experienced by the observer. As one example, existing studies of tone-pitch effects have reported that high-pitched tones are judged to last longer than low ones (Brigner, 1988; Cohen et al., 1954; $\mathrm{Yu}, 2010)$, but all of these papers compared frequencies where higher-pitched stimuli will also sound louder than low ones (e.g., Moore, 2012). In other words, tone pitch may have been confounded with loudness-based subjective salience. Similarly, Aaen-Stockdale et al. (2011) have noted that the numerous studies in which "tones seem to last longer than lights" all used visual stimuli with low spatial frequencies; with mid-range spatial frequencies, the modality effect reverses. Moreover, the spatial-frequency effect itself may depend on the subjective salience of these stimuli, as shaped by experience with the visual world: The Fourier amplitude spectra for natural images typically have amplitude proportional to 1 spatial frequency (e.g., Field \& Brady, 1997); concentrating energy at mid-range spatial frequencies represents a departure from 'normal' input, producing discomfort and even migraines/epileptic seizures (Fernandez \& Wilkins, 2008; Wilkins et al., 1984). So it is quite 
possible that the mid-range stimuli were especially salient, with a corresponding increase in apparent duration.

Information extraction. Stimulus salience is a key determinant of information-processing, so one implication of the foregoing framework is that the stimulus properties which increase salience and enhance apparent duration will also facilitate the extraction of non-temporal information from the stimulus. This idea has not been directly tested by time perception researchers, but there are suggestive data. For example, brighter stimuli typically seem to last longer than dim ones, and increasing luminance improves digit recall and image recognition by enhancing the rate at which information is extracted from the display (Loftus, 1985; Loftus \& Redruth, 1994). Similarly, visual stimuli are better detected and discriminated when presented more centrally in the visual field, and central presentation also produces longer subjective durations (Kliegl \& Huckauf, 2014). And larger stimuli not only seem to 'last longer' but are preferentially processed in visual search (Proulx, 2010); this effect is driven by the contextually-determined perceived size rather than by physical size (Proulx \& Green, 2010), just as for subjective duration. Likewise, movement, which produces a robust expansion of subjective duration, also enhances the identification of faces (e.g., Lander \& Bruce, 2003), emotions (e.g., Krumhuber, Kappas, \& Manstead, 2013), and scenes (e.g., Matthews, Benjamin \& Osborne, 2007).

Of course, there will be limits to, and conflicts between, these effects. A jet engine has tremendous auditory intensity but this is likely to impair rather than improve the processing of other stimulus properties - and making a visual stimulus progressively larger also shifts more of it away from the centre of the visual field. Nonetheless, the effects of basic stimulus properties on the efficiency of non-temporal information-extraction provides a potentially useful predictor of subjective duration.

\section{The relevance of subjective time to perception researchers}

The connections between subjective time and non-temporal perception have several interesting implications. First, the importance of salience and context to time perception means that researchers who have developed models of these effects in other domains - for example, the 'contrast', 'inhibition', and 'intepretational' accounts reviewed by Todorović (2010) - may be able to apply or adapt them to a range of new phenomena: those relating to subjective time. Equally, these researchers could bolster their theoretical repertoire by importing models which have been developed in the time-perception literature (e.g., the recently-proposed Internal Reference Model of context effects; Dyjas, Bausenhart, \& Ulrich, 2012). This scope for 'cross-fertilization' extends beyond context effects; the fact that perceptual clarity and information extraction are fundamental 
to subjective time means that researchers who work on neural and information-processing accounts of perceptual representation/judgment have rich new pastures in which to apply their models, as we discuss in more detail in the General Discussion.

Secondly, manipulating time perception may provide a novel way to manipulate - and hence to understand -- non-temporal perception. The processing principle means that increased vividity entails increased subjective duration. The converse may also be true: factors that expand apparent duration might enhance unrelated aspects of perceptual processing. For example, presenting an auditory stimulus can lengthen the apparent duration of a concurrent visual item (e.g., Walker \& Scott, 1981); correspondingly, detection of a fixed-duration visual stimulus can be improved by increasing the duration of an accompanying tone "in a similar way as altering the (actual) duration of the visual stimulus" (de Haas, Cecere, Cullen, Driver, \& Romei, 2013) - as if the expansion of apparent duration by the auditory stimulus serves to improve the effective processing time for the visual target. Similarly, looming auditory stimuli (which, as discussed above, increase apparent duration) improve visual orientation discrimination (Leo, Romei, Freeman, Ladavas, \& Driver, 2011) and increase the apparent size and luminance of visual objects (Sutherland, Thut, \& Romei, 2014). These recent results suggest that the processing principle may operate bidirectionally, with increases in subjective duration boosting perceptual salience and information-extraction. Future work could therefore examine (a) whether the processing changes reported in these studies are mediated by changes in apparent duration, and (b) whether the effects generalize, such that perception researchers can modulate the apparent magnitude, clarity, and distinctiveness of a stimulus by altering its apparent duration via manipulation of other factors known to influence subjective time (e.g., amphetamine administration, body temperature, or presentation of a brief click-train presented prior to the item; Lake \& Meck, 2013; Penton-Voak et al., 1996; Wearden \& Penton-Voak, 1995).

Finally, the intimate links between temporal and non-temporal perception suggest new directions for neuroscientific research. The neuroanatomical basis for timing has been extensively researched and discussed, with particular emphasis on the basal ganglia (especially fronto-striatal pathways) and the (pre) supplementary motor area (e.g., Casini \& Vidal, 2011; Coull \& Nobre, 12008; Hinton \& Meck, 2004; Livesey, Wall, \& Smith, 2007; Meck \& Malapani, 2004; Meck, Penney, \& Pouthas, 2008; Pouthas et al., 2005). Although there may be specialized 'timing circuits', the links between temporal and non-temporal perception suggest functional connectivity between these areas and the cortical (and sub-cortical) activity traditionally associated with processing nontemporal properties - connectivity which has not been fully explored, and which provides potentially fertile ground for neuroscientists studying (non-time) perception. A bolder possibility is that the 
timing networks themselves play a direct role in non-temporal perception; there is certainly emerging evidence that the activity of local cortical networks underlies some aspects of subjective time (Johnston, Arnold, \& Nishida, 2006).

\section{Section summary}

Many basic stimulus properties affect subjective duration. These 'bottom up' factors can usefully be conceptualized as those which alter salience and, correspondingly, the clarity of the percept and the ease with which information can be extracted from the stimulus. Subjective duration typically expands with increases in the difference between the stimulus and its context, although the effects of prior experience and the non-linear mapping between physical inputs and perception mean that the relation between physical properties and experienced salience is sometimes hard to predict. In terms of subjective experience, apparent duration is positively related to perceptual vividity - the extent to which one feels that something has happened when the stimulus occurs - and this improved perceptual clarity corresponds to improvements in the efficiency of information processing and perceptual decision-making.

The effects surveyed in this section provide one instantiation of the processing principle, but perceptual clarity and information extraction also depend on the allocation of processing resources and the existence of prior representations. We consider the roles of attention and memory in the next sections.

\section{Section 3. The allocation of processing resources: Subjective time and attention} Attention involves directing limited resources to improve the processing of specific sensory inputs. Both the selective and resource-sharing aspects of attention have important links with temporal perception (e.g., Buhusi \& Meck, 2009; Champagne \& Fortin, 2008; Coull, Vidal, Nazarian, \& Macar, 2004; Lake \& Meck, 2013; Lui, Penney, \& Schirmer, 2011; Nobre \& Coull, 2010; Viau-Quesnel, Gaudreault, Ouellet, \& Fortin, 2014; see Gorea, 20121, for an interesting theoretical perspective. We first discuss work examining how the allocation of attention to a stimulus affects its apparent duration; we then consider the effects of sharing mental resources between timing and other cognitive tasks.

\section{Selective Attention}

Attending to a stimulus improves its perceptual processing by increasing its share of limited processing capacity (see Chun, Golomb, \& Turk-Browne, 2011, and Knudsen, 2007, for reviews and useful taxonomies). Attention can be directed by cues that indicate where, when, or in which 
modality a stimulus will appear; these cues may evoke an intentional, endogenous shift in attention by virtue of their symbolic meaning (e.g., an arrow pointing to the location of a forthcoming target; Posner, 1980), or may trigger automatic, exogenous orienting to a particular time, place, or channel (e.g., a brief flash near to where the target will appear; Jonides, 1981). There are important differences between endogenous and exogenous attention, but both types improve not just the detection but also the perceptual clarity of the target stimulus, acting as a form of gain control that improves stimulus identification and discrimination (Carrasco, 2011; Chun et al., 2011; Hillyard, Vogel, \& Luck, 1998). Stimuli may also be selected for preferential processing by virtue of their physical properties and behavioural relevance, again with improvements in perceptual processing (e.g., Anderson, Laurent, \& Yantis, 2011; Corbetta \& Shulman, 2002; Ruz \& Lupiáñez, 2002; Vuilleumier \& Driver, 2007).

Time perception researchers have deployed many of the experimental paradigms that were developed to study the effects of attention on non-temporal perception. To anticipate: several lines of research show that subjective duration is expanded for stimuli that are the focus of attention.

Endogenous cuing. Mattes and Ulrich (1998) had participants classify the duration of black dots as 'short', 'medium', or 'long'. At the start of each trial, an arrow indicated whether the target would be on the left or right of fixation, with the validity of this cue varying between blocks of trials. The validity of the cue as was varied between blocks of trials, with participants instructed to direct their attention to the cued location. Subjective duration increased with the probability that the target would appear in the attended location; that is, attending to the location of the forthcoming stimulus increased its subjective duration. This effect generalized to a duration discrimination task, and was replicated by Enns, Brehaut, and Shore (1999) who established that the cuing effect diminished as the cue-target interval increased from 100 to $1600 \mathrm{~ms}$, that it generalized judging the duration of a gap in an otherwise continuous light stimulus, and that it was not due to the faster onset-detection of stimuli at cued locations.

The effect of endogenous cuing is not limited to spatial orienting. Mattes and Ulrich (1998) also had participants judge the duration of auditory (pure tone) and visual (illuminated LED) stimuli following pre-cues which signalled the modality of the forthcoming signal. Subjective duration increased proportional to the allocation of attention to the appropriate channel, an effect which generalized across durations (from tens to hundreds of milliseconds) and time-judgment tasks (category rating and paired-comparison).

Exogenous spatial cuing. Subjective duration is also affected by involuntary shifts of attention. Yeshurun and Marom (2008) had participants compared the duration of two discs presented to the left or right of central fixation. One disk was preceded by a neutral cue -- a bar 
above both possible target locations. The other disc was preceded by a spatial cue - a bar above one above just one of the two locations where the disk could appear. This cue was uninformative (the target was equally likely to appear in the cued and uncued locations). Nonetheless, when the disk appeared in the cued location its subjective duration was expanded relative to the neutrally-cued disk, showing that involuntary orienting of spatial attention expands the subjective duration of stimuli at the attended location.

Yeshurun and Marom (2008) found this effect only for brief durations (c. $100 \mathrm{~ms}$ ) and Chen and O'Neill (2001) actually reported the opposite effect of exogenous spatial cues. However, a comprehensive series of experiments by Seifried and Ulrich (2011) established expanded subjective duration for stimuli at cued locations using a range of cue-types and temporal judgment tasks, and for durations up to at least $300 \mathrm{~ms}$; increased apparent duration for items at exogenously-cued locations is therefore a general result.

Insight into the origins of this effect comes from the separate observation that directed attention can impair temporal resolution. For visual stimuli, exogenously cuing a spatial location improves the detection of spatial gaps in stimuli presented at that location but hinders the ability to discriminate between two brief flashes and a single continuous presentation (Yeshurun \& Levy, 2003). Temporal order judgments are similarly impaired by this kind of exogenous attentional cuing (Hein, Rolke, \& Ulrich, 2006). Yeshurun and Levy attributed this impaired temporal processing to enhanced activity of the parvocellular neurons representing the attended location, with a corresponding decrease in magnocellular activity. Parvocellular neurons have smaller receptive fields (affording better spatial processing) but longer response latencies, resulting in less precise temporal coding. Consistent with this, detecting stimulus offset takes longer at cued locations (Rolke, Ulrich, \& Bausenhart, 2006). This neurophysiological mechanism may therefore explain the prolonged apparent duration of cued stimuli, but the specific argument is restricted to visual stimuli and exogenous cuing (endogenous cuing does not impair temporal resolution in the same way; Hein, Rolke, \& Ulrich, 2006); furthermore, some evidence suggests that the impaired temporal discrimination at cued locations is due to a speed-accuracy trade-off rather than to the sluggish activity of parvocellular neurons (Chica \& Christie, 2009).

Feature-based attending. Attention can be directed to particular stimulus features (for reviews, see Kanwisher \& Wojciulik, 2000; Maunsell \& True, 2006; Reynolds \& Chelazzi, 2004). Ono, Yamada, Chujo, and Kawahara (2007) have provided evidence that directing attention to particular stimulus features modulates subjective time. On each trial two stimuli (a circle-pattern and a squarepattern) were presented, with one stimulus type designated the 'target' for a given block of trials. Participants indicated the left-right location of the target; another stimulus then appeared centrally, 
and participants had to terminate its presentation after 2 seconds. Temporal productions were shorter (subjective time was longer) when this stimulus was the target from the first part of the trial than when it was the distractor. In other words, apparent duration was longer for stimuli whose features had recently been the focus of attention.

Time-based attending. Attention can also be directed at particular points in time, both intentionally and by the temporal structure of the task. Studies of endogenous temporal orienting present a symbolic cue at the start of the trial to signal when a target will appear (e.g., after a 'short' or 'long' delay). Target detection and discrimination are improved when the cue is valid (e.g., Correa, Lupiáñez, Milliken, \& Tudela, 2004; Coull \& Nobre, 1998; Miniussi, Wilding, Coull, \& Nobre, 1999), with corresponding enhancement of early evoked responses (Correa, Lupiáñez, Milliken, \& Tudela, 2004). Thus, people are able to focus processing resources at a particular moment, using a network of brain regions that partially overlaps with those activated by endogenous spatial orienting (Coull, Frith, Büchel, \& Nobre, 2000; Coull \& Nobre, 1998).

This endogenous temporal orienting also affects time perception (Correa, Sanabria, Spence, Tudela, \& Lupiáñez, 2006). Each trial began with a written cue indicating, with $75 \%$ validity, whether the target stimuli would appear 'early' or 'late' (i.e., after 400 or $1400 \mathrm{~ms}$ ). The targets were two LEDs attached to the sides of the monitor, and participants had to indicate which was turned on first. These temporal order judgments were more accurate when the orienting cue was valid, indicating that participants' endogenous temporal orienting had improved their temporal resolution. This study did not examine the effects of intentional temporal orienting on subjective duration and, as far as we know, no other study has done so; it would be helpful to establish whether orienting to a particular point in time expands apparent duration in the same way as endogenous spatial and modality orienting.

Temporal orienting can also arise exogenously by virtue of the temporal structure of sensory information (e.g., Jones, 1976, 2004; Jones \& Boltz, 1989; Large \& Jones, 1999). The most famous examples involve varying the interval between a neutral warning signal (e.g., a fixation cross) and the arrival of the target. When this foreperiod is manipulated between blocks of trials (a fixed foreperiod design), longer intervals result in worse performance, presumably because internal timing grows more variable with increasing duration (e.g., Mattes \& Ulrich, 1997). By contrast, when short and long foreperiods are intermixed in a variable foreperiod design, longer intervals lead to better performance, presumably because at the 'short' foreperiod the observer is uncertain about whether or not the stimulus will occur, but once that timepoint has been and gone they can be confident that the target will arrive at the later time (e.g., Grondin \& Rammsayer, 2003). The implicit temporal expectations generated by foreperiods have traditionally been assumed to affect motor preparation 
(Hackley, 2009; Müller-Gethmann, Ulrich, \& Rinkenauer, 2003), but it has become clear that they boost perceptual processing. For example, participants were faster and more accurate at detecting which side of a square contained a break when the fixed foreperiod was $800 \mathrm{~ms}$ than when it was 2400 ms (Rolke \& Hoffman, 2007). Similarly, tone frequency discrimination (Bausenhart, Rolke, \& Ulrich, 2007) and letter identification (Rolke, 2008) are better at short foreperiods, reflecting faster onset of evidence accumulation when stimulus arrival is more predictable (Bausenhart, Rolke, Seibold, \& Ulrich, 2010; Jepma, Wagenmakers, \& Nieuwenhuis, 2012; Seibold, Bausenhart, Rolke, \& Ulrich, 2011). Vangkilde, Coull, \& Bundesen (2012) provide recent evidence that these temporal expectations improve the quality of sensory information, and the speed of perceptual processing.

These changes in perceptual processing have corresponding effects on time perception. Grondin and Rammsayer (2003) had participants classify time intervals that varied around 500-ms as 'short' or 'long', with the foreperiod between their response and the next stimulus varying randomly from trial to trial. Longer foreperiods led to steeper psychometric functions and shifted the bisection point to the left, indicating improved temporal resolution and longer apparent duration for the more expected stimuli (recall that, when short and long foreperiods are intermixed, stimuli are more temporally predictable after long foreperiods). The effect of implicit orienting on subjective duration replicated for visually- and aurally-defined target intervals and across a range of foreperiods, generalized to shorter target durations (centred on $100 \mathrm{~ms}$ ), and has been replicated in other work (e.g., Gamache, Grondin, and Zakay, 2011; Los \& Horoufchin, 2011).

A separate line of work has concentrated on the temporal expectations generated by more complex event sequences. Of particular importance is dynamic attending theory (DAT; 1976, 2004; Jones \& Boltz, 1989; Large \& Jones, 1999), which describes how attention is guided in time by the predictable, hierarchical temporal/non-temporal structures of complex stimulus sequences (e.g., the rhythm and melody of music; 1976, 2004; Jones \& Boltz, 1989; Large \& Jones, 1999). In support of DAT, sequences which end earlier than expected are judged "short" whereas those that end late are judged "long" (Jones, Boltz, \& Klein, 1993), with corresponding effects for other types of timejudgment task such as tempo-assessment (e.g., Barnes \& Jones, 2000; Boltz, 1992, McAuley \& Kidd, 1998).

Importantly, the expectations generated by rhythmic structure also affect non-temporal processing; detection and discrimination are improved for stimuli whose onset fits with the rhythmic structure of preceding events than for items presented at unexpected times (e.g., Boltz, 1993; Cravo, Rohenkohl, Wyart, \& Nobre, 2013; Jones, Moynihan, MacKenzie, \& Puente, 2002; Miller, Carlson, \& McAuley, 2013; see Bauer, Jaeger, Thorne, Bendizen, \& Debener, 2015, for a recent null result, and Henry \& Herrmann, 2014, for a recent review). (Notably, these studies often find impaired 
performance for 'late' stimuli as well as 'early' ones, in contradistinction to the build-up of temporal expectations assumed in foreperiod studies). These changes in processing efficiency are again accompanied by expanded apparent duration: McAuley and Kim Fromboluti (2014) recently found that tones which occurred earlier than would be expected from a preceding rhythmic sequence were judged shorter than those that occurred on time, where those that arrived later than usual were judged longer.

Thus, implicit temporal orienting, like intentional orienting, improves perceptual clarity and information extraction, and expands the apparent duration of attended stimuli.

Attentional capture by significant stimuli. Attention is not just driven by predictive spatiotemporal cues: some stimuli are selected for preferential processing by virtue of their properties and significance. Indeed, we saw in Section 2 that salience is an over-arching principle behind the effects of basic non-temporal properties on time perception, and salient stimuli are preferentially processed and perceptually vivid by virtue of their attentional capture (see, for example, Eimer, Kiss, Press, \& Sauter, 2009; Itti, 2007; Itti \& Koch, 2001; Theeuwes, 2010). Stimuli can also capture attention by virtue of their biological significance rather than their novelty or distinctiveness. We describe two examples that illustrate how such effects influence subjective time.

Looming Motion. Franconceri and Simons (2003) reported that attention is automatically captured by stimuli that are moving towards the observer (looming). Participants searched among distractor letters to find which of two target letters was present. Each letter was hidden behind an individual mask; on some trials one of these masks started smaller than the others and expanded to be the same size as the rest immediately before the start of the visual search (looming motion); on other trials one mask started larger than the rest and shrank to their size (receding motion). When the target appeared at the location of the looming motion, response times were independent of the number of distractors, indicating attentional capture by this type of motion; this was not observed for the receding motion. Despite some controversy about this result (Abrams \& Christ, 2005, 2006; Franconeri \& Simons, 2005) the prioritization of looming stimuli has been found in other studies, and does not require motion onset (von Mühlenen \& Lleras, 2007; Skarratt, Cole, \& Gellatly, 2009).

The cuing effect of looming motion is mirrored in studies of subjective duration. van Wassenhove et al. (2008) found that smoothly-animated looming discs were judged to last longer than receding ones, and this effect transcended the relative frequencies of the two types of motion. Similarly, Ono and Kitazawa (2010) had participants judge the time interval between two circles presented in a display that included depth cues. The same interval was judged longer when the first circle was small and the second was large than when the order was reversed. That is, subjective duration was expanded for looming motion. The looming-receding difference has also been found 
for auditory and audio-visual stimuli (Grassi \& Darwin, 2006; Grassi \& Pavan, 2012, although the latter found no effect for purely visual stimuli).

Interesting, Ono and Kitazawa found no effect of looming motion on subjective time when the trajectory implied no observer-collision with the object, mirroring the finding that attentional capture is greater for objects on a collision path than for those with a near-miss trajectory (Lin, Franconeri, \& Enns, 2007). Taken together, the data suggest that the biological significance of a stimulus class can drive the allocation of processing resources, with a corresponding expansion of apparent duration.

Emotion. Emotional stimuli typically attract attention and are preferentially processed (see Yiend, 2010, for a review). Emotional items have lower detection thresholds (e.g., Calvo \& Esteves, 2005), are perceptually more vivid (e.g., Todd, Talmi, Schmitz, Susskind, \& Anderson, 2012), and are located faster during visual search (e.g., Öhman, Flykt, \& Esteves, 2001) than neutral ones, even after controlling for low-level stimulus features. When emotional and neutral stimuli are briefly presented side by side, attention is oriented to the emotional item such that detection and identification is superior for targets that subsequently appear at this location (e.g., Lipp \& Derakshan, 2005) - and disengagement from the location of emotional items takes longer than for neutral images (e.g., Fox, Russo, \& Dutton, 2002). Threatening stimuli (e.g., angry faces, snakes) are particularly likely to be preferentially processed, presumably because of their biological significance. Moreover, individual differences in background mood and clinical status moderate the effects of emotional stimuli (Yiend, 2010).

The enhanced processing of emotional stimuli is matched by an expansion in their subjective duration. Droit-Volet, Brunot, and Niedenthal (2004) provided a prototypical illustration. They used a bisection task in which the training phase established anchor durations of 400 and 1600 ms using a neutral shape, followed by a test phase in which photographs of neutral, sad, happy, and angry faces were displayed for intermediate durations that the participant classified as closer to the 'short' or 'long' standard. Relative to neutral faces the bisection point was shifted leftwards for all three emotional expressions, indicating longer subjective duration - with the overestimation particularly pronounced for angry faces.

This effect has been widely replicated with faces (e.g., Droit-Volet \& Gil, 2009), pictures (e.g., Angrillo et al., 1997; Grommet, Droit-Volet, Gil, Hemmes, Baker, \& Brown, 2010) and sounds (e.g., Mella, Conty, \& Pouthas, 2011; Noulhiane, Mella, Samson, Ragot, \& Pouthas, 2007), in children as young as 3 years (Gil, Niedenthal, \& Droit-Volet, 2007), and in cases where the emotional stimulus is presented below conscious awareness (Yamada \& Kawabe, 2011). These effects are not due to a low-level confound: the apparent duration of a neutral stimulus can be increased by conditioning an 
association to an emotionally-charged image (Kliegl, Watrin, \& Huckhauf, 2015) -- echoing the finding that threat conditioning can direct attention to previously-neutral images (Batty, Cave, \& Pauli, 2005).

Emotion effects on time perception do not reduce to simple differences in arousal (e.g., Angrilli et al., 1997; Gil \& Droit-Volet, 2012; Lake, LaBar, \& Meck, in press; Mella, Conty, \& Pouthas, 2011), and depend on the particular emotion, stimulus duration, and judgment task in ways that are not always consistent (e.g., Bar-Haim et al., 2010; Fayolle \& Droit-Volet, 2014; Gil \& Droit-Volet, 2011, 2012; Grommet, Droit-Volet, Gil, Hemmes, Baker, and Brown, 2010; Lee, Seelam, \& O’Brien, 2011). However, the prolonged apparent duration for threatening items is particularly robust, mirroring the strong evidence for superior processing of threatening stimuli in studies of visual attention. Moreover, just as the effects of threatening stimuli on attention are modulated by individual differences in mood and threat-sensitivity, so too is the expansive effect of threatening stimuli on subjective time. Tipples (2011), for example, found overestimation of duration for both fearful and threatening faces relative to neutral ones, and the effect was moderated by individual differences in fearfulness (see also Bar-Haim, Kerem, Lamy, \& Zakay, 2010; Tipples, 2008).

The complexity of emotion effects is unsurprising given the diversity of emotional stimuli and responses, but the data indicate that changes in perceptual clarity and processing that result from emotional relevance produce corresponding changes in apparent duration.

Selective attention and the processing principle. The allocation of attention may be based on the time, location, or sensory channel at which the stimulus is presented; it may be driven by the biological significance of the object; and it may be driven by exogenous spatio-temporal cues or endogenous shifts in focus. These shifts in attention can enhance processing of particular locations, modalities, or features. In all cases, the subjective duration of the attended object is increased. This unifying result provides further support for the processing principle: attending to a stimulus improves its subjective vividity and facilitates information processing, with a corresponding expansion in apparent duration. Attentional selection therefore complements the effects of stimulus properties discussed in Section 2. Indeed, to the extent that the effects of non-temporal features are due to their effects on salience, the line between 'external' physical properties and 'internal' allocation of attention is blurred: what matters is how the interplay of these factors shapes the overall clarity of the percept.

The powerful connection between attentional selection and subjective time suggests several lines for future research, including:

1) Generalizing to other types of attentional selection. Tactile and cross-modal cuing, for example, can enhance processing (e.g., Burton et al., 1999; McDonald, Green, Störmer, \& Hillyard, 
2012) and should therefore expand apparent duration. In addition, the time course of attentional shifts should produce complementary changes in subjective time. With exogenous cuing, processing at the cued location is actually impaired once the cue-target delay increases beyond a few hundred milliseconds (e.g., Posner \& Cohen, 1984). We predict that this inhibition of return (IOR) will produce a corresponding decrease in subjective duration at the cued location. Likewise, attention can be guided to particular locations by past experience with the same visual configurations ('contextual cuing'; Chun \& Jiang, 1998). Again, we would expect this implicit learning of context to boost the apparent duration of targets at anticipated locations. A key goal will be to integrate measures of perceptual clarity and subjective duration in the same experimental session, so that researchers can directly test the correspondence between the two.

2) Examining the effect of task relevance. Attentional selection depends on the organism's current goals and disposition (e.g., Bacon \& Egeth, 1994). Do task demands also modulate the apparent duration of otherwise identical stimuli? As for the effects of emotional material, individual differences might be important: the apparent duration of a monetary stimulus, for example, may depend on the participant's acquisitiveness.

3) Clarifying the mechanisms by which selective attention affects the sense of time. As noted, improved onset detection and delayed offset detection may be contributing factors but are not the whole story (e.g., Enns et al., 1999; Rolke et al., 2006); likewise studies with emotional stimuli suggest a slope effect, implying an increase in the rate of growth of subjective time (not just a shorter onset-detection latency, which would produce a fixed effect that is independent of the subsequent duration of the item) -- at least for intervals up to about 1 second (e.g., Gil \& Droit-Volet, 2012). More generally, however, we need to examine the effects of selective attention across a wide range of durations and to measure onset- and offset-detection latencies to quantify the separate processes underlying changes in time perception.

\section{Divided Attention}

Moving from selective attention to stimuli to the distribution of processing resources across tasks, a large body of work has examined how temporal judgments are affected by the activities that are performed during the timed interval. This research has a long tradition (e.g., Yerkes \& Urban, 1906) and has often focussed on situations where participants perform a task such as card-sorting for several minutes before estimating how long they spent on it (e.g., Hicks, Miller, \& Kinsbourne, 1976). We focus on work which has used shorter durations akin to those discussed elsewhere in this review. 
Doing two things at once: Dual-task effects on time judgments. Temporal judgments are influenced by a wide variety of secondary tasks. In one influential study, S.W. Brown (1997) had participants make a series of temporal productions by pressing a key every 2 or 5 seconds at a steady rate throughout a two minute trial, while the participant simultaneously undertook a separate cognitive task: pursuit rotor tracking (manually following a visual target); visual search (scanning a page and marking with a pen each occurrence of a target letter); and mental arithmetic (subtraction problems); all three tasks came in 'easy' and 'hard' versions. In baseline trials, participants completed either the time production or cognitive tasks in isolation. Compared to baseline performance, undertaking a secondary task disrupted timing: temporal productions became more variable and, in general, longer, and these effects became more pronounced as the difficulty of the secondary task increased.

These findings have been replicated using a wide range of different secondary tasks (see S.W. Brown, 2008, 2010, for reviews). In a recent comprehensive meta-analysis of 117 experiments, Block, Hancock, and Zakay (2010) found that prospective duration judgments were systematically shorter and more variable with higher cognitive load. The effects were strongest for production tasks like those used by Brown (1997), but generalize to verbal estimation and reproduction. Interestingly, for retrospective duration estimates (where the participant does not know that a time judgment will be required until after the interval), cognitive load lengthens subjective duration, suggesting a memory-based mechanism in which judged duration is proportional to the number of segments or changes in the retrieved interval (Block et al., 2010).

The effects of secondary tasks on prospective time estimates are typically explained in terms of attentional allocation: People may direct limited processing capacity towards either 'temporal' or 'non-temporal' information (Brown, 2008; Buhusi \& Meck, 2009; Michon, 1972; Thomas \& Weaver, 1975), and directing attention away from time results in less accrual of temporal information and ergo shorter and more variable temporal estimates. 'Temporal information' risks being ill-defined, but one conceptualization posits an attentional 'gate' that controls the flow of pulses from a pacemaker into an accumulator during the interval (Zakay \& Block, 1997); another suggestion is that attention determines the latency with which the flow of pulses begins at stimulus onset (Lejeune, 1998; Meck, 1984) (see Figure 4 panel A).

The allocation of mental resources to timing is under intentional control. In one demonstration, Macar, Grondin, \& Casini (1994) presented sequences of words and had people count the number of animal names and then reproduce the duration of the whole list under instructions to allocate $0,25,50,75$, or $100 \%$ or their attention to the temporal task (with the remainder dedicated to the non-temporal task). Temporal reproductions shortened as more 
attention was devoted to the animal-counting task, again suggesting that subjective duration was reduced when participants were not 'attending to time' (see also Franssen and Vandierendonck, 2002; Zakay, 1998). Likewise, when participants judged both the duration and luminance of a light, instructions to devote greater attention to the luminance task resulted in poorer temporal discrimination and shorter subjective duration (Casini \& Macar, 1997).

Attention and anticipation. As well as comparing time judgments with and without a secondary task, researchers have examined the effects of varying the time at which a secondary event occurs during a to-be-timed interval. In Casini and Macar (1997), participants studied a green light whose intensity briefly increased by either a small or large amount during the course of its presentation. After offset, they indicated both the duration of the light and the size of the intensity change. When the intensity increment occurred later in the presentation, the subjective duration of the light was reduced. This location effect has been taken to indicate that participants devote some attention to monitoring for the intensity change, diverting resources away from the accrual of temporal information: the later the intensity change occurs, the longer this monitoring goes on and the shorter the subjective duration. The location effect has been widely replicated (e.g., Fortin, 1999; Rousseau, Picard, \& Pitre, 1984), and there is some evidence that anticipation of a secondary signal may produce the effect even when there is no requirement to process it, although support for this is mixed (Champagne \& Fortin, 2008; Gaudreault, Fortin, \& Macar, 2010; Macar, 2002).

In fact, anticipation effects can disrupt timing without employing a secondary task at all, by inserting a break in the stimulus presentation. An early example is provided by Fortin and Masse (2000). On each trial, participants pressed a key to start presentation of a tone and made a second key press to terminate the sound. After learning to produce 2 -second intervals, participants undertook a test phase in which the tone was interrupted at various points by silent breaks of between 3 and 6 seconds, with the task still being to make the total duration of the tone (excluding the gap) 2 seconds. The key finding was that productions were shorter when the break came $500 \mathrm{~ms}$ into the tone presentation than when it came after $1500 \mathrm{~ms}$. Just as for dual-task studies, Fortin and Masse attributed this location effect to attention-sharing: before the break, participants are monitoring for the interruption, which diverts processing away from temporal information. The later that the break occurs, the more time information is lost and the shorter the subjective duration of the pre-break segment - with the result that it takes longer for the total judged duration of the tone to reach 2 seconds.

Consistent with this, subsequent experiments showed that the temporal productions were even longer for 'no break' trials, where the participant waited for an interruption throughout the whole of the tone presentation; moreover, signalling that there will be no break at the start of the 
trial greatly reduced this effect, implying that when people are no longer monitoring for the interruption of the tone they can direct more attention to time and accrue subjective duration more rapidly. The effects of break expectancy have been replicated in other studies using different timeestimation procedures (e.g., Fortin et al., 2009; Fortin \& Tremblay, 2006; Tremblay \& Fortin, 2003) and are widely found in animals (e.g., Buhusi and Meck, 2009).

An 'executive gate'? A straightforward attention-allocation model implies a common pool of resources used by both the temporal and non-temporal tasks. This, in turn, predicts bidirectional interference: devoting more attention to time should lower performance on a secondary task (S.W. Brown, 1997). However, those studies that have examined performance on the non-temporal task have not found universal two-way interference. In S.W. Brown's (1997) experiment, for example, temporal production impaired mental arithmetic but not motor pursuit or visual search. In a survey of 33 studies, S.W. Brown (2006) noted that bidirectional interference was present for tasks such as mental arithmetic, proof-reading, or searching working memory, but that activities such as motor tracking or visual search were unaffected by concurrent timing. Bidirectional interference seems to be most pronounced for tasks that tap the putative 'central executive' component of working memory (Block et al., 2010; S.W. Brown, 2008), such as random-number generation and sequential reasoning (S.W. Brown, 2006).

This has led to the proposal that, rather than an undifferentiated processing capacity, 'attending to time' specifically uses executive control processes - those which "co-ordinate working memory subsystems, focus and switch attention, and activate representations within long-term memory" (Fortin, Schweickert, Gaudreault, \& Viau-Quesnel, 2010, p. 580). Within the pacemakeraccumulator framework, Block et al. (2010) suggest replacing the putative attentional gate with an 'executive gate'. The close links between executive processing and timing make sense given the temporal aspects of many executive functions (sequential processing, scheduling, etc.) and the shared neural substrates of timing and executive processing (S.W. Brown, 2008).

Despite the appeal of this suggestion, it has a number of shortcomings. First, despite taskswitching being a prototypical executive function, recent work by Fortin et al. (2010) found that it did not disrupt timing (although S.W. Brown, Collier, \& Night, 2013, reported a conflicting result). More importantly, the executive-function account does not explain why timing is disrupted by many tasks which make very little use of executive control, such as gauging the size of a luminance increment or monitoring for a break in the presentation of a tone. Indeed, as Brown et al. (2013) recently noted, "virtually any type of distractor task interferes with temporal judgments" (p. 947). One methodological problem here is that researchers have not equated the difficulty of the temporal and non-temporal tasks. It may be that the unidirectional interference arises because the 
timing tasks are simply more difficult than the secondary tasks and therefore more vulnerable to interference. Using perceptual discrimination as a secondary activity, it ought to be possible to ensure that temporal and non-temporal judgments are matched for difficulty.

Divided attention and the processing principle. The processing principle is the generalization that the subjective duration of a stimulus is positively related to its perceptual clarity. As such, it does not directly speak to the effects of dividing attention between judging time and judging other stimulus properties or performing secondary tasks. Indeed, in many dual-task studies there is no one stimulus whose duration is to be judged; rather, participants are confronted with multiple, overlapping stimuli and self-generated actions (e.g., trying to produce a button press every 3 seconds whilst searching a sheet of paper for every occurrence of the letter ' $T$ '). The processing principle does not readily extend to such complex situations. Even in the simpler 'anticipation' tasks, it is unclear how interrupting a stimulus with a secondary event or gap (or how anticipating these events) would be expected to influence the perceptual vividity of the pre- and post-interruption segments; and whatever the effects on each segment, the overall duration judgment will depend on integrating separate representations in ways which are not currently well-understood (Bryce \& Bratzke, 2015; Matthews, 2013).

The processing principle is therefore best regarded as orthogonal to the resource allocation processes that underlie dual-task performance: the representation of stimulus duration conforms to the processing principle, but how much mental capacity is devoted to constructing this representation, and how much weight this information receives, depend on the organism's goals and competing task-demands. Nonetheless, an important aim for the future will be to establish how the totality of perceptual experience shapes subjective time - that is, how people integrate information from multiple, temporally overlapping stimuli, with varying perceptual strengths, to form an overall sense of time.

\section{The relevance of subjective time to attention researchers}

The links between attention and subjective time carries several implications and opportunities for attention researchers.

First, subjective duration provides an aspect of human experience to which attention researchers can apply their existing theories and methodologies, testing generality and, potentially, finding results that challenge current thinking. As noted above, questions such as whether inhibition of return affects the subjective duration of targets at cued/uncued locations provide straightforward empirical opportunities. Similarly, researchers who develop and use theoretical models to predict the allocation of visual or auditory attention (e.g., Itti \& Koch, 2001; Oldoni et al., 2013) could see 
whether their accounts successfully account for the apparent duration of targets appearing at particular locations - perhaps refining their models to yield precise quantitative predictions. Relatedly, attention researchers might consider using subjective duration as an index of attention to a stimulus - a sign that it is being preferentially processed by virtue of its basic properties, spatiotemporal position, or goal-relevance, which could be explored more deeply by subsequent studies of discriminability, subjective salience, memory encoding, and so forth.

Second, there has been growing appreciation that spatial and temporal allocation of attention may share common mechanisms and neural substrates (Coull \& Nobre, 1998; Rohenkohl, Gould, Pessoa, \& Nobre, 2014); the finding that spatial and temporal orienting exert comparable effects on the phenomenology of subjective time adds weight to this argument and provides impetus to the quest for integrated accounts. It also raises the question of whether attention allocation might take place in subjective time. We have seen that temporal expectations can sharpen the representation of stimuli that arrive 'when expected'. The processing principle implies that these expectations will depend on the perceptual strength of the stimuli that define the to-be-timed interval. As a simple example: if the same foreperiod is filled with a low intensity tone, it will seem shorter than if it is filled with a high intensity tone. These changes in subjective time will presumably affect whether a subsequent target seems to arrive late, early, or on time, with corresponding changes in detection and discrimination accuracy.

Finally, we have noted that the processing principle currently applies to relatively simple situations where people judge a single stimulus. How are the representations of multiple, temporally-overlapping items combined to produce an overall sense of time for complex, on-going patterns of multi-modal experience? This major theoretical challenge is one that attention and multi-tasking researchers are well-placed to help solve.

\section{Section Summary}

Attention profoundly affects time perception. Directing attention to a stimulus facilitates processing and prolongs apparent duration; directing mental resources towards a temporal judgment task similarly lengthens subjective time. We have suggested that these effects can usefully be construed as part of a general information-processing framework, which could serve as a starting point for future theoretical and empirical work. The foregoing research also highlights the key role of working memory in timing, a point that we explore further in the next section.

\section{Section 4: Subjective Time and Memory}


Memory is central to temporal perception and judgment. As noted above retrospective judgments are thought to be based on memory for the number of changes that took place during the interval (Block et al., 2010; Poynter, 1983). In the prospective tasks that are the focus of this review, time judgments depend on the comparison of the target duration with previously-encoded intervals (Matell \& Meck, 2004; Wearden, 1992). Moreover, the accuracy and precision of temporal representations changes as a function of experience both with the target duration (e.g., Bueti \& Buonomano, 2014; Kristofferson, 1980; MacDonald, Cheng, \& Meck, 2012; Matthews \& Grondin, 2012; cf., Jones and Wearden, 2003) and with other recently-encountered intervals (e.g., G. D. A. Brown et al., 2005; Dyjas, Bausenhart, \& Ulrich, 2012; Hellström, 2003). Recent work has explored the effects of aging (Droit-Volet, 2003; Droit-Volet \& Zélanti, 2013; Gooch, Stern, \& Rakitin, 2009) and emotion (Cocenas-Silva, Bueno, \& Droit-Volet, 2013) on memory for time, has examined whether a consolidation process occurs for duration memories similar to the synaptic/cellular mechanisms observed for other types of memory (Cocenas-Silva, Bueno, \& Droit-Volet, 2014), and has formally modelled the formation and interference of temporal memories within the framework of internal clock models (L.A. Jones \& Wearden, 2003; Ogden, Wearden, \& Jones, 2008).

Here, we focus on memory as it relates to the non-temporal aspects of the stimulus - that is, on how the subjective duration of an item changes as a function of its previous encoding and storage. Following the 'textbook' organization, we first consider low-level sensory adaptation to stimulus features and then progress to representations of items encountered in the past few seconds ('short term' memory), which may be maintained and manipulated for an on-going task ('working memory'), and end with the effects of memory for items encoded several minutes, hours, or even days before ('long term' memory). Like many other authors, we use this as a convenient way to organize the research findings, with no theoretical commitment to distinct neural structures or processing systems.

\section{Sensory adaptation}

We begin by considering recent work on the effects of low-level adaptation to stimulus features. Although this type of prior-exposure effect might typically be thought of as 'sensory' rather than 'mnemonic' (indeed, some of the following material could have appeared in Section 2 of this paper), we include it here because, like other 'memory' effects, it concerns the effects of previous encounters with/encoding of the stimulus on the perception of time..

Adaptation is the change in processing that follows exposure to a constant sensory input, such as when one ceases to feel of one's clothes within a few seconds of putting them on. The effect underlies visual illusions in which unchanging peripheral stimuli seem to fade away when one 
maintains constant fixation (Martinez-Conde, Macknik, \& Hubel, 2004), and also occurs with higherlevel representations, as illustrated by face aftereffects (e.g., Leopold, O’Toole, Vetter, \& Blanz, 2001). The reduced perceptual strength that accompanies sustained, steady stimulation is based on a 'recalibration' of the sensory system to become more sensitive around the adapted level (see e.g., Clifford, 2002; Thompson \& Burr, 2009), and this occurs for high-level representations as well as lowlevel features (e.g., Rhodes, Watson, Jeffery, \& Clifford, 2010).

Interest in the role of adaptation in time perception was recently sparked by Johnston, Arnold, and Nishida (2006). They presented a drifting sine grating to the left or right of a central fixation point. The grating had a temporal frequency of $20 \mathrm{~Hz}$ and alternated direction every 2 seconds to prevent motion aftereffects. After adapting to the grating for 15 seconds participants were sequentially presented with two gratings drifting at $10-\mathrm{Hz}$ and judged which had longer duration: a 600-ms standard, presented on the adapted side, or a variable-duration comparison stimulus, presented on the unadapted side. There was substantial duration compression in the adapted location: the standard seemed to last less time than an equivalent stimulus in the unadapted location. This effect was not due to onset- or offset-detection latencies, and also occurred with simple luminance flicker rather than spatial drift.

Adaptation a particular stimulus feature can therefore produce a spatially-specific reduction in the subjective duration of subsequent stimuli at that location. Johnston and colleagues have argued that this effect is retinotopic and sub-cortical: it occurs with narrow adapting stimuli (consistent with the small receptive fields of early visual neurons; Ayhan, Bruno, Nishida, \& Johnston, 2009) and when the orientations of the adaptor and target stimuli differ by 90 degrees (orientation sensitivity only emerges in cortical area V1; Johnston et al., 2006). On the other hand, Burr, Tozzi, and Morrone (2007) shifted the fixation point after adaptation such that the standard stimulus occupied either the retinotopic or spatiotopic position of the adaptor, or a completely new position. Only the spatiotopic condition compressed subjective duration, indicating a cortical locus for the adaptation effect (see Bruno, Ayhan, \& Johnston, 2010; Burr, Cicchini, Arrighi, \& Morrone, 2011; Morrone, Cicchini, \& Burr, 2010 for further discussion). Recent work suggests effects at multiple levels in the processing hierarchy (Bruno, Ng, \& Johnston, 2013; Latimer, Curran, \& Benton, 2014).

Importantly, adaptation effects have been found for other stimulus features, with shorter adaptation periods, and in different modalities. Curran and Benton (2012) adapted participants to an upward-drifting dot pattern and found compressed duration for subsequent stimuli in the adapted location, but only when they drifted in the same direction as the adaptor. This difference was not due to changes in the time taken to detect stimulus onset or offset. Elsewhere, Bruno and Johnston 
(2010) found an adaptation effect for luminance contrast using prior exposures of just 1.5 seconds, and Watanabe, Amemiya, Nishida, and Johnston (2010) found shortened subjective duration for vibrotactile stimuli presented at locations which had adapted to higher-frequency vibrations. One particularly powerful demonstration was recently provided by Zhou et al. (2014), who had participants compare two identical Gabor patches presented to the left and right of fixation. The first, standard stimulus was preceded by a 107-ms presentation of another Gabor patch, identical to the other stimuli except for its orientation. This prime compressed the apparent duration of the standard, and the size of this compression diminished as the orientation difference between the prime and standard increased.

Thus, as a general principle, recent exposure to basic stimulus properties leads to localized reductions in perceptual vividity and the compression of subjective duration for subsequent stimuli sharing those features. The effects of adaptation therefore provide a further illustration of the processing principle: adaptation reduces the effective strength of the sensory input, weakening the final percept and shortening subjective duration. This consequence of prior exposure helps to shed light on the complex effects of stimulus repetition discussed next.

\section{Sensory and Short-term memory}

Moving 'up' from studies of basic stimulus properties to the encoding of whole objects, many studies have examined how recent exposure to a stimulus influences its apparent duration. Much of this research has used a so-called 'oddball' paradigm pioneered by Tse et al. (2004) in which participants see or hear a standard stimulus several times in succession and a different stimulus is displayed somewhere towards the end of the sequence. For example, Tse et al. presented a static black disc for 1050 ms, separated by inter-stimulus intervals (ISIs) of 950-1150 ms. After every 7-12 occurrences of the standard, an expanding black disc was presented for a variable duration and participants indicated whether it was shown for more or less time than the standards. The point of subjective equality indicated that the oddball only had to be on-screen for $675 \mathrm{~ms}$ to 'feel as long' as the 1050-ms standards .

Tse et al.'s (2004) studies exaggerated the size of the novelty effect by using comparison durations that tended to be shorter than the standard, but the oddball effect remains robust when a symmetric distribution is used (Seifried \& Ulrich, 2010) and has been replicated many times (e.g., New \& Scholl, 2009; Pariyadath and Eagleman, 2007, 2012; Schindel, Rowlands, \& Arnold, 2011). The effect arises in both visual and auditory modalities (e.g., Kim \& McAuley, 2013; Tse et al., 2004), and generalizes to other judgment tasks such as magnitude estimation and equality judgments 
(Birngruber, Shroeter, \& Ulrich, 2014; Tse et al., 2004) which has been taken to indicate a genuine perceptual distortion rather than a decision bias.

The oddball task confounds stimulus novelty with position in the sequence (oddballs occur later in the stream) and involves comparing the single oddball's duration against multiple standards. However, the repetition effect replicates when there are just two stimuli on each trial and the second is either a repetition of the first or a novel item (Matthews, 2011c). In addition, the first presentation in a train of repeated stimuli seems longer than subsequent occurrences (Rose \& Summers, 1995).

The repetition effect reported in these studies complements the low-level adaptation effects discussed above, but the two sets of findings have typically been treated separately, and researchers have offered several overlapping explanations for the oddball/immediate repetition effects described here.

Attention to the non-repeated item. Tse et al. (2004) attributed the repetition effect to greater attention to novel stimuli, arguing that rare items "trigger[s] an increase in perceptual information processing" (Tse et al., 2004, p. 1187). This echoes our processing principle, and is supported by evidence that novel or unexpected stimuli commonly draw processing resources (e.g., Horstmann, 2002; Irwin, Colcombe, Kramer, \& Hahn, 2000; Meyer Niepel Rudolph Schützwohl 1991). A potential objection is that repeating an item can generate an expectation or 'attentional set' that improves its perceptual clarity, which in turn would make repeated items seem to last longer than novel ones (Ulrich, Nitschke, \& Rammsayer, 2006) - an idea to which we return below. More generally, studies of repetition effects on subjective time have not assessed the processing of non-temporal features, and have positioned the novel and repeated items in the same spatial location. By independently assessing whether processing is enhanced for novel stimuli, and whether attention is drawn to their position, researchers could directly test the attention-capture hypothesis.

Arousal. Ulrich et al. (2006) proposed that rare items are more arousing than repeated ones, leading to a brief elevation in the rate of an internal pacemaker. The repetition effect only emerges for stimuli longer than about $300 \mathrm{~ms}$, consistent with it taking some time for the pacemaker to 'ramp up' in response to the novel item (Seifried \& Ulrich, 2010). In addition, New and Scholl (2009) found that the apparent duration of a central square was expanded when a peripheral oddball occurs during its presentation, which they took as evidence for a generalized increase in arousal rather than a localized effect of directed attention to the novel stimulus. However, Pariyadath and Eagleman (2007) found no evidence that a visual oddball altered the apparent pitch of an accompanying tone which would be expected if there were a truly global change in temporal processing -- and van 
Wassenhove et al. (2008) similarly found no effect of an auditory oddball on the judged duration of an accompanying visual stimulus.

Predictive coding. Repetition effects are central to Eagleman and Pariyadath's (2009) coding efficiency account of time perception, described in Section 2 (Matthews et al., 2014). Recent exposure reduces the neural response to a repeated item. One view is that this repetition suppression is due to low-level processes such as neural fatigue; another is that it reflects predictive coding (De Baene \& Vogels, 2010; Grill-Spector, Henson, \& Martin, 2006; Henson \& Rugg, 2003). Predictive coding is the wide-ranging idea that the brain predicts sensory input, rather than simply processing incoming information 'bottom up'. A common proposal is that back-projections from later stages in the processing hierarchy signal expectations, such that only information that deviates from predicted inputs ('prediction error') is passed on to the next step in the representational hierarchy (e.g., Friston, 2005; Rao \& Ballard, 1999; see Clark, 2013, and the associated commentaries for a recent in-depth review).

Pariyadath and Eagleman $(2007,2008,2012)$ attribute the reduced subjective duration of repeated items to repetition suppression, and favour a predictive-coding explanation. In keeping with this, the more times a repeated standard is presented in succession, and the greater the deviation between the oddball and the standard, the greater the relative subjective duration of a subsequent oddball (Kim \& McAuley, 2013; Pariyadath \& Eagleman, 2012.

Moreover the subjective durations of novel and repeated stimuli are correlated with the strength, rather than the timing, of neural activity in high-level visual areas (Noguchi \& Kakigi, 2006; see also Sadeghi, Pariyadath, Apte, Eagleman, \& Cook, 2011), and the judged duration of oddballs increases with their discrepancy from the standard (i.e., with the mis-match between the 'predicted' and 'observed' stimuli; Pariyadath \& Eagleman, 2012; Schindel, Rowlands, \& Arnold, 2008) providing further support for the predictive-coding account [see also Zhou et al.'s (2014) study with Gabor patches, above].

Repetition and expectation. The attention, arousal, and predictive-coding accounts share the assumption that it is the predictability of repeated items that compresses their apparent duration: items which have not previously (or recently) been seen are more captivating, arousing, and unpredictable than those which are held in short-term memory. The idea that stimulus repetition shapes implicit expectations is widespread in perception and memory research (e.g., Bodner \& Masson, 2001) and is central to the predictive coding account of neural repetition suppression (e.g., Friston, 2005).

A clear theoretical problem, however, is that the studies of attention reviewed above clearly demonstrate that making an item more predictable - as happens with repeated stimuli - tends to 
increase rather than decrease its apparent duration. There is also an empirical problem, in that studies which only manipulate the memory-status of an item do not disambiguate the contribution of predictability and expectation from that of 'mere' repetition per se.

However, mere-repetition and predictability effects can be separated by presenting two stimuli on each trial and varying the proportion of trials on which the second is a repeat of the first. If repetition effects are due to the mere recent encoding of the item, then they will depend only on whether the second stimulus in the pair is the same as the first. However, if the effect also depends on implicit expectations about forthcoming stimuli, then making repetition trials more common should enhance the expectation that the second image will be a repeat and correspondingly increase the size of the repetition effect.

Neuroimaging studies using this approach have found that repetition suppression is more pronounced when repeat trials are common, suggesting that the reduced neural response reflects implicit expectations about the probability that the recently-encoded item will be encountered again (Summerfield, Trittschuh, Monti, Mesulam, \& Egner, 2008; see also Mayrhauser, Bergmann, Crone, \& Kronbichler, 2014; Summerfield, Trittschuh, Monti, Mesulam, \& Egner, 2008; Summerfield, Wyart, Johnen, \& de Gardelle, 2011), although a minority of studies have found that the repetition effect is independent of repetition probability (Kaliukhovich \& Vogels, 2011; Kovács, Kaiser, Kaliukhovich, Vidnyanánszky, \& Vogels, 2013). A similar pattern is found in many behavioural tasks. For example, in studies of masked priming, increasing the proportion of trials on which the target is a repeat of the prime leads to stronger facilitation of number judgments, lexical decision, and word naming, implying that implicit expectations lead to the recruitment of recently-encoded stimuli to facilitate subsequent processing (Bodner \& Dypvik, 2005; Bodner \& Masson, 2001, 2004).

Matthews (2015) recently applied this approach to temporal judgment. Participants saw two images on each trial and judged whether the second had longer or shorter duration than the first. Trials differed in whether the second image was a repeat of the first or a novel picture, and the proportion of 'repeat' trials varied between blocks. If short-term memory for a stimulus reduces its apparent duration by making it more expected, then the repetition effect should be more pronounced when repeats are common (the pattern seen in the neuroimaging data). However, making repetitions more probable reduced, eliminated, and even reversed the usual compression of subjective duration for recently-studied items. This argues against the expectation-based attention, arousal, and predictive-coding accounts described above; it is also incompatible with a simple 'mererepetition' effect, which predicts no effect of changing the repetition rate.

Rather, the data suggest that short-term encoding of a stimulus produces two opposing effects: a mere-repetition effect that shortens apparent duration, and an expectancy effect that prolongs it. 
The mere-repetition effect is analogous to the low-level adaptation effects described in the previous section, where we saw that recent, even quite brief prior exposure to a given stimulus feature can produce a spatially-specific compression for subsequent stimuli that share the adapted features. Countering this effect, the increased predictability of repeated items when repeats are common means that the first image in a pair would be a good cue to the features of the second and, analogous to the cuing paradigms discussed above, would be expected to improve the perceptual strength of the stimulus and expand its apparent duration.

Consistent with this framework, Matthews (2015) found that increasing the interval between the first and second presentations of a given image to 2 seconds eliminated the usual compression of subjective duration, mirroring the rapid perceptual and neural recovery from brief adaptation (Glasser, Tsui, Pack, \& Tadin, 2011) and suggesting a low-level process such as neural fatigue (De Baene \& Vogels, 2010). In addition, changing the predictability of repeats affected performance when participants had to classify the gender of the images rather than judge their durations, with the data showing that the increased apparent duration of predictable items was matched by improved efficiency of information extraction.

Immediate repetition and the processing principle. Recent exposure therefore has multiple effects on apparent duration. On the one hand, repetition reduces the effective strength of the sensory input via adaptation, and novel items are likely to be preferentially selected for processing because of their attention-grabbing salience. On the other, recent presentation is posited to generate an expectation that the same item will occur again, with a corresponding boost to perceptual clarity. The rather confusing pattern of data from the foregoing studies can therefore be understood in terms of the interplay between 'bottom up' and 'top down' factors posited by the processing principle; the same applies when we consider the active maintenance of recentlyencoded information in working memory.

\section{Working memory}

Maintaining a stimulus or feature in working memory modulates temporal judgments for stimuli with that property. Pan and Luo (2012) showed participants a square (the 'cue') followed by two coloured circles, one after the other, with the requirement to indicate which circle had the longer duration. One of the circles was the same colour as the cue; the other was different. Finally, participants saw a test stimulus and indicated whether it matched the cue - so participants had to hold the cue item in memory throughout the temporal judgement task. The probability of judging a test circle as lasting longer was elevated when its colour matched the item in working memory: 
actively maintaining a stimulus representation in working memory expanded the judged duration of stimuli with matching features.

This occurred even when the cue stimulus was replaced by a written colour name; maintaining this semantic information in working memory was sufficient to increase the judged duration of subsequent stimuli possessing that feature. The importance of active maintenance was demonstrated by a condition in which there was no requirement to remember the colour of the initial square: now the circle whose colour matched this cue was judged to have shorter duration, akin to the immediate repetition effect found in oddball tasks discussed above.

Active maintenance may over-ride the effects of basic properties or salience. Bi, Liu, Yuan, and Huang (2014) had participants memorize a digit at the start of the trial and then compare the duration of two other digits. When neither of the digits in the comparison task matched the item in memory, the authors found the usual association between larger numbers and longer time judgments. However, this disappeared when one of the digits matched the item in working memory, suggesting that active maintenance over-rides any association between temporal and non-temporal magnitudes (e.g., Walsh, 2003), or salience-based expansion for larger numbers. The importance of active maintenance was again emphasized by the finding that no such modulation of the magnitude effect occurred when the requirement to memorize the initial digit was lifted.

These results are reminiscent of Ono et al.'s (2007) study, described in Section 2, in which stimuli whose features had recently been searched for in a localization task had longer subjective duration than those whose features had recently been ignored. Taken together the results suggest that judged duration is longer for stimuli whose feature-representations are active when the stimulus is presented.

These time-perception data accord with a broader body of work examining the neural and behavioural effects of maintaining a stimulus representation in working memory. For example, Soto, Humphreys, and Rotshtein (2007) presented a shape cue (e.g., a red square) after which participants indicated which of two shapes contained a target stimulus. When the target appeared in the cued shape, responses were faster than then it appeared in an uncued shape, but only when participants were required to hold the cue in working memory throughout the trial. These behavioural effects have neural analogues: mere repetition of the cue decreased activity in a network including the parahippocampal gyrus, lingual gyrus, and superior frontal gyrus - akin to the repetition-suppression discussed above -- but when the cue was actively maintained its re-occurrence caused increased activity in these areas

These studies all fit with the idea that judged duration is positively related to the accessibility and clarity of perceptual representations - that is, with the processing principle. 
Maintaining feature-representations in an active state, ready for a subsequent memory test, means that stimuli containing those features are processed more rapidly and accurately (with a corresponding enhancement of neural activity) (Soto et al., 2007), and these stimuli are judged to last longer (Pan \& Luo, 2012). As yet, researchers have not integrated these effects in a single paradigm. It would be productive to adapt the procedure of Soto et al. (2007) to include a temporal judgment task and to see whether the improved processing and enhanced neural responding for features in working memory directly map on to increased subjective duration.

\section{Long-term memory}

Encoding a stimulus into long-term memory typically expands its apparent duration when it is re-presented. This was demonstrated by Witherspoon and Allan (1985), who presented participants with a list of 80 words for $1 \mathrm{~s}$ per word. When participants later classified the durations of words presented for 30 or $50 \mathrm{~ms}$ each, words from the studied list were judged to last longer than novel ones. Masson and Caldwell (1998) found the same effect using a similar design, but where participants generated the studied words from semantic cues, and Paller, Mayes, McDermott, Pickering, \& Meudell (1991) found the effect in amnesic patients.

Witherspoon and Allan (1985) attributed their data to a version of the processing principle, suggesting that studied items are easier to process and seem more familiar, and that this ease-ofprocessing is interpreted by the participant as evidence that the item must have been presented for a longer duration -- in the same way that making an item easier to read can lead to the belief that it has been recently studied (e.g., Whittlesea, 1993). Consistent with this, when Witherspoon and Allan had participants identify the test words immediately before making their duration judgments, the studied words were better identified as well as seeming to last longer. The improved processing of recent items can, like the expectation-effects discussed above, be construed in terms of the predictability of forthcoming stimuli: in the environment, recently-encountered items are likely to occur again in the immediate future, and the dynamics of long-term memory retrieval are tuned to this statistical structure (Anderson and Schooler, 1991).

These long-term memory effects have been generalized to longer durations and alternative tasks. Ono and Kawahara (2008) had participants study words and then presented a mix of studied items, novel items, and 'critical lures' which were semantically associated with the studied words. Participants had to press a button to terminate presentation after each test word had been displayed for $2.5 \mathrm{~s}$. Temporal productions were shorter for studied than for novel items, implying longer subjective duration, and this effect generalized to the critical lures, suggesting that conceptual processing underlies the memory effect. 
An exception the usual pattern comes from Ono and Kawahara (2005), who repeated dot patterns across trials and found that participants did not recognize the repeated displays, but that these stimuli had shorter apparent duration than novel ones - akin to the repetition effects in oddball tasks and studies of low-level adaptation. This might indicate that conscious recognition is necessary for the expansive effects of long-term memory, but unfortunately this study used a production methodology, so the shorter productions for familiar patterns might simply indicate that participants became bored of looking at them more rapidly (Matthews, 2011c). Nonetheless, the expansive effect for recognizable stimuli, and the compressive effect for unrecognized repeats, echoes the difference between active maintenance and passive exposure seen in studies of working memory.

Finally, pre-existing long-term representations also expand subjective duration. Familiar, high-frequency words presented for $1 \mathrm{~s}$ are judged longer than low-frequency words (Warm, Greenberg, \& Dube, 1964; Warm \& McCray, 1969; see also Devane, 1974), and briefly-presented words are judged longer than non-words (e.g., Reingold \& Merikle, 1988; Reber, Zimmermann, \& Wurtz, 2004) - although these effects are sometimes fragile (see Reber et al., 2004 for discussion). Again, these findings support the generalization that conditions which facilitate non-temporal perceptual processing and information-extraction also increase duration judgments.

These long-term memory effects suggest the straightforward prediction is that variables which affect memory retrieval (e.g., recency, interference, depth of encoding) will produce corresponding changes in apparent duration. This provides a useful direction for future work. If both retrieval and subjective duration were tested in an experiment that varies the study-test retention interval, for example, would we see a pattern of time-judgments that mimics the forgetting curve? And does providing more retrieval cues at test expand apparent duration as well as improving recall? There are also practical questions: does an eyewitness' report of how long they saw a suspect's face depend on how familiar they are with him or her, for example?

\section{The relevance of subjective time to memory researchers}

Memory researchers have made considerable efforts towards understanding the links between prior exposure and subjective duration, primarily via studies of the fluency heuristic (e.g., Whittlesea, 1993). The results reviewed here show that will be worth pursuing these interrelationships further - and in different directions -- in future.

As one example: the evidence that recent and distant-past exposure have opposite effects on subjective time means that perceived duration may constitute a useful dependent variable when considering whether retention over short and long time intervals rest on distinct structures and 
processes (e.g., Davelaar, Goshen-Gottstein, Ashkenazi, Haarmann, \& Usher, 2005) or are best described with unitary frameworks (e.g., G.D.A. Brown, Neath, \& Chater, 2007; Spurgeon, Ward, \& Matthews, 2014). As noted, systematically charting the time course of the transition from decreasing to increasing subjective duration - ideally using the kinds of verbal material and recall test procedures common to studies of memory -- would provide a useful first step.

Studies of time perception could also inform long-standing debates about the contributions to forgetting of trace decay, interference from intervening or preceding items, and the temporal distinctiveness of the stimuli (G.D.A. Brown \& Lewandowsky, 2010). For example, the temporaldistinctiveness SIMPLE model posits that the discriminability of two items depends on the ratio of their respective retention intervals (G.D.A. Brown et al., 2007). This scalar property is supported by studies of timing, but where Brown and colleagues simply assume a log-transformation of retention interval, time perception researchers offers detailed neural and information-processing mechanisms by which the scalar property arises (e.g., Allman et al., 2014; Hass \& Durstewitz, 2014; Oprisan \& Buhusi, 2014). Moreover, they have identified conditions under which it is violated (e.g., Allman \& Meck, 2012; Grondin, 2014; Wearden \& Lejeune, 2008) - conditions that might therefore produce corresponding changes in retrieval performance, necessitating some adjustment to the memory model.

The processing principle also provides a new perspective on forgetting rates and interference effects. Specifically, the forgetting curve might best be understood in terms of subjective time, with the effects of intervening items on retrieval of a target being mediated by changes in the apparent duration of the retention interval - changes which can be predicted from the processing principle described in the present review. A straightforward way to explore this possibility would be to elicit subjective duration measures for the individual stimuli that fill a retention interval (or judgments of the interval as a whole).

A similar idea applies to the effects of exposure time at the encoding stage. Longer exposure durations boost accuracy in recognition memory tasks (e.g., Tversky \& Sherman, 1975); but perhaps it is apparent, not physical, encoding duration which best predicts performance? According to the processing principle, subjective time is positively related to perceptual clarity and informationextraction and should therefore index the strength and detail of the stimulus representation that underlies subsequent recognition performance. Correspondingly, the effects of basic stimulus properties and encoding conditions that affect recognition (e.g, luminance; Loftus, 1985) will be partly mediated by the apparent duration of the items. Again, this idea could readily be tested by correlating measures of the subjective duration of each item at encoding with subsequent memory performance for that item. We predict that item-level subjective duration would add predictive 
accuracy over and above the effects of above physical exposure duration and macroscopic manipulations.

\section{Section Summary}

We have grouped the effects in this section under the heading 'memory', but they reflect a number of processes including sensory adaptation and attention. More generally, our division of this review into separate sections dealing with 'perception', 'attention', and 'memory' is a convenient organizing device; it is clear by this point that these processes are deeply inter-linked, with complex, inter-connected effects on time perception.

Correspondingly, prior experience with a stimulus has multiple, conflicting effects on subjective duration. Even brief exposure can produce adaptation, reducing the perceptual strength of the adapted stimulus and compressing the apparent duration of repeated features. This is often spatially-specific and short-lived, and probably reflects a basic change in neural sensitivity early in the processing pathway that reduces the effective magnitude of the input - an internal counterpart to the effects of stimulus intensity discussed in Section 2. Similarly, repeated stimuli may seem briefer than novel ones because of early attentional selection of new or unexpected objects: as we saw in Section 2, difference-from-background (salience) provides a useful description of the stimulus properties that expand duration, and repeated items are less distinctive than novel ones. Set against this, stimuli which match an existing mnemonic representation are processed more efficiently. Moreover, repetition can generate the expectation that the item will occur again, a form of cuing effect that, like those surveyed in Section 3, allows the observer to direct resources to the appropriate modality, spatiotemporal location, or region of feature space - boosting perceptual strength and expanding subjective time. These effects are all modulated by expectations and task demands; anticipating stimulus repetition and/or maintaining an active representation in working memory will influence the balance between compressive and expansive effects of past encoding.

This complexity makes it difficult to predict the effects of a given memory/prior-exposure manipulation, but the available data nonetheless suggest that apparent duration tracks perceptual clarity and the ease of information-extraction. That is, prior exposure effects generally accord with the processing principle.

\section{Section 5: Implications and Extensions}

This review has highlighted how the experience of time is intimately connected with other perceptual and cognitive processes, and has offered the processing principle as a useful generalization for conceptualizing these effects and for generating future research questions. In this 
final section we discuss some implications and extensions of the results surveyed above, focusing on (1) extensions to other empirical phenomena, (2) theoretical models of timing, and (3) broader issues regarding the function of subjective time.

\section{Empirical Extensions: Other Determinants of Subjective Time}

The experience of time depends on factors not considered in the body of this review. Of particular interest is the finding that repetitive stimulation - usually a series of auditory 'clicks' presented at the rate of 5 per second for a few seconds - can evoke a generalized change in the apparent duration of subsequent stimuli. Building on work by Treisman, Faulkner, Naish, and Brogan (1990), Penton-Voak, Edwards, Wearden, \& Percival (1996) found that the estimated duration of a light lasting 1183 ms was 760 ms when the stimulus was preceded by 5 -seconds of silence, but 910 ms when it was preceded by 5-seconds of a 5-Hz click-train. Auditory click-trains expand the duration both of auditory and of visual stimuli in a variety of judgment tasks and across species, irrespective of whether the target interval is filled or unfilled, and seems to depend on the frequency of the repetitive stimulation (Cheng, Dyke, McConnell, \& Meck, 2011; Penton-Voak et al., 1996; Wearden et al., 1998, 2007; interestingly, Repp, Mendlowitz, \& Hove, 2013, did not find the clicktrain effect in expert musicians).

Recent work suggests that this expansion of subjective time might be accompanied by more rapid information-processing. Jones, Allely, and Wearden (2011) presented a variety of cognitive tasks, where the start of each trial consisted of a 5-s interval demarcated by two beeps. When this interval was filled with a $5 \mathrm{~Hz}$ click train, subsequent task performance was better than when it was silent or filled with white noise: As well as being faster to detect which of four boxes contained a target and solving mathematics problems more rapidly, participants did better on tasks where performance was not indexed by the speed of a motor response. Specifically, they encoded/retrieved more letters in Sperling's (1960) classic iconic memory task, and pictures that were preceded by clicks were subsequently better recognized in a long-term memory test.

The mechanism for these effects is unclear. Wearden and colleagues posit acceleration of an internal clock (e.g., Penton-Voak et al., 1996), presumably with the additional assumption that the rate of this clock sets the tempo for other mental operations. A potential problem is that more rapid click trains seem to have less effect than slower ones (e.g., Jones et al., 2011). A related possibility is that external stimulation entrains cortical oscillations that might underlie both the experience of time (e.g., McAuley \& Jones, 2003) and other cognitive processes (see Burle, Macar, Bonnet, 2003; Henry \& Herrmann, 2014, for reviews). 
From our perspective, repetitive stimulation provides another instance of the processing principle: improved information extraction corresponds to increased apparent duration. Researchers have not yet combined the time-judgment and cognitive-processing effects of click trains in a single study, but we predict a tight correspondence between the two effects, both across experimental conditions and trial-by-trial during the task. An interesting adjunct to the click-train effect is the recent finding that repetitive stimulation after a stimulus reduces its apparent duration (Ono \& Kitazawa, 2010b, 2011). The reasons are unclear, but the processing principle suggests that the click trains make it harder to extract information from the preceding stimulus, perhaps by displacing relevant representations from working memory. Again, this leads to the testable prediction that non-temporal perceptual judgments about the target stimulus will be impaired in the click-train condition.

More broadly, the processing principle might extend to other pre-stimulus variables that are known to affect apparent duration, such as the change in emotional state that comes from viewing a threatening image (Shi, Jia, \& Müller, 2012) or anticipating an aversive event (Droit-Volet, Mermillod, Cocenas-Silva, \& Gil, 2010). It will be important to test whether the target stimuli are also more/less efficiently processed in these situations, in a way that maps on to the changes in apparent duration. Moreover, some pre-stimulus manipulations reduce the accessibility of subsequent stimuli: emotionally-negative images, for example, can impair the processing of later stimuli in rapid serial visual presentation paradigms (Most, Chun, Widders, \& Zald, 2005); the processing principle implies that the subjective durations of these targets will likewise be compressed. Finally, it will be productive to relate the changes in time judgment that are brought about by physiological and pharmacological manipulations (e.g., Lake \& Meck, 2013) to changes in other perceptual, attentional, and memory processes.

\section{Models of Timing}

How should the links between subjective time and other mental processes be accommodated within theories of human interval timing? We described existing theoretical accounts of specific data above; we now briefly discuss how three general accounts of timing might in future be extended to accommodate the full set of findings.

Pacemaker-accumulator models. Most internal clock models posit a dedicated pacemaker, but the general framework of pulse-accumulation can be separated from this assumption. Indeed, some mathematical models focus on the counting process without explicitly stating the source of the neural pulses (e.g., Creelman, 1962; Rammsayer \& Ulrich, 2001). 
One possibility is that the pulses come from the units representing the stimulus. That is, subjective time might correspond to the accumulated activity of the neurons whose firing forms the 'top level' stimulus-representation. To the extent that stimulus and cognitive variables modulate this representation, they will affect the perception of time - as per the processing principle. Moreover, the assumption that increases in subjective duration would correspond to more neural firing for the relevant stimulus representation is similar to the coding efficiency account of Eagleman and Pariyadath (2009).

A problem with this approach is that it requires the organism to monitor the accumulated activity of all on-going stimulus representations. The physiological implausibility of unlimited accumulation is a problem even for a dedicated pacemaker (Simen, Balci, deSouza, Cohen, and Holmes, 2011b; Simen et al., 2013); it is even more difficult to envisage every stimulus representation having its own accumulator (or that there is a central store for the accumulation of activity across all current representations). Furthermore, as we saw when discussing the coding efficiency hypothesis, there is unlikely to be a simple relationship between processing efficiency, neural firing rates, and subjective duration.

Recent work has sought to connect pacemaker-accumulator models of timing to a broader information-processing framework (van Rijn, Gu, \& Meck, 2014). Specifically, Taatgen, van Rijn, and Anderson (2007) embedded a pacemaker-accumulator timing system the ACT-R architecture (Anderson et al., 2004). ACT-R is an elaborate model that comprises a central procedural memory module connected to a range of processing modules via limited-capacity buffers; it has been used to model a wide range of cognitive tasks, including memory, attention, language processing, and problem-solving (Anderson et al., 2004). Taatgen et al. introduced a 'temporal module' comprising a pacemaker whose noisy inter-pulse intervals increase over time. They were able to model dual-task interference effects on timing by variously assuming interruption of the timing process itself and competition among timing and non-timing tasks for access to declarative memory; they later used ACT-R's memory processes to model the interference between memories for different time intervals (Taatgen \& van Rijn, 2011) and the timing of overlapping intervals (van Rijn \& Taatgen, 2008), although recent work in this area suggests that the model needs some modification (Bryce \& Bratzke, in press; Bryce, Seifried-Dübon, \& Bratzke, 2015; Matthews, 2013.) It is not clear how the ACT-R framework could incorporate the wealth of effects described in this review, but the fact that it has been applied to psychophysical (e.g., Petrov \& Anderson, 2005), attentional (e.g., Anderson, Matessa, \& Lebiere, 1997), and memory (e.g., Pavlik \& Anderson, 2005) processes like those that affect subjective time suggest that, with appropriate assumptions about interactions between the 
timing module and other components of the system, an integrated account might be developed in future.

Oscillator models. A pacemaker-accumulator system is not the only type of dedicated timing mechanism. An alternative class of theory posits oscillator-based timing. In particular, Miall (1989) argued that the duration of an arbitrary interval could be encoded by having a set of neural oscillators, each with a different period, and detecting which subset of these units was active at the time when the interval ended; different intervals would correspond to the coincident activity of different sub-populations of oscillators.

Matell and Meck $(2000,2004)$ developed this idea into a full neurobiological model of interval timing, the striatal beat frequency (SBF) model. In the SBF model, the oscillators are cortical neurons whose phases are reset by dopaminergic input from the ventral tegmental area (VTA) at the onset of a to-be-timed interval. Coincident activity among the oscillators is detected by medium spiny neurons (MSNs) in the striatum; each MSN cell receive a large number of inputs from the cortex and thalamus, and the weight of its synaptic connections with the various oscillators determines the duration that it encodes. The neurobiological structure of the model is supported by a range of electrophysiological, pharmacological, and lesion studies (e.g., Coull, Cheng, \& Meck, 2011; Oprisan \& Buhusi, 2011), as well as psychophysical, brain-imaging, and patient work (e.g., Allman \& Meck, 2012; Hashimoto \& Yotsumoto, 2015; Merchant, Harrington, \& Meck, 2013).

Recent work has drawn connections between the SBF model and the neural mechanisms of working memory (Lustig, Matell, \& Meck, 2005). In particular, oscillating cortical activity has been argued to play a key role in maintaining active working memory representations (e.g., Fuentemilla, Penny, Cashdollar, Bunzeck, \& Düzel, 2010; Lee, Simpson, Logothetis, \& Rainer, 2005; Lisman \& Idiart, 1995; see Roux \& Uhlhaas, 2014, for a recent review), raising the possibility that information about stimulus identity may be encoded by which subset of cortical neurons is firing, while information about stimulus duration is extracted from the pattern of coincident activity. Gu, van Rijn, and Meck (2015) have recently produced a formal model and simulations that illustrate this possibility (Figure 5).

We saw above that apparent duration is expanded for items that are held in working memory, as part of the broader principle that conditions which facilitate perceptual processing lengthen the subjective duration of the stimulus. By modelling timing and working memory in a single framework, the SBF model provides a potential mechanism for this effect. Furthermore, the modulation of cortical oscillations by attention (Jensen, Kaiser, \& Lachaux, 2007) and their links both to the prediction of forthcoming stimuli (Arnal \& Giroud, 2012) and to the formation of coherent object representations (Tallon-Baudry \& Bertrand, 1999) mean that it might be possible to 
incorporate many of the variables that affect subjective time within a single neurobiological model. As yet, this suggestion is purely speculative, but the framework described by Gu et al. (2015) provides a useful starting point.

Sequential sampling models. A different integrative approach would link subjective duration to sequential sampling models, which provide the dominant framework for perceptual decisionmaking. These models predict both choices and response times by assuming that information is successively sampled from the percept to drive the decision process (see Ratcliff \& Smith, 2004, for a review). The most famous example is the drift diffusion model (Ratcliff, 1978; Ratcliff \& McKoon, 2008), wherein sampling drives a time-continuous random walk that terminates when the accumulated information crosses one of two boundaries, each corresponding to a different perceptual decision. The drift rate determines the net rate of information accumulation in favour of one response and depends upon the features of the stimulus (e.g., the signal-to-noise ratio), with a high drift rate entailing fast and accurate decisions and a low rate entailing noisy evidence accumulation and slower, less accurate judgments. The distance between the response boundaries, and the relative location of the starting point between these boundaries, correspond to the decisionmaker's caution and bias, respectively (Figure 6, top).

Drift diffusion models have been applied to a large array of tasks (see Donkin, Brown, Heathcote, \& Wagenmakers, 2011), but historically they have not been linked to temporal judgments. However, Simen, Balci, deSouza, Cohen, and Holmes (2011a) and Rivest and Benjio (2011) have recently and independently developed time-adaptive drift diffusion models (TDDMs) of interval timing (Figure 6, bottom). These models can be conceptualized by imagining a person walking towards a barrier, where each step is perturbed by Gaussian noise. The distance from the start provides the measure of elapsed time; if the steps are small (a low mean drift-rate), the distance will be lower and subjective time will elapse more slowly.

The TDDM models assume that the drift rate adapts so that the barrier is reached at a target time (e.g., so that a lever is pressed at the time when this action will be rewarded), and incorporate the scalar property by assuming that the Gaussian noise increases in proportion to the drift rate (Simen et al., 2011, 2013, provide a neural mechanism for this assumption). The models correctly predict that behavioural response times will follow an inverse Gaussian distribution, with a level of skew that is approximately three times the coefficient of variation (Simen et al., 2013), and can also capture one-trial learning (Simen et al., 2011) and learning of cyclically-varying time intervals (Luzardo, Ludvig, \& Rivest, 2013). These diffusion models therefore accommodate a variety of key findings from timing research within the same basic framework as other forms of perceptual decision-making (albeit with important differences between the two classes of model). 
In the TDDMs, the diffusion process exists purely to time the interval, but might it be possible to develop an integrated account, where the diffusion process that underlies perceptual decisions also provides the basis for the subjective duration of the stimulus? In perceptual decisionmaking, higher drift rates correspond to more efficient extraction of perceptual information, and modelling has shown that the drift rate parameter rises when conditions are favourable for processing (e.g., with high-contrast stimuli or familiar items e.g., Ratcliff, Gomez, \& McKoon, 2004; Ratcliff \& Rouder, 1998); in TDDMs, higher drift rates mean that the process will be further from the start after a given physical interval, corresponding to longer apparent duration. Thus, diffusion models may provide an over-arching mathematical framework for the processing principle described in this review - a framework that quantifiably links responses and reaction times in decision tasks to the precision and accuracy of subjective time.

\section{The Teleology of Subjective Time}

The labiality of duration judgments is often treated, at least implicitly, as a flaw or shortcoming, with non-temporal variables producing 'bias', 'distortions', or 'illusions' (e.g., Eagleman, 2008). While accurate timing is generally desirable, it is worth asking: is the instability of subjective duration an entirely negative and unavoidable consequence of flawed timekeeping, or might it serve - or reflect - some more adaptive process?

This question is particularly apposite for theories that posit a dedicated internal clock. We have seen that researchers frequently attribute the effects of non-temporal variables on subjective time to changes in the rate of a pacemaker, for example. But why should this be? What is the point of a dedicated timing device if all manner of external factors change its accuracy? As we have seen, one possibility is that the clock entails monitoring ongoing internal and external activity and, as such, is inseparable from non-temporal information. Irrespective of this idea, recent work has illustrated the optimality of human and animal timing (Balci, Freestone, \& Gallistel, 2009; Balci et al., 2011; Shi, Church, \& Meck, 2014), and we can ask: Is there some functional value to the flexibility of subjective time?

We briefly consider two possibilities, both of which are grounded in the principle that subjective duration is expanded by conditions that render a stimulus representation more accessible and vivid.

First, apparent duration might index the significance or importance of a stimulus, in the same way that the tingle of fear or the glow of pleasure provides a subjective marker of an item's behavioural relevance. Indeed, this suggestion generalizes the proposal by Droit-Volet and Gil (2009) that the effects of emotion on perceived duration reflect "the excellent ability of the internal clock 
to adapt to events in the environment" (p. 1950). The conditions that expand apparent duration are those that facilitate information extraction, and these are also the conditions that typically signal behavioural significance - for example, proximity, magnitude, uniqueness, and being the focus of attention. This point is especially clear for factors such as looming motion and threatening faces, where behavioural relevance, efficient information processing, and expanded duration go hand in hand. Thus, the feeling that a stimulus lasted for a long time might be a useful subjective index of its importance, one which can combine with other perceptual, affective, and motivational cues to determine the optimum course of action.

A second possibility emphasizes the probability of a given stimulus having a particular physical duration, rather than its utility. The cognitive system is increasingly treated as a Bayesian optimizer that combines noisy sensory input with prior information about the likely state of the world (see, for example, Kersten, Mamassian, \& Yuille, 2004, and Tenenbaum, Kemp, Griffiths, \& Goodman, 2011). In the context of time perception, this approach is exemplified by Jazayeri and Shadlen (2010), who found that temporal reproductions increase linearly with target time, but that they gravitate towards the average of the durations in the experimental session. Jazayeri and Shadlen provided a new perspective on this long-established central tendency by showing that it can be described by a Bayesian model in which the observer integrates a noisy representation of the stimulus with prior information about the stimulus distribution to produce a posterior distribution for the to-be-judged duration, which provides the basis for judgment (Figure 7, top panel). Importantly, the central tendency effect is greatest for the longest duration sets -- the conditions under which timing is most variable, and when it therefore makes most sense to use prior information. Moreover, modelling shows that, when time measurements are less precise because of sensory modality or expertise, the observer uses a narrower prior probability distribution, giving more weight to the mean of the stimulus set when forming a judgment (Cicchini, Arrighi, Cecchetti, Giusti, \& Burr, 2012; see Figure 7, bottom panel).

One possibility, then, is that the shifts in apparent duration caused by non-temporal perception, attention, and memory reflect the combination of noisy time representations with prior information about the probable state of the world. Subjective time would be a 'best guess' about the likely true duration, and factors that expand the apparent duration of a given interval do so because they usually occur when physical duration really is longer. This would be similar to the role of fluency in memory judgments, where "feelings of familiarity are the product of an unconscious interpretive process that attributes fluent processing to a plausible source" (Whittlesea, 1993, p. 1248). Because longer exposure typically improves perceptual representations, the positive association between 
subjective time and information-extraction could reflect a rational perceptual inference in the face of imperfect temporal measurement.

This Bayesian approach could in principle be tested by careful scrutiny of environmental structure. The emergence of wearable technologies that record the sights and sounds people experience each day (e.g., mobile eye-trackers) means that it should be possible to analyse a large body of ecological data to see whether, for example, there is a positive correlation between exposure time and the size or intensity of briefly-viewed objects. Given that larger objects are often slower-moving (and would therefore take longer to become occluded), there might be, but it is impossible to know from first principles. An interesting consideration is that humans partly control the physical duration of the stimuli to which they are exposed - for example, by choosing what to look at or hold on to. These actions facilitate the extraction of information from the stimulus - which we have seen is an over-arching determinant of subjective duration - so subjective time biases could reflect the legitimate prior belief that accessible, perceptually-vivid stimuli are encountered for longer periods of time.

The idea that subjective duration distortions result from best guesses about the state of the world could also be tested by creating micro-environments in the experimental session, where different non-temporal features probabilistically co-occur with longer or shorter intervals. For example, over time, we would expect duration estimates to be shortened for stimuli comprising features that typically predict short exposure, and it should be possible to combine stimulus features with different priors to test whether the integration of prior information is optimal. Moreover, the effects of non-temporal cues should depend both on their reliability (that is, on the variability in the prior) and on the variability of the time interval (greater endogenous or exogenous uncertainty should increase reliance on the non-temporal information; Cicchini et al., 2012) - and both effects could be formally modelled to test the Bayesian framework.

\section{The value of the Processing Principle}

We have suggested that perceptual strength provides the basis for subjective duration: more vivid representations are judged to last longer. This processing principle is a wide-ranging and general precept: it unites the effects on subjective time of stimulus intensity, salience, and magnitude; endogenous and exogenous spatial, temporal, and feature-based attention; expectation, immediate repetition, and long-term memory.

The processing principle has not previously been advanced as a unifying account of subjective time. Rather, the effects of perceptual, attentional, and mnemonic factors on apparent duration have typically been considered in isolation, with specific theories advanced to account for 
specific effects - for example, the notion of a "common magnitude system" that does not speak to the effects of non-magnitude sensory properties, attention, or memory, or an "attentional allocation" model that does not capture the effect of perceptual properties or prior representations. Alternatively, the effects of non-temporal variables have been explained within general-purpose internal-clock frameworks which do not offer any basis for predicting which variables will affect time or how - for example, by asserting that a manipulation "speeds up the internal pacemaker". One strength of the processing principle is that it is both wide-ranging -- encompassing the effects of perceptual properties, attention, and memory -- and specific, in that we can predict the directional influence of a given manipulation by independently investigating how it affects perceptual clarity. Variables that improve detection, identification, and discrimination, and/or which increase the reported vividity of the stimulus representation, are predicted to expand apparent duration. Those which impair information extraction or reduce vividity will have the opposite effect.

This predictive power means that the processing principle provides a useful framework for future work: we can seek new factors that will expand/contract subjective time based on their known effects on perceptual strength. Indeed, the principle generates more specific predictions and questions in the fields of perception, attention, and memory, as described above. More generally, it sets an ambitious agenda for the development of formal models which unify subjective time with the information extraction that underlies performance on other tasks. We have outlined some possibilities above, but imaginative researchers from diverse fields will doubtless be able to develop other ideas.

\section{Conclusion}

Time is a special dimension, but its mental representation is fundamentally linked to other perceptual and cognitive processes. By surveying how subjective time is affected by other stimulus properties, the allocation of processing resources, and the existence of prior stimulus representations, we hope to encourage cross-disciplinary empirical research and integrated theorizing. We have suggested the processing principle as one potentially useful framework for this work, but in any case the empirical links between time, perception, attention, and memory hold the tantalizing prospect of a unified account of temporal and non-temporal cognition. 


\section{References}

Aaen-Stockdale, C., Hotchkiss, J., Heron, J., \& Whitaker, D. (2011). Perceived time is spatial frequency dependent. Vision Research, 51, 1232-1238. doi: 10.1016/j.visres.2011.03.019

Aagten-Murphy, D., Iversen, J. R., Williams, C. L., \& Meck, W. H. (2014). Novel inversions in auditory sequences provide evidence for spontaneous subtraction of time and number. Timing \& Time Perception, 2, 188-209. doi: 10.1163/22134468-00002028

Abrams, R. A., \& Christ, S. E. (2005). The onset of receding motion captures attention: Comment on Franconeri and Simons (2003). Perception \& Psychophysics, 67(2), 219-223.

Abrams, R. A., \& Christ, S. E. (2006). Motion onset captures attention: A rejoinder to Franconeri and Simons (2005). Perception \& Psychophysics, 61(1), 114-117.

Agrillo, C., Ranpura, A., \& Butterworth, B. (2010). Time and numerosity estimation are independent: Behavioral evidence for two different systems using a conflict paradigm. Cognitive Neuroscience, 1(2), 96-101. doi: 10.1080/17588921003632537

Allan, L. G. (1979). The perception of time. Perception \& Psychophysics, 26(5), 340-354.

Allan, L. G., \& Gibbon, J. (1991). Human bisection at the geometric mean. Learning and Motivation, 22, 39-58. doi:10.1016/0023-9690(91)90016-2

Allman, M. J., \& Meck, W. H. (2012). Pathophysiological distortions in time perception and timed performance. Brain, 135, 656-677. doi: 10.1093/brain/awr210

Allman, M. J., Pelphrey, K. A., \& Meck, W. H. (2012). Developmental neuroscience of time and number: Implications for autism and other neurodevelopmental disabilities. Frontiers in Integrative Neuroscience, 6:7. doi: 10.3389/fnint.2012.00007

Allman, M. J., Teki, S., Griffiths, T. D., \& Meck, W. H. (2014). Properties of the internal clock: Firstand second-order principles of subjective time. Annual Review of Psychology, 65, 743-771. doi: 10.1146/annurev-psych-010213-115117

Allman, M. J., Yin, B., \& Meck, W. H. (2014). Time in the psychopathological mind. In D. Lloyd \& V. Arstila (Eds.), Subjective time: The philosophy, psychology, and neuroscience of temporality, pp. 637-654, Cambridge, MA: MIT Press.

Almeida, R., \& Ledberg, A. (2010). A biologically plausible model of time-scale invariant interval timing. Journal of Computational Neuroscience, 28, 155-175. doi: 10.1007/s10827-009-0197-8

Anderson, B. A., Laurent, P. A., \& Yantis, S. (2011). Value-driven attentional capture. Proceedings of the National Academy of Sciences USA, 108(25), 10367-10371. doi: 10.1073/pnas.1104047108

Anderson, J. R., Bothell, D., Byrne, M., Douglass, D., Lebiere, C., \& Qin, Y. (2004). An integrated theory of mind. Psychological Review, 111, 1036-1060. doi: 10.1037/0033-295X.111.4.1036

Anderson, J. R., Matessa, \& Lebiere, C. (1997). ACT-R: A theory of higher level cognition and its relation to visual attention. Human-Computer Interaction, 12, 439-462. doi: 10.1207/s15327051hci1204_5

Anderson, J. R., \& Schooler, L. J. (1991). Reflections of the environment in memory. Psychological Science, 2(6), 396-408. doi: 10.1111/j.1467-9280.1991.tb00174.x

Angrilli, A. Cherubini, P., Pavese, A., \& Manfredini, S. (1997). The influence of affective factors on time perception. Perception \& Psychophysics, 59(6), 972-982.

Arnal, L. H., \& Giraud, A-L. (2012). Cortical oscillations and sensory predictions. Trends in Cognitive Sciences, 16(7), 390-398. 
Aubry, F., Gauillaume, N., Mogicato, G., Bergeret, L., \& Celsis, P. (2008). Stimulus complexity and prospective timing: Clues for a parallel process model of time perception. Acta Psychologica, 128, 63-74. doi: 10.1016/j.actpsy.2007.09.011

Ayhan, I., Bruno, A., Nishida, S., \& Johnston, A. (2009). The spatial tuning of adaptation-based time compression. Journal of Vision, 9(11). Article 2. 1-12. doi: 10.1167/9.11.2

Bacon, W. F., \& Egeth, H. E. (1994). Overriding stimulus-driven attentional capture. Perception \& Psychophysics, 55(5), 485-496.

Balci, F., Freestone, D., \& Gallistel, C. R. (2009). Risk assessment in man and mouse. Proceedings of the National Academy of Sciences, USA, 106(7), 2459-2463. doi: 10.1073/pnas.0812709106

Balci, F., Freestone, D., Simen, P., deSouza, L., Cohen, J. D., \& Holmes, P. (2011). Optimal temporal risk assessment. Frontiers in Integrative Neuroscience, 5, Article 56, 1-15. doi: 10.3389/fnint.2011.00056

Bar-Haim, Y., Kerem, A., Lamy, D., \& Zakay, D. (2010). When time slows down: The influence of threat on time perception in anxiety. Cognition and Emotion, 24(2), 255-263. doi: $10.1080 / 02699930903387603$

Barnes, R., \& Jones, M. R. (2000). Expectancy, attention, and time. Cognitive Psychology, 41, 254311. doi: $10.1006 / \operatorname{cogp} .2000 .0738$

Bartholomew, A. J., Meck, W. H., \& Cirulli, E. T. (2015). Analysis of genetic and non-genetic factors influencing timing and time perception. PLOSONE, in press.

Batty, M. J., Cave, K. R., \& Pauli, P. (2005). Abstract stimuli associated with threat through conditioning cannot be detected preattentively. Emotion, 5(4), 418-430. doi: 10.1037/15283542.5.4.418

Bauer, A-K. R., Jaeger, M., Thorne, J. D., Bendixedn, A., \& Debener, S. (2015). The auditory dynamic attending theory revisited: A closer look at the pitch comparison task. Brain Research, 1626, 198-210. doi: 10.1016/j.brainres.2015.04.032

Bausenhart, K. M., Dyjas, O., \& Ulrich, R. (2014). Temporal reproductions are influenced by an internal reference: Explaining the Vierordt effect. Acta Psychologica, 147, 60-67. doi: 10.1016/j.actpsy.2013.06.011

Bausenhart, K. M., Rolke, B., Seibold, V. C., \& Ulrich, R. (2010). Temporal preparation influences the dynamics of information processing: Evidence for early onset of information accumulation. Vision Research, 50, 1025-1034. doi: 10.1016/j.visres.2010.03.011.

Bausenhart, K. M., Rolke, B., \& Ulrich, R. (2008). Temporal preparation improves temporal resolution: Evidence from constant foreperiods. Perception \& Psychophysics, 70, 1504-1514. doi: 10.3758/PP.70.8.1504.

Berglund, B., Berglund, U., Ekman, G., \& Frankenhaeuser, M. (1969). The influence of auditory stimulus intensity on apparent duration. Scandinavian Journal of Psychology, 10, 21-26. doi: 10.1111/j.1467-9450.1969.tb00003.x

Bi, C., Liu, P., Yuan, X., \& Huang, X. (2014). Working memory modulates the association between time and number representation. Perception, 43, 417-426. doi: 10.1068/p7634

Binetti, N., Lecce, F., \& Doricchi, F. (2012). Time-dilation and time-contraction in an anisochronous and anisometric visual scenery. Journal of Vision, 12, 8, 1-19. doi: 10.1167/12.7.8.

Birngruber, T., Shröter, H., \& Ulrich, R. (2014). Duration perception of visual and auditory oddball stimuli: Does judgment task modulate the temporal oddball effect? Attention, Perception, \& Psychophysics, 76, 814-828. doi: 10.3758/s13414-013-0602-2 
Bjork, R. A., \& Whitten, W. B. (1974). Recency-sensitive retrieval processes in long-term free recall. Cognitive Psychology, 6, 173-189.

Block, R. A. (2003). Psychological timing without a timer: The roles of attention and memory. In $\mathrm{H}$. Helfrich (Ed.), Time and mind II: Information processing perspectives (pp. 41-59). Göttingen, Germany: Hogrefe \& Huber.

Block, R.A., \& Gruber, R. P. (2014). Time perception, attention, and memory: A selective review. Acta Psychologica, 149, 129-133. doi: 10.1016/j.actpsy.2013.11.003

Block, R. A., \& Grondin, S. (2014). Timining and time perception: A selective review and commentary on recent reviews. Frontiners in Psychology, 5, Article 628. 1-3. doi: 10.3389/fpsyg.2014.00648

Block R. A., Hancock P. A. (2013). Time perception. Oxford Online Bibliographies, 284-295. http://www.oxfordbibliographies.com

Block, R. A., Hancock, P. A., \& Zakay, D. (2000). Sex differences in duration judgments: A metaanalytic review. Memory \& Cognition, 28(8), 1333-1346.

Block, R. A., Hancock, P. A., \& Zakay, D. (2010). How cognitive load affects duration judgments: A meta-analytic review. Acta Psychologica, 134, 330-343.

Block, R. A., \& Zakay, D. (1997). Prospective and retrospective duration judgments: A meta-analytic review. Psychonomic Bulletin \& Review, 4(2), 184-197.

Block, R. A., \& Zakay, D. (2008). Timing and remembering the past, the present, and the future. In Grondin, S. (Ed.) Psychology of Time, (pp. 367-394). Bingley: Emerald.

Block, R. A., Zakay, D., \& Hancock, P. A. (1999). Developmental changes in duration judgments: A meta-analytic review. Developmental Review, 19, 183-211.

Bodner, G. E., \& Dypvik, A. T. (2005). Masked priming of number judgments depends on prime validity and task. Memory \& Cognition, 33(1), 29-47.

Bodner, G. E., \& Masson, M. E. J. (2001). Prime validity affects masked repetition priming: Evidence for an episodic resource account of priming. Journal of Memory and Language, 45(4), 616-647. doi: 10.1006/jmla.2001.2791

Bodner, G. E., \& Masson, M. E. J. (2004). Beyond binary judgments: Prime validity modulates masked repetition priming in the naming task. Memory \& Cognition, 32(1), 1-11.

Boltz, M.G. (1992). The remembering of auditory event durations. Journal of Experimental Psychology: Learning, Memory, and Cognition, 18(5), 938-956.

Boltz, M.G. (1993). The generation of temporal and melodic expectancies during musical listening. Perception \& Psychophysics, 53(6), 585-600.

Brannon, E. M., Libertus, M. E., Meck, W. H., \& Woldorff, M. G. (2008). Electrophysiological measures of time processing in infant and adult brains: Weber's law holds. Journal of Cognitive Neuroscience, 20, 193-203. doi: 10.1162/jocn.2008.20016

Brigner, W. L. (1988). Perceived duration as a function of pitch. Perceptual and Motor Skills, 67, 301302. doi: 10.2466/pms.1988.67.1.301

Brown, G. D. A., \& Lewandowsky, S. (2010). Forgetting in memory models: Arguments against trace decay and consolidation failure. In S. Della Sala (Ed.), Forgetting (pp. 49-75). Psychology Press.

Brown, G. D. A., McCormack, T., Smith, M., \& Stewart, N. (2005). Identification and bisection of temporal durations and tone frequencies: Common models for temporal and nontemporal stimuli. Journal of Experimental Psychology: Human Perception and Performance, 31, 919-938.

Brown, G. D. A., Neath, I., \& Chater, N. (2007). A temporal ratio model of memory. Psychological Review, 114, 539-576. 
Brown, S. W. (1995). Time, change, and motion: The effects of stimulus movement on temporal perception. Perception \& Psychophysics, 57, 105-116.

Brown, S. W. (1997). Attentional resources in timing: Interference effects in concurrent temporal and nontemporal working memory tasks. Perception \& Psychophysics, 59, 1118-1140.

Brown, S. W. (2006). Timing and executive function: Bidirectional interference between concurrent temporal production and randomization tasks. Memory \& Cognition, 34, 1464-1471.

Brown, S. W. (2008). Time and attention: Review of the literature. In Psychology of time. S. Grondin (Ed.), (pp. 111-138). Bingley: Emerald.

Brown, S. W. (2014). Involvement of shared resources in time judgment and sequence reasoning tasks. Acta Psychologica, 147, 92-96.

Brown, S. W., Collier, S. A., \& Night, J. C. (2013). Timing and executive resources: Dual-task interference patterns between temporal production and shifting, updating, and inhibition tasks. Journal of Experimental Psychology: Human Perception and Performance, 39(4), 947-963. doi: 10.1037/a0030484

Bruno, A., Ayhan, I. \& Johnston, A. (2010). Retinotopic adaptation-based duration compression. Journal of Vision, 10(10), Article 30, 1-18. doi: 10.1167/10.10.30

Bruno, A., \& Johnston, A. (2010). Contrast gain shapes visual time. Frontiers in Psychology, 1, Article 170, 1-8. doi: 10.3389/fpsyg.2010.00170

Bruno, A., Ng, E., \& Johnston, A. (2013). Motion-direction specificity for adaptation-induced duration compression depends on temporal frequency. Journal of Vision, 13(12), Article 19, 1-11. doi: 10.1167/13.12.19.

Bryce, D., \& Bratzke, D. (in press). Multiple timing of nested intervals: Further evidence for a weighted sum of segments account. Psychonomic Bulletin and Review, doi: 10.3758/s13423015-0877-5

Bryce, D., Seifried-Dübon, T., \& Bratzke, D. (2015). How are overlapping time intervals perceived? Evidence for a weighted sum of segments model. Acta Psychologica, 156, 83-95. doi: 10.1016/j.actpsy.2014.12.007.

Bueti, D., \& Buonomano, D. (2014). Temporal perceptual learning. Timing \& Time Perception, 2, 261289. doi: $10.1163 / 22134468-00002023$

Bueti, D., \& Walsh, V. (2009). The parietal cortex and the represetation of time, space, number and other magnitudes. Philosophical Transactions of The Royal Society B, 364, 1831-1840. doi: 10.1098/rstb.2009.0028

Buhusi, C. V., Aziz, D., Winslow, D., Carter, R. E., Swearingen, J. E., \& Buhusi, M. C. (2009). Interval timing accuracy and scalar timing in c57BL/6 mice. Behavioral Neuroscience, 123, 1102-1113.

Buhusi, C. V., \& Cordes, S. (2011). Time and number: The privileged status of small values in the brain. Frontiers in Integrative Neuroscience, 5:67. doi: 10.3389/fnint.2011.00067

Buhusi, C. V., \& Meck, W. H. (2005). What makes us tick? Functional and neural mechanisms of interval timing. Nature Reviews Neuroscience, 6, 755-765. doi:10.1038/nrn1764

Buhusi, C. V., \& Meck, W. H. (2009). Relative time sharing: New findings and an extension of the resource allocation model of temporal processing. Philosophical Transactions of the Royal Society $B, 364,1875-1885$. doi: $10.1098 /$ rstb.2009.0022

Buhusi, C. V., \& Oprisan, S. A. (2013). Time-scale invariance as an emergent property in a perceptron with realistic, noisy neurons. Behavioural Processes, 95, 60-70. doi:

10.1016/j.beproc.2013.02.015 
Buonomano, D. V. (2007). The biology of time across different scales. Nature Chemical Biology, 3, 594-597. doi:10.1038/nchembio1007-594

Buonomano, D. V. (2014). Neural dynamics based timing in the subsecond to seconds range. Advances in Experimental Medicine and Biology, 829, 101-117. doi: 10.1007/978-1-4939-1782$-6$

Buonomano, D.V., Bramen, J., \& Khodadadifar, M. (2009). Influence of the interstimulus interval on temporal processing and learning: Testing the state-dependent network model. Philosophical Transactions of the Royal Society B, 364, 1865-1873. doi: 10.1098/rstb.2009.0019

Buonomano, D.V. \& Laje, R. (2010). Population clocks: Motor timing with neural dynamics. Trends in Cognitive Sciences, 14(12), 520-527. doi: 10.1016/j.tics.2010.09.002

Burle, B., Macar, F., \& Bonnet, M. (2003). Behavioral and electrophysiological oscillations in information processing: A tentative synthesis. In H. Helfrich (Ed.), Time and mind: Il. Information processing perspectives (pp. 209-232). Hildesheim, Germany: Hogrefe and Huber.

Burr, D. C., Cicchini, G. M., Arrighi, R., \& Morrone, M. C. (2011). Spatiotopic selectivity of adaptationbased compression of event duration. Journal of Vision, 11(2), Article 21, 1-9. doi: 10.1167/11.2.21.

Burr, D., Tozzi, A., \& Morrone, M. C. (2007). Neural mechanisms for timing visual events are spatially selective in real-world coordinates. Nature Neuroscience, 10(4), 423-425.

Burton, H., Abend, N. S., MacLeod, A-M. K., Sinclair, R. J., Snyder, A. Z., \& Raichle, M. E. (1999). Tactile attention tasks enhance activation in somatosensory regions of parietal cortex: $A$ positron emission tomography study. Cerebral Cortex, 9, 662-674.

Cai, Z. G., \& Wang, R. (2014). Numerical magnitude affects temporal memories but not time encoding. PLOS ONE, 9(1), Article e83159, 1-9.

Callender, C. (2010). Is time an illusion? Scientific American - Mind, 23, 14-21. doi: 10.1038/scientificamericantime1114-14

Calvo, M., \& Esteves, F. (2005). Detection of emotional faces: Low perceptual threshold and wide attentional span. Visual Cognition, 12(1), 13-27. doi: 10.1080/13506280444000094

Capizzi, M., Correa, Á. \& Sanabria, D. (2013). Temporal orienting of attention is interfered by concurrent working memory updating. Neuropsychologia, 51, 326-339. doi: 10.1016/j.neuropsychologia.2012.10.005

Capizzi, M., Sanabria, D., \& Correa, Á. (2012). Dissociating controlled from automatic processing in temporal preparation. Cognition, 123, 293-302. doi: 10.1016/j.cognition.2012.02.005

Cappelletti, M., Freeman, E. D., \& Butterworth, B. L. (2011). Time processing in dyscalculia. Frontiers in Psychology, 2, Article 364, 1-10. doi: 10.3389/fpsyg.2011.00364

Cappelletti, M., Freeman, E. D., \& Cipolotti, L. (2011). Numbers and time doubly dissociate. Neuropsychologia, 49, 3078-30992. doi: 10.1016/j.neuropsychologia.2011.07.014

Carey, S. (2009). Where our number concepts come from. Journal of Philosophy, 106(4), 220-254.

Carrasco, M. (2011). Visual attention: The past 25 years. Vision Research, 51, 1484-1525. doi: 10.1016/j.visres.2011.04.012

Carrozzo, M., Moscatelli, A., \& Lacquaniti, F. (2010). Tempo rubato: Animacy speeds up time in the brain. PLOS ONE, 5(12), e15638, 1-13. doi: 10.1371/journal.pone.0015638

Casini, L., \& Macar, F. (1997). Effects of attention manipulation on judgments of duration and of intensity in the visual modality. Memory \& Cognition, 25, 812-818.

Casini, L., \& Vidal, F. (2011). The SMAs: neural substrate of the temporal accumulator? Frontiers in Integrative Neuroscience, 5, Article 35, 1-3. doi: 10.3389/fnint.2011.00035 
Champagne, J., \& Fortin, C. (2008). Attention sharing during timing: Modulation by processing demands of an unexpected stimulus. Perception \& Psychophysics, 70(4), 630-639. doi: 10.3758/PP.70.4.630

Chang, A. Y-C., Tzeng, O. J. L., Hung, D. L., \& Wu, D. H. (2011). Psychological Science, 22(12), 15671573. doi: $10.1177 / 0956797611418837$

Chater, N., \& Brown, G. D. A. (1999). Scale-invariance as a unifying psychological principle. Cognition, 69, B17-B24.

Chen, Z., \& O'Neill, P. (2001). Processing demand modulates the effects of spatial attention on the judged duration of a brief stimulus. Perception \& Psychophysics, 63, 1229-1238.

Cheng, R. K., Dyke, A. G., McConnell, M. W., \& Meck, W. H. (2011). Categorical scaling of duration as a function of temporal context in aged rats. Brain Research, 1381, 175-186. doi: 10.1016/j.brainres.2011.01.044

Chica, A. B., \& Christie, J. (2009). Spatial attention does improve temporal discrimination. Attention, Perception, \& Psychophysics, 71, 273-280.

Chun, M. M., Golomb, J. D., \& Turk-Browne, N. B. (2011). A taxonomy of external and internal attention. Annual Review of Psychology, 62, 73-101. doi: 10.1146/annurev.psych.093008.100427.

Chun, M., \& Jiang, Y. (1998). Contextual cueing: Implicit learning and memory of visual context guides spatial attention. Cognitive Psychology, 36(1), 28-71. doi: 10.1006/cogp.1998.0681

Church, R. M., \& Gibbon, J. (1982). Temporal generalization. Journal of Experimental Psychology: Animal Behavior Processes, 8(2), 165-186. doi: 10.1037/0097-7403.8.2.165

Cicchini, G. M., Arrighi, R., Cecchetti, L., Giusti, M., \& Burr, D. C. (2012). Optimal encoding of interval timing in expert percussionists. Journal of Neuroscience, 32, 1056-1060. doi: 10.1523/JNEUROSCI.3411-11.2012

Clark, A. (2013). Whatever next? Predictive brains, situation agents, and the future of cognitive science. Behavioral and Brain Sciences, 36, 181-253. doi: 10.1017/S0140525X12000477

Clifford, C. W. G. (2002). Perceptual adaptation: Motion parallels orientation. Trends in Cognitive Sciences, 6, 136-143. doi: 10.1016/S1364-6613(00)01856-8

Cocenas-Silva, R., Bueno, J. L., Doyère, V., \& Droit-Volet, S. (2013). Emotion and long-term memory for duration: Resistance against interference. Behavioural Processes, 97, 6-10. doi: 10.1016/j.beproc.2013.03.010

Cocenas-Silva, R., Bueno, J. L., Doyère, V., \& Droit-Volet, S. (2014). Memory consolidation for duration. Quarterly Journal of Experimental Psychology, 67(7), 1401-1414. doi. 10.1080/17470218.2013.863375

Cohen, J., Hansel, C. E. M., \& Sylvester, J. D. (1954). Interdependence of temporal and auditory judgments. Nature, 174, 642-644. doi:10.1038/174642a0

Corbetta, M., \& Shulman, G. L. (2002). Control of goal-directed and stimulus-driven attention in the brain. Nature Reviews Neuroscience, 3, 201-215. doi:10.1038/nrn755

Cordes, S., Williams, C. L., \& Meck, W. H. (2007). Common representations of abstract quantities. Current Directions in Psychological Science, 16, 156-161. doi: 10.1111/j.1467-8721.2007.00495.x

Correa, Á., Lupiáñez, J., Milliken, B., \& Tudela, P. (2004). Endogenous temporal orienting of attention in detection and discrimination tasks. Psychonomic Bulletin \& Review, 66(2), 264-278.

Correa, Á., Sanabria, D., Spence, C., Tudela, P., \& Lupiáñez, J. (2006). Selective temporal attention enhances the temporal resolution of visual perception: Evidence from a temporal order judgment task. Brain Research, 1070, 202-205. doi: 10.1016/j.brainres.2005.11.094 
Coull, J. T., Cheng, R. K., \& Meck, W. H. (2011). Neuroanatomical and neurochemical substrates of timing. Neuropsychopharmacology 36, 3-25. doi: 10.1038/npp.2010.113

Coull, J. T., Frith, C. D., Büchel, C., \& Nobre, A. C. (2000). Orienting attention in time: Behavioural and neuroanatomical distinction between exogenous and endogenous shifts. Neuropsychologia, 38, 808-819. doi: 10.1016/S0028-3932(99)00132-2

Coull, J. T., \& Nobre, A. C. (1998). Where and when to pay attention: The neural systems for directing attention to spatial locations and to time intervals as revealed by both PET and fMRI. Journal of Neuroscience, 18, 7426-7435.

Coull, J. T., \& Nobre, A. C. (2008). Dissociating explicit timing from temporal expectation with fMRI. Current Opinion in Neurobiology, 18, 137-144. doi: 10.1016/j.conb.2008.07.011

Coull, J. T., Vidal, F., Nazarian, B., \& Macar. F. (2004). Functional anatomy of the attentional modulation of time estimation. Science, 303, 1506-1508. doi: 10.1126/science.1091573

Cravo, A. M., Rohenkohl, G., Wyart, V., \& Nobre, A.C. (2013). Temporal expectation enhances contrast sensitivity by phase entrainment of low-frequency oscillations in visual cortex. Journal of Neuroscience, 33(9), 4002-4010. doi: 10.1523/JNEUROSCI.4675-12.2013

Creelman, C. D. (1962). Human discrimination of auditory duration. Journal of the Acoustical Society of America, 34(5), 582-593.

Curran, W., \& Benton, C. P. (2012). The many directions of time. Cognition, 122, 252-257. doi: 10.1016/j.cognition.2011.10.016

Dallal, N. L., Yin, B., Nekovářová, T., Stuchlík, A., \& Meck, W. H. (2015). Vestibular inputs to spatial navigation and interval timing: The role of self-initiated motion in spatial-temporal integration. Timing \& Time Perception, 3-4, 1-36. doi: 10.1163/22134468-03002053

Davelaar, E. J., Goshen-Gottstein, Y., Ashkenazi, A., Haarmann, H. J., \& Usher, M. (2005). The demise of short-term memory revisited: Empirical and computational investigations of recency effects. Psychological Review, 112(1), 3-42. doi: 10.1037/0033-295X.112.1.3

De Baene, W., \& Vogels, R. (2010). Effects of adaptation on the stimulus selectivity of macaque inferior temporal spiking activity and local field potentials. Cerebral Cortex, 20(9), 2145-2165. doi: 10.1093/cercor/bhp277

De Corte, B. J., \& Matell, M. S. (2015). Temporal averaging across multiple response options: Insight into the mechanisms underlying integration. Animal Cognition, doi: 10.1007/s10071-015-0935-4

de Haas, B., Cecere, R., Cullen, H., Driver, J., \& Romei, V. (2013). The duration of a co-occuring sound modulates visual detection performance in humans. PLOS ONE, 8(1), Article e54789, 1-8. doi: 10.1371/journal.pone.0054789

Devane, J. R. (1974). Word characteristics and judged duration for two response sequences. Perceptual and Motor Skills, 38, 525-526.

Donkin, C., Brown, S., Heathcote, A., \& Wagenmakers, E. J. (2011). Diffusion versus linear ballistic accumulation: Different models but the same conclusions about psychological processes? Psychonomic Bulletin \& Review, 18, 61-69. doi: 10.3758/s13423-010-0022-4.

Dormal, V., \& Pesenti, M. (2007). Numerosity-length interference. Experimental Psychology, 54(4), 289-297.

Dormal, V., \& Pesenti, M. (2012). Processing magnitudes within the parietal cortex. In A. Costa \& E. Villalba (Eds.), Horizons in neuroscience research, Vol. 8, (pp. 107-140). New York, NY: Nova Science Publishers. 
Dormal, V., \& Pesenti, M. (2013). Processing numerosity, length and duration in a three-dimensional Stroop-like task: Towards a gradient of processing automaticity? Psychological Research, 77, 16127.

Dormal, V., Seron, X., \& Pesenti, M. (2006). Numerosity-duration interference: A Stroop experiment. Acta Psychologica, 121, 109-124. doi: 10.1016/j.actpsy.2005.06.003

Droit-Volet, S. (2002). Scalar timing in temporal generalization in children with short and long stimulus durations. Quarterly Journal of Experimental Psychology, 55A(4), 1193-1209. doi: $10.1080 / 02724980244000161$

Droit-Volet, S. (2003). Temporal experience and timing in children. In W.H. Meck (Ed.), Functional and neural mechanisms of interval timing. (pp. 183-208). Boca Raton, FL: CRC Press.

Droit-Volet, S. (2008). A further investigation of the filled-duration illusion with a comparison between children and adults. Journal of Experimental Psychology: Animal Behavior Processes, 34(3), 400-414. doi: 10.1037/0097-7403.34.3.400

Droit-Volet, S. (2010a). Speeding up a master clock common to time, number and length? Behavioural Processes, 85, 126-134. doi: 10.1016/j.beproc.2010.06.017

Droit-Volet, S. (2010b). Stop using time reproduction tasks in a comparative perspective without further analyses of the role of the motor response: The example of children. European Journal of Cognitive Psychology, 22(1), 130-148. doi: 10.1080/09541440902738900

Droit-Volet, S. (2012). Children and time. The Psychologist, 25, 586-589.

Droit-Volet, S., Brunot, S., \& Neidenthal, P. (2004). Perception of the duration of emotional events. Cognition and Emotion, 18(6), 849-858. doi: 10.1080/02699930341000194

Droit-Volet, S., \& Gil, S. (2009). The time-emotion paradox. Philosophical Transactions of the Royal Society B, 364, 1943-1953. doi: 10.1098/rstb.2009.0013

Droit-Volet, S., Meck, W. H., \& Penney, T. B. (2007). Sensory modality and time perception in children and adults. Behavioural Processes, 74, 244-250. doi: 10.1016/j.beproc.2006.09.012

Droit-Volet, S., Mermillod, M., Cocenas-Silva, R., \& Gil, S. (2010). The effect of expectancy of a threatening event on time perception in human adults. Emotion, 10(6), 908-914. doi: $10.1037 / \mathrm{a} 0020258$

Droit-Volet, S., \& Zélanti, P. S. (2013). Development of time sensitivity and information processing speed. PLOS ONE, 8(8), e71424, 1-9. doi: 10.1371/journal.pone.0071424

Dutilh, G., Krypotos, A-M., \& Wagenmakers, E-J. (2011). Task-related versus stimulus-specific practice: A diffusion model account. Experimental Psychology, 58(6), 434-442. doi: 10.1027/1618-3169/a000111

Dyjas, O., Bausenhart, K. M., \& Ulrich, R. (2012). Trial-by-trial updating of an internal reference in discrimination tasks: Evidence from effects of stimulus order and trial sequence. Attention, Perception, \& Psychophysics, 74(8), 1819-1841.

Dyjas, O., \& Ulrich, R. (2014). Effects of stimulus order on discrimination processes in comparative and equality judgments: Data and models. Quarterly Journal of Experimental Psychology, 67(6), 1121-1150. doi: 10.1080/17470218.2013.847968

Eagleman, D. M. (2008). Human time perception and its illusions. Current Opinion in Neurobiology, 18, 131-136. doi: 10.1016/j.conb.2008.06.002

Eagleman, D. M., \& Pariyadath, V. (2009). Is subjective duration a signature of coding efficiency? Philosophical Transactions of the Royal Society B, 364, 1841-1851. doi: 10.1098/rstb.2009.0026 
Eimer, M., Kiss, M., Press, C., \& Sauter, D. (2009). The roles of feature-specific task set and bottomup salience in attentional capture: An ERP study. Journal of Experimental Psychology: Human Perception and Performance,35(5), 1316-1328. doi: 10.1037/a0015872

Ekman, G., Frankenhaeuser, M., Berglund, B., \& Waszak, M. (1969). Apparent duration as a function of intensity of vibrotactile stimulation. Perceptual and Motor Skills, 28, 151-156.

Ekman, G., Frankenhaeuser, M., Levander, S., \& Mellis, I. (1966). The influence of intensity and duration of electrical stimulation on subjective variable. Scandinavian Journal of Psychology, 7, 58-64. doi: 10.1111/j.1467-9450.1966.tb01338.x

Enns, J. T., Brehaut, J. C., \& Shore, D. I. (1999). The duration of a brief event in the mind's eye. Journal of General Psychology, 126, 355-372. doi: 10.1080/00221309909595371

Fayolle, S.L., \& Droit-Volet, S. (2014). Time perception and dynamics of facial expressions of emotions. PLoS ONE, 9(5), Article e97944, 1-9. doi: 10.1371/journal.pone.0097944

Fernandez, D., \& Wilkins, A. J. (2008). Uncomfortable images in art and nature. Perception, 37(7), 1098-1113.

Field, D. J., \& Brady, N. (1997). Visual sensitivity, blur and the sources of variability in the amplitude spectra of natural scenes. Vision Research, 37(23), 3367-3383.

Folta-Schoofs, K., Wolf, O. T., Treue, S., \& Schoofs, D. (2014). Perceptual complexity, rather than valence or arousal accounts for distracter-induced overproductions of temporal durations. Acta Psychologica, 147, 51-59. doi: 10.1016/j.actpsy.2013.10.001

Fortin, C. (1999). Short-term memory in time interval production. International Journal of Psychology, 34(5/6), 308-316. doi: 10.1080/002075999399611

Fortin, C., Fairhurst, S., Malapani, C., Morin, C., Towey, J., \& Meck, W. H. (2009). Expectancy in humans in multisecond peak-interval timing with gaps. Attention, Perception \& Psychophysics, 71, 789-802. doi: 10.3758/APP.71.4.789

Fortin, C., \& Masse, N. (2000). Expecting a break in time estimation: Attentional time-sharing without concurrent processing. Journal of Experimental Psychology: Human Perception and Performance, 26(6), 1788-1796. doi: 10.1037/0096-1523.26.6.1788

Fortin, C., Schweickert, R., Gaudreault, R., \& Viau-Quesnel, C. (2010). Timing is affected by demand in memory search but not by task switching. Journal of Experimental Psychology: Human Perception and Performance, 36(3), 580-595. doi: 10.1037/a0017639

Fortin, C., \& Tremblay, S. (2006). Interrupting timing in interval production and discrimination: Similarities and differences. Behavioural Processes, 71, 336-343. doi: 10.1016/j.beproc.2005.10.003

Fox, E., Russo, R., \& Dutton, K. (2002). Attentional bias for threat: Evidence for delayed disengagement from emotional faces. Cognition and Emotion, 16(3), 355-379. doi: 10.1080/02699930143000527

Franconeri, S. L., \& Simons, D. J. (2003). Moving and looming stimuli capture attention. Perception \& Psychophysics, 65(7). 999-1010.

Franconeri, S. L., \& Simons, D. J. (2005). The dynamic events that capture visual attention: A reply to Abrams and Christ (2005). Perception \& Psychophysics, 67(6). 962-966.

Franssen, V., \& Vandierendonck, A. (2002). Time estimation: Does the reference memory mediate the effect of knowledge of results? Acta Psychologica, 109, 239-267. doi: 10.1016/S00016918(01)00059-2

Friston, K. (2005). A theory of cortical responses. Philosophical Transactions of the Royal Society B: Biological Sciences, 360, 815-836. doi: 10.1098/rstb.2005.1622 
Fuentemilla, L., Penny, W., Cashdollar, N., Bunzeck, N., \& Düzel, E. (2010). Theta-coupled periodic reply in working memory. Current Biology, 20, 606-612. doi: 10.1016/j.cub.2010.01.057

Gamache, P-L., Grondin, S., \& Zakay, D. (2011). The impact of attention in the internal clock in prospective timing: Is it direct or indirect? Lecture Notes in Computer Science, 6789, 137-150. doi: 10.1007/978-3-642-21478-3 12

Gaureault, R., Fortin, C., \& Macar, F. (2010). Contrasting effects of interference and of breaks in interval timing. Acta Psychologica, 133, 3-16. doi: 10.1016/j.actpsy.2009.07.013

Gibbon, J. (1977). Scalar expectancy theory and Weber's law in animal timing. Psychological Review, 84, 279-325. doi: 10.1037/0033-295X.84.3.279

Gibbon, J. (1992). Ubiquity of scalar timing with a Poisson clock. Journal of Mathematical Psychology, 35, 283-293. doi: 10.1016/0022-2496(92)90041-5

Gibbon, J., Church, R. M., \& Meck, W. H. (1984). Scalar timing in memory. Annals of the New York Academy of Sciences, 423, 52_77. doi: 10.1111/j.1749-6632.1984.tb23417.x

Gibbon, J., Malapani, C., Dale, C. L., \& Gallistel, C. R. (1997). Toward a neurobiology of temporal cognition: Advances and challenges. Current Opinion in Neurobiology, 7(2), 170-184. doi: 10.1016/S0959-4388(97)80005-0

Gil, S. \& Droit-Volet, S. (2011). "Time flies in the presence of angry faces..." depending on the temporal task used! Acta Psychologica, 136, 354-362. doi: 10.1016/j.actpsy.2010.12.010

Gil, S., \& Droit-Volet, S. (2012). Emotional time distortions: The fundamental role of arousal. Cognition and Emotion, 26(5), 847-862. doi: 10.1080/02699931.2011.625401

Gil, S., Niedenthal, P. M., \& Droit-Volet, S. (2007). Anger and time perception in children. Emotion, 7(1), 219-225. doi: 10.1037/1528-3542.7.1.219

Glasser, D. M., Tsui, J. M. G., Pack, C. C., \& Tadin, D. (2011). Perceptual and neural consequences of rapid motion adaptation. Proceedings of the National Academy of Sciences USA, 108, E10801088. doi: $10.1073 /$ pnas. 1101141108

Goel, A., \& Buonomano, D.V. (2014). Timing as an intrinsic property of neural networks: Evidence from in vivo and in vitro experiments. Philosophical Transactions of the Royal Society B: Biological Sciences, 369, 20120460, doi: 10.1098/rstb.2012.0460

Goldstone, S., \& Goldfarb, J. L. (1964). Auditory and visual time judgment. Journal of General Psychology, 70, 369-387.

Goldstone, S., \& Lhamon, W. T. (1974). Studies of auditory-visual differences in human time judgment: 1 . Sounds are judged longer than lights. Perceptual and Motor Skills, 39, 63-82.

Goldstone, S., Lhamon, W. T., \& Sechzer, J. (1978). Light intensity and judged duration. Bulletin of the Psychonomic Society, 12(1), 83-84.

Gomez, L. M., \& Robertson, L. C. (1979). The filled-duration illusion: The function of temporal and non-temporal set. Perception \& Psychophysics, 25(5), 432-438.

Gooch, C. M., Stern, Y., \& Rakitin, B. C. (2009). Evidence for age-related changes to temporal attention and memory from the choice time production task. Neuropsychology Development Cognition B Aging Neuropsychology Cognition, 16(3), 285-310. doi: 10.1080/13825580802592771

Gorea, A. (2011). Ticks per thought or thoughts per tick? A selective review of time perception with hints on future research. Journal of Physiology - Paris, 105, 153-163. doi: 10.1016/j.jphysparis.2011.09.008

Gorea, A., \& Kim, J. (2015). Time dilates more with apparent speed than with physical speed. Journal of Vision, 15(1), Article 7, 1-11. doi: 10.1167/15.1.7 
Grassi, M., \& Darwin, C.J. (2006). The subjective duration of ramped and damped sounds. Perception \& Psychophysics, 68(8), 1382-1392.

Grassi, M., \& Pavan, A. (2012). The subjective duration of audiovisual looming and receding stimuli. Attention, Perception, \& Psychophysics, 74, 1321-1333. doi: 10.3758/s13414-012-0324-x

Grill-Spector, K., Henson, R., \& Martin, A. (2006). Repetition and the brain: Neural models of stimulus-specific effects. Trends in Cognitive Sciences, 10, 14-23. doi:10.1016/j.tics.2005.11.006

Grommet, E. K., Droit-Volet, S., Gil, S., Hemmes, N. S., Baker, A. H., \& Brown, B. L. (2010). Time estimation of fear cues in human observers. Behavioural Processes, 86, 88-93. doi: 10.1016/j.beproc.2010.10.003

Grondin, S. (2001). From physical time to the first and second moments of psychological time. Psychological Bulletin, 127, 22-44. doi: 10.1037/0033-2909.127.1.22

Grondin, S. (Ed.) (2008). Psychology of Time. Bingley: Emerald.

Grondin, S. (2010). Timing and time perception: A review of recent behavioral and neuroscience findings and theoretical directions. Attention, Perception, \& Psychophysics, 72(3), 561-582.

Grondin, S. (2010). Unequal Weber fractions for the categorization of brief temporal intervals. Attention, Perception, \& Psychophysics, 72, 1422-1430.

Grondin, S. (2012). Violation of the scalar property for time perception between 1 and 2 seconds: Evidence from interval discrimination, reproduction, and categorization. Journal of Experimental Psychology: Human Perception and Performance, 38, 880-890. doi: 10.1037/a0027188

Grondin, S. (2014). About the (non)scalar property for time perception. Advances in Experimental Medicine and Biology, 829, 17-32. doi: 10.1007/978-1-4939-1782-2_2

Grondin, S., Meilleur, G., \& Lachance, R. (1999). When to start explicit counting in a time-intervals discrimination task: A critical point in the timing process of humans. Journal of Experimental Psychology: Human Perception and Performance, 25(4), 993-1004. doi: 0.1037/00961523.25.4.993

Grondin, S., Ouellet, B., \& Roussel, M-E. (2004). Benefits and limits of explicit counting for discrimination temporal intervals. Canadian Journal of Experimental Psychology, 58(1), 1012. doi: $10.1037 /$ h0087436

Grondin, S., \& Rammsayer, T. (2003). Variable foreperiods and temporal discrimination. Quarterly Journal of Experimental Psychology, 56A, 731-765. doi: 10.1080/02724980244000611

Gu, B-M., Jurkowski, A. J., Lake, J. I., Malapani, C., \& Meck, W. H. (2015). Bayesian models of interval timing and distortions in temporal memory as a function of Parkinson's disease and dopaminerelated error processing. In A. Vatakis \& M.J. Allman (Eds.), Time distortions in mind: Temporal processing in clinical populations. (pp. 284-329). Boston, MA: Brill Academic Publishers.

Gu, B. M., \& Meck, W. H. (2011). New perspectives on Vierordt's law: Memory-mixing in ordinal temporal comparison tasks. Multidisciplinary Aspects of Time and Time Perception: Lecture Notes in Computer Science, 6789, 67-78. doi: 10.1007/978-3-642-21478-3_6

Gu, B-M., van Rijn, H., \& Meck, W. H. (2015). Oscillatory multiplexing of neural population codes for interval timing and working memory. Neuroscience and Biobehavioral Reviews, 48, 160-185. doi: 10.1016/j.neubiorev.2014.10.008

Hackley, S. A. (2009). The speeding of voluntary reaction by a warning signal. Psychophysiology, 46, 225-233. doi: 10.1111/j.1469-8986.2008.00716.x

Hancock, P. A., \& Rausch, R. (2010). The effects of sex, age, and interval duration on the perception of time. Acta Psychologica, 133(2), 170-179. doi: 10.1016/j.sctpsy.2009.11.005 
Harrington, D. L., Castillo, G. N., Fong, C. H., \& Reed, J. D. (2011). Neural underpinnings of distortions in the experience of time across the senses. Frontiers in Integrative Neuroscience, 5, Article 32, 1-14 doi: 10.3389/fnint.2011.00032

Hashimoto, Y., \& Yotsumoto, Y. (2015). Effect of temporal frequency spectra of flicker on time perception: Behavioral testing and simulations using a striatal beat-frequency model. Timing \& Time Perception. 3-4, xx-xx. doi: 10.1163/22134468-03002049

Hass, J., \& Durstewitz, D. (2014). Neurocomputational models of time perception. Advances in Experimental Medicine and Biology, 828, 49-71. doi: 10.1007/978-1-4939-1782-2_4

Hasuo, E., Nakajima, Y., \& Ueda, K. (2011). Does filled duration illusion occur for very short tie intervals. Acoustical Science and Technology, 32(2), 82-85. doi: 10.1250/ast.32.82

Haun, D. B. M., Jordan, F. M., Vallortigara, G., \& Clayton, N. S. (2010). Origins of spatial, temporal and numerical cognition: Insights from comparative psychology. Trends in Cognitive Science, 14(12), 552-560. doi: 10.1016/j.tics.2010.09.006.

Hayashi, M. J., Kanai, R., Tanabe, H. C., Yoshida, Y., Carlson, S., Walsh, V., \& Sadato, N. (2013). Interaction of numerosity and time in prefrontal and parietal cortex. Journal of Neuroscience, 33(3), 883-893. doi: 10.1523/JNEUROSCI.6257-11.2013.

Hayashi, M. J., Valli, A., \& Carlson, S. (2013). Neuroscience Letters, 543, 7-11. doi: 10.1016/j.neulet.2013.02.054

Hein, E., Rolke, B., \& Ulrich, R. (2006). Visual attention and temporal discrimination: Differential effects of automatic and voluntary cueing. Visual Cognition, 13, 29-50.

Hellström, Å. (1979). Time errors and differential sensation weighting. Journal of Experimental Psychology: Human Perception and Performance, 5, 460-477.

Hellström, Å. (2003). Comparison is not just subtraction: Effects of time- and space-order on subjective stimulus difference. Perception \& Psychophysics, 65, 1161-1177.

Henry, M.J., \& Herrmann, B. (2014). Low-frequency neural oscillations support dynamic attending in temporal context. Timing \& Time Perception, 2, 62-86. doi: 10.1163/22134468-00002011

Henson, R. N. A., \& Rugg, M. D. (2003). Neural response suppression, haemodynamic repetition effects, and behavioural priming. Neuropsychologia, 41, 263-270. doi:10.1016/S00283932(02)00159-8

Herbst, S. K., Javadi, A. H., van der Meer, E., \& Busch, N. A. (2013). How long depends on how fast Perceived flicker dilates subjective duration. PLOS ONE, 8(10), e76074, 1-11. doi:

10.1371/journal.pone.0076074

Hicks, R. E., Miller, G. W., \& Kinsbourne, M. (1976). Prospective and retrospective judgments of time as a function of amount of information processed. American Journal of Psychology, 89(4), 719730.

Hinton, S. C, \& Meck, W. H. (2004). Frontal-striatal circuitry activated by human peak-interval timing in the supra-seconds range. Cognitive Brain Research, 21, 171-182. doi:10.1016/j.cogbrainres.2004.08.005

Hillyard, S. A., Vogel, E. K., \& Luck, S. J. (1998). Sensory gain control (amplification) as a mechanism of selective attention: Electrophysiological and neuroimaging evidence. Philosophical Transactions of the Royal Society of London B, 353, 1257-1270.

Hogan, H. W. (1975). Time perception and stimulus preference as a function of stimulus complexity. Journal of Personality and Social Psychology, 31(1), 32-35. 
Horr, N. K., \& Di Luca, M. (2015). Taking a long look at isochrony: Perceived duration increases with temporal, not not stimulus regularity. Attention, Perception, \& Psychophysics, 77, 592-602. doi: 10.3758/s13414-014-0787-z

Horstmann, G. (2002). Evidence for attentional capture by a surprising color singleton in visual search. Psychological Science, 13(6), 499-505.

Hurewitz, F., Gelman, R., \& Schnitzer, B. (2006). Sometimes area counts more than number. Proceedings of the National Academy of Sciences USA, 103(51), 19599-19604.

Irwin, D. E., Colcombe, A. M., Kramer, A. F., \& Hahn, S. (2000). Attention and oculomotor capture by onset, luminance and color singletons. Vision Research, 40, 1443-1458.

Itti, L. (2007). Visual salience. Scholarpedia, 2(9), 3327.

Itti, L., \& Koch, C. (2001). Computational modelling of visual attention. Nature Reviews Neuroscience, 2, 194-203.

Ivry, R.B., \& Hazeltine, R.E. (1995). Perception and production of temporal intervals across a range of durations: Evidence for a common timing mechanism. Journal of Experimental Psychology: Human Perception and Performance, 21(1), 3-18.

James, W. (1890). The principles of psychology, Vol. I and II. New York: Henry Holt \& Co.

Jepma, M., Wagenmakers, E. J., \& Nieuwenhuis, S. (2012). Temporal expectation and information processing: A model-based analysis. Cognition, 122, 426-441. doi: 10.1016/j.cognition.2011.11.014

Javadi, A. H., \& Aichelburg, C. (2013). Training enhances the interference of numerosity on duration judgments. PLoS ONE, 8(1), e54098, 1-8. doi:10.1371/journal.pone.0054098

Jazayeri, M., \& Shadlen, M. N. (2010). Temporal context calibrates interval timing. Nature Neuroscience, 13, 1020-1028. doi: 10.1038/nn.2590

Jensen, O., Kaiser, J., \& Lachaux, J-P. (2007). Human gamma-frequency oscillations associated with attention and memory. Trends in Neurosciences, 30(7), 317-324. doi:10.1016/j.tins.2007.05.001

Johnston, A., Arnold, D. H., \& Nishida, S. (2006). Spatially localized distortions of event time. Current Biology, 16, 472-479. doi: 10.1016/j.cub.2006.01.032

Jones, L. A., Allely, C. S., \& Wearden, J. H. (2011). Click trains and the rate of information processing: Does "speeding up" subjective time make other psychological processes run faster? Quarterly Journal of Experimental Psychology, 62(2), 363-380. doi: 10.1080/17470218.2010.502580

Jones, L. A., \& Ogden, R. S. (2015). Vibrotactile timing: Are vibrotactile judgements of duration affected by repetitive stimulation? Quarterly Journal of Experimental Psychology, 12, 1-14. doi: 10.1080/17470218.2015.102735

Jones, L. A., Poliakoff, E., \& Wells, J. (2009). Good vibrations: Human interval timing in the vibrotactile modality. Quarterly Journal of Experimental Psychology, 61(11), 2171-2186. doi: $10.1080 / 17470210902782200$

Jones, L. A., \& Wearden, J. H. (2003). More is not necessarily better: Examining the nature of the temporal reference memory component in timing. Quarterly Journal of Experimental Psychology, 56B(4), 321-343. doi: 10.1080/02724990244000287

Jones, M. R. (1976). Time, our lost dimension: Toward a new theory of perception, attention, and memory. Psychological Review, 83, 323-355. doi: 10.1037/0033-295X.83.5.323

Jones, M. R. (2004). Attention and timing. In J. G. Neuhoff (Ed.), Ecological psychoacoustics (pp. 4985). San Diego, CA, USA: Elsevier, Inc.

Jones, M. R., \& Boltz, M. (1989). Dynamic attending and responses to time. Psychological Review, 96, 459-491. doi: 0.1037/0033-295X.96.3.459 
Jones, M.R., Boltz, M.G., \& Klein, J.M. (1993). Expected endings and judged duration. Memory \& Cognition, 21(5), 646-665.

Jones, M. R., Moynihan, H., MacKenzie, N., \& Puente, J. (2002). Temporal aspects of stimulus-driven attending in dynamic arrays. Psychological Science, 13, 313-319. doi: 10.1111/1467-9280.00458

Jonides, J. (1981). Voluntary versus automatic control over the mind's eye's movement. In J. B. Long \& A. Baddeley (Eds.). Attention and performance (Vol. IX, pp. 187-204). Hillsdale, NJ: Erlbaum.Kaliukhovich,

D. A., \& Vogels, R. (2011). Stimulus repetition probability does not affect repetition suppression in macaque inferior temporal cortex. Cerebral Cortex, 21(7), 1547-1558. doi: 10.1093/cercor/bhq207

Kanai, R., Paffen, C.L.E., Hogendoorn, H., \& Verstraten, F. A. J. (2006). Time dilation in dynamic visual display. Journal of Vision, 6, 1421-1430. doi: 10.1167/6.12.8

Kaneko, S., \& Murakami, I. (2009). Perceived duration of visual motion increases with speed. Journal of Vision, 9(7). Article 14. 1-12.

Kanwisher, N., \& Wojciulik, E. (2000). Visual attention: Insights from brain imaging. Nature Reviews Neuroscience, 1, 91-100. doi:10.1038/35039043

Kersten, D., Mamssian, P., \& Yuille, A. (2004). Object perception as Bayesian inference. Annual Review of Psychology, 55, 271-304. doi 10.1146/annurev.psych.55.090902.142005

Kim, E., \& McAuley, J. D. (2013). Effects of pitch distance and likelihood on the perceived duration of deviant auditory events. Attention, Perception, \& Psychophysics, 75(7), 1547-1558. doi: 10.3758/s13414-013-0490-5

Klein, R. M., \& Ivanoff, J. (2008). Inhibition of return. Scholarpedia, 3(10), 3650. doi: 10.4249 /scholarpedia.3650

Kliegl, K. M., \& Huckauf, A. (2014). Perceived duration decreases with increasing eccentricity. Acta Psychologica, 150, 136-145. doi: 10.1016/j.ctpsy.2014.05.007

Kliegl, K. M., Watrin, L., \& Huckhauf, A. (2015). Duration perception of emotional stimuli: Using evaluative conditioning to avoid sensory confounds. Cognition and Emotion, 29(8), 1350-1367. doi: 10.1080/02699931.2014.978841

Kovács, G., Kaiser, D., Kaliukhovic, D. A., Vidnyánszky, Z., \& Vogels, R. (2013). Repetition probability does not affect fMRI repetition suppression for objects. Journal of Neuroscience, 33(23), 98059812. doi: 10.1523/JNEUROSCI.3423-12.2013

Knudsen, E. I. (2007). Fundamental components of attention. Annual Review of Neuroscience, 30, 5778. doi: 10.1146/annurev.neuro.30.051606.094256

Kristofferson, A. B. (1980). A quantal step function in duration discrimination. Perception \& Psychophysics, 27, 300-306.

Kristofferson, A. B. (1984). Quantal and deterministic timing in human duration discrimination. Annals of the New York Academy of Sciences, 423, 3-15. doi: 10.1111/j.17496632.1984.tb23413.x

Krumhuber, E. G., Kappas, A., \& Manstead, A. S. R. (2013). Effects of dynamic aspects of facial expressions: A review. Emotion Review, 5, 41-46. doi: 10.1177/1754073912451349

Lake, J. I., LaBar, K. S., \& Meck, W. H. (in press). Emotional modulation of timing and time perception. Neuroscience and Biobehavioral Reviews.

Lake, J. I., \& Meck, W. H. (2013). Differential effects of amphetamine and haloperidol on temporal reproduction: Dopaminergic regulation of attention and clock speed. Neuropsychologia, 51, 284-292. doi: 10.1016/j.neuropsychologia.2012.09.014 
Laming, D. (1997). The Measurement of Sensation. Oxford: Oxford University Press.

Lander, K., \& Bruce, V. (2003). The role of motion in learning new faces. Visual Cognition, 10, 897912.

Large, E. W., \& Jones, M. R. (1999). The dynamics of attending: How people track time-varying events. Psychological Review, 106, 119-159. doi: 10.1037//0033-295X.106.1.119

Latimer, K., Curran, W., \& Benton, C. P. (2014). Direction-contingent duration compression is primarily retinotopic. Vision Research, 105, 47-52. doi: 10.1016/j.visres.2014.09.004

Lee, H., Simpson, G. V., Logothetis, N. K., \& Rainer, G. (2005). Phase locking of single neuron activity to theta oscillations during working memory in monkey extrastriate visual cortex. Neuron, 45, 147-156. doi:10.1016/j.neuron.2004.12.025

Lee, K-H., Seelam, K., \& O'Brien, T. (2011). The relativity of time percption produced by facial emotion stimuli. Cognition and Emotion, 25, 1471-1480

Lejeune, H. (1998). Switching or gating? The attentional challenge in cognitive models of psychological time. Behavioural Processes, 44, 127-145. doi:10.1016/S0376-6357(00)00141-8

Lejeune, H., \& Wearden, J. H. (2006). Scalar properties in animal timing: Conformity and violations. Quarterly Journal of Experimental Psychology, 59, 1875-1908. doi: 10.1080/17470210600784649

Leo, F., Romei, V., Freeman, E., Ladavas, E., \& Driver, J. (2011). Looking sounds enhance orientation sensitivity for visual stimuli on the same side as such sounds. Experimental Brain Research, 213, 193-201. doi: 10.1007/s00221-011-2742-8

Leopold, D. A., O’Toole, A. J., Vetter, T., \& Blanz, V. (2001). Prototype-reference shape encoding revealed by high-level aftereffects. Nature Neuroscience, 4(1), 89-94. doi: 10.1038/82947

Lewis, P.A., \& Miall, R.C. (2003). Brain activation patterns during measurement of sub- and suprasecond intervals. Neuropsychologia, 41(12), 1583-1592. doi: 10.1016/S0028-3932(03)00118-0

Lewis, P.A., \& Miall, R.C. (2009). The precision of temporal judgement: milliseconds, many minutes and beyond. Philosophical Transactions of the Royal Society B: Biological Sciences, 364(1525), 1897-1905. doi: 10.1098/rstb.2009.0020

Lhamon, W. T., \& Goldstone, S. (1975). Movement and the judged duration of visual targets. Bulletin of the Psychonomic Society, 5(1), 53-54. doi: 10.3758/BF03336701

Lin, J. Y., Franconeri, S., \& Enns, J. T. (2007). Objects on a collision path with the observer demand attention. Psychological Science, 19(7), 686-692. doi: 10.1111/j.1467-9280.2008.02143.x

Linares, D., \& Gorea, A. (2015). Temporal frequency of events rather than speed dilates perceived duration of moving objects. Scientific Reports, 5, Article 8825, 1-9. doi: 10.1038/srep08825

Lipp, O. V., \& Derakshan, N. (2005). Attentional bias to pictures of fear-relevant animals in a dot probe task. Emotion, 5(3), 365-369. doi: 10.1037/1528-3542.5.3.365

Lisman, J. E. \& Idiart, M. A. P. (1995). Storage of $7 \pm 2$ short-term memories in oscillatory subcycles. Science, 267, 1512-1515. doi:10.1126/science.7878473

Livesey, A. C., Wall, M. B., \& Smith, A. T. (2007). Time perception: Manipulation of task difficulty dissociates clock functions from other cognitive demands. Neuropsychologia, 45, 321-331. doi: 10.1016/j.neuropsychologia.2006.06.033

Lloyd, D., \& Arstila, V. (2014). Subjective time: The philosophy, psychology, and neuroscience of temporality, Cambridge, MA: MIT Press.

Loftus, G. R. (1985). Picture perception: Effects of luminance on available information and information-extraction rate. Journal of Experimental Psychology: General, 114(3), 342-356. doi: 10.1037/0096-3445.114.3.342 
Los, S. A., \& Horoufchin, H. (2011). Dissociative patterns of foreperiod effects in temporal discrimination and reaction time tasks. Quarterly Journal of Experimental Psychology, 64, 10091020. doi: $10.1080 / 17470218.2010 .532225$

Lourenco, S. F., \& Longo, M. R. (2010). General magnitude representation in human infants. Psychological Science, 21(6), 873-881. doi: 10.1177/0956797610370158

Lu, A., Hodges, B., Zhang, J., \& Zhang, J. X. (2009). Contextual effects on number-time interaction. Cognition, 113, 117-122. doi: 10.1016/j.cognition.2009.07.001

Lu, A., Mo, A., \& Hodges, B. H. (2011). The weight of time: Affordances for an integrated magnitude system. Journal of Experimental Psychology: Human Perception and Performance, 37(6), 18551866. doi: $10.1037 / \mathrm{a} 0024673$

Lui, M. A., Penney, T. B., \& Schirmer, A. (2011). Emotion effects on timing: Attention versus pacemaker accounts. PLOS ONE, 6(7), e21829, 1-9. doi: 10.1371/journal.pone.0021829.

Lustig, C. (2011). The neuroscience of time and number: Untying the Gordian knot. Frontiers in Integrative Neuroscience, 5:47. doi: 10.3389/fnint.2011.00047

Lustig, C., Matell, M. S., \& Meck, W. H. (2005). Not "just" a coincidence: Frontal-striatal interactions in working memory and interval timing. Memory, 13, 441-448. doi: 10.1080/09658210344000404

Lustig, C., \& Meck, W. H. (2011). Modality differences in timing and temporal memory throughout the lifespan. Brain and Cognition, 77, 298-303. doi: 10.1016/j.bandc.2011.07.007

Luzardo, A., Ludvig, E. A., \& Rivest, F. (2013). An adaptive drift-diffusion model of interval timing dynamics. Behavioural Processes, 95, 90-99. doi: 10.1016/j.beproc.2013.02.003

Macar, F. (2002). Expectancy, controlled attention and automatic attention in prospective temporal judgments. Acta Psychologica, 111, 243-262. doi:10.1016/S0001-6918(02)00052-5

Macar, F., Grondin, S., \& Casini, L. (1994). Controlled attention sharing influences time estimation. Memory \& Cognition, 22, 673-686.

Macar, F., Pouthas, V., \& Friedman, W. J. (Eds.). (1992). Time, action and cognition: Towards briding the gap. Series D: Behavioural and Social Sciences. Dordrecht, The Netherlands: Kluwer.

Macar, F., \& Vidal, F. (2009). Timing processes: An outline of behavioural and neural indicies not systematically considered in timing models. Canadian Journal of Experimental Psychology, 63, 227-239. doi: 10.1037/a0014457

MacDonald, C. J., Cheng, R. K., \& Meck, W. H. (2012). Acquisition of "Start" and "Stop" response thresholds in peak-interval timing is differentially sensitive to protein synthesis inhibition in the dorsal and ventral striatum. Frontiers in Integrative Neuroscience, 6, Article 10, 1-16. doi: 10.3389/fnint.2012.00010

Makin, A. D. J., Poliakoff, E., Dillon, J., Perrin, A., Mullet, T., \& Jones, L. A. (2012). The interaction between duration, velocity and repetitive auditory stimulation. Acta Psychologica, 139, 524531. doi:10.1016/j.actpsy.2012.01.013

Masson, M. E. J., \& Caldwell, J. I. (1998). Conceptually driven encoding episodes create perceptual misattributions. Acta Psychologica, 98, 183-210. doi:10.1016/S0001-6918(97)00042-5

Martinez-Conde, S., Macknik, S. L., \& Hubel, D. H. (2004). The role of fixational eye movements in visual perception. Nature Reviews Neuroscience, 5, 229-240. doi:10.1038/nrn1348

Matell, M. S., \& Meck, W. H. (2000). Neuropsychological mechanisms of interval timing behavior. Bioessays, 22, 94-103. doi: 10.1002/(SICI)1521-1878(200001)22:1<94::AID-BIES14>3.0.CO;2-E 
Matell, M. S., \& Meck, W. H. (2004). Cortico-striatal circuits and interval timing: Coincidencedetection of oscillatory processes. Cognitive Brain Research, 21, 139-170. doi:10.1016/j.cogbrainres.2004.06.012

Mattes, S., \& Ulrich, R. (1997). Response force is sensitive to the temporal uncertainty of response stimuli. Perception \& Psychophysics, 59, 1089-1097.

Mattes, S., \& Ulrich, R. (1998). Directed attention prolongs the perceived duration of a brief stimulus. Perception \& Psychophysics, 60, 1305-1317.

Matthews, W. J. (2015). Time perception: The surprising effects of surprising stimuli. Journal of Experimental Psychology: General, 144, 172-197. doi: 10.1037/xge0000041

Matthews, W. J. (2011a). Can we use verbal estimation to dissect the internal clock? Differentiating the effects of pacemaker rate, switch latencies, and judgment processes. Behavioural Processes, 86(1), 68-74. doi: 10.1016/j.beproc.2010.09.006

Matthews, W. J. (2011b). How do changes in speed affect the perception of duration? Journal of Experimental Psychology: Human Perception and Performance, 37, 1617-1627. doi: 10.1037/a0022193.

Matthews, W. J. (2011c). Stimulus repetition and the perception of time: The effects of prior exposure on temporal discrimination, judgment, and production. PLOS ONE, 6(5), e19815, 1-7. doi: 10.1371/journal.pone.0019815

Matthews, W. J. (2013). How does sequence structure affect the judgment of time? Exploring a weighted sum of segments model. Cognitive Psychology, 66, 259-282. doi: 10.1016/j.cogpsych.2013.01.001.

Matthews, W. J., Benjamin, C., \& Osborne, C. (2007). Memory for moving and static images. Psychonomic Bulletin \& Review, 14, 989-993

Matthews, W. J., \& Grondin, S. (2012). On the replication of Kristofferson's (1980) quantal timing for duration discrimination: Some learning but no quanta and not much os a Weber constant. Attention, Perception \& Psychophysics, 74(5), 1056-1072. doi: 10.3758/s13414-012-0282-3

Matthews, W. J., \& Meck, W. H. (2014). Time perception: The bad news and the good. Wiley Interdisciplinary Reviews: Cognitive Science, 5, 429-446. doi: 10.1002/wcs.1298

Matthews, W.J., \& Stewart, N. (2009a). The effects of interstimulus interval on sequential effects in absolute identification. Quarterly Journal of Experimental Psychology, 62, 2014-2029. doi: 10.1080/17470210802649285

Matthews, W. J., \& Stewart, N. (2009b). Psychophysics and the judgment of price: Judging complex objects on a non-physical dimension elicits sequential effects like those in perceptual tasks. Judgment and Decision Making, 4, 64-81.

Matthews, W. J., Stewart, N., \& Wearden, J. H (2011). Stimulus intensity and the perception of duration. Journal of Experimental Psychology: Human Perception and Performance, 37, 303-313. doi: $10.1037 / \mathrm{a} 0019961$.

Matthews, W. J., Terhune, D. B., van Rijn, H., Eagleman, D. M., Sommer, M. A., \& Meck, W. H. (2014). Subjective duration as a signature of coding efficiency: Emerging links among stimulus repetition, prediction coding, and cortical GABA levels. Timing \& Time Perception Reviews, 1(5), 1-11. doi: 10.1163/24054496_001_01_s005

Mauk, M. D., \& Buonomano, D. V. (2004). The neural basis of temporal processing. Annual Review of Neuroscience, 27, 307-340. doi: 10.1146/annurev.neuro.27.070203.144247 
Mayrhauser, L., Bergmann, J., Crone, J., \& Kronbichler, M. (2014). Neural repetition suppression: evidence for perceptual expectation in object-selective regions. Frontiers in Human Neuroscience, 8, Article 225, 1-8. doi: 10.3389/fnhum.2014.00225

McAuley, J. D., \& Jones, M. R. (2003). Modeling effects of rhythmic context on perceived duration: A comparison of interval and entrainment approaches. Journal of Experimental Psychology: Human Perception and Performance, 29(6), 1102-1125. doi: 10.1037/0096-1523.29.6.1102

McAuley, J. D., \& Fromboluti, E. K. (2014). Attentional entrainment and perceived event duration. Philosophical Transactions of the Royal Society B, 369, 20130401. doi: 10.1098/rstb.2013.0401

McAuley, J. D., \& Kidd, G. R. (1998). Effect of deviations from temporal expectations on tempo discrimination of isochronous tone sequences. Journal of Experimental Psychology: Human Perception and Performance, 24(6), 1786-1800.

McCormack, T. (2015). The development of temporal cognition. In: Handbook of Child Psychology and Developmental Science ( $7^{\text {th }}$ Ed.), R.M.Lerner (Ed.) Wiley.

McDonald, J. J., Green, J. J., Störmer, V. S., \& Hillyard, S. A. (2012). Cross-modal spatial cuing of attention influences visual perception. In M.M. Murray \& M.T. Wallace (Eds.) The neural bases of multisensory processes. Boca Raton: CRC Press.

Meck, W. H., 1984. Attentional bias between modalities: Effects on the internal clock, memory and decision stages used in animal time discrimination. Annals of the New York Academy of Sciences, 421, 528-541. doi: 10.1111/j.1749-6632.1984.tb23457.x

Meck, W. H., \& Church, R. M. (1983). A mode control model of counting and timing processes. Journal of Experimental Psychology: Animal Behavior Processes, 9, 320-334. doi: 10.1037/00977403.9.3.320

Meck, W. H., Doyère, V., \& Gruart, A. (2012). Interval timing and time-based decision making. Frontiers in Integrative Neuroscience, 6:13. doi: 10.3389/fnint.2012.00013

Meck, W. H., \& Ivry, R. B. (in press). Interval timing and skill learning: The multisensory representation of time and action. Current Opinion in Behavioral Sciences.

Meck, W. H., \& Malapani, C. (2004). Neuroimaging of interval timing. Cognitive Brain Research, 21, 133-137. doi:10.1016/j.cogbrainres.2004.07.010

Meck, W. H., Penney, T. B., \& Pouthas, V. (2008). Cortico-striatal representation of time in animals and humans. Current Opinion in Neurobiology, 18, 145-152. doi: 10.1016/j.conb.2008.08.002.

Melgire, M., Ragot, R., Samson, S., Penney, T. B., Meck, W. H., \& Pouthas, V. (2005). Auditory/visual duration bisection in patients with left or right medial-temporal lobe resection. Brain and Cognition, 58, 119-124. doi: 10.1016/j.bandc.2004.09.013

Merchant, H., Harrington, D. L., \& Meck, W. H. (2013). Neural basis of the perception and estimation of time. Annual Review of Neuroscience, 36, 313-336. doi: 10.1146/annurev-neuro-062012170349

Merchant, H., Zarco, W., Pérez, O., Prado, L., \& Bartolo, R. (2011). Measuring time with different neural chronometers during a synchronization-continuation task. Proceedings of the National Academy of Sciences, USA, 108, 19784-19789. doi: 10.1073/pnas.1112933108.

Merchant, H., Zarco, W., \& Prado, L. (2008). Do we have a common mechanism for measuring time in the hundreds of millisecond range? Evidence from multiple-interval timing tasks. Journal of Neurophysiology, 99, 939-949. doi: 10.1152/jn.01225.2007

Mella, N., Conty, L., \& Pouthas, V. (2011). The role of physiological arousal in time perception: Psychophysiological evidence from an emotion regulation paradigm. Brain and Cognition, 75, 182-187. doi: 10.1016/j.bandc.2010.11.012 
Meyer, W-U., Niepel, M., Rudolph, U., Schützwohl, A. (1991). An experimental analysis of surprise. Cognition and Emotion, 5, 295-311.

Miall, C. (1989). The storage of time intervals using oscillating neurons. Neural Computation, 1, 359371.

Michon, J. A. (1972). Processing of temporal information and the cognitive theory of time experience. In J.T. Frasier, F.C. Haber, \& G.H. Muller (Eds.), The study of time. (pp. 242-258). New York: Springer-Verlag.

Miki, A., \& Santi, A. (2005). The perception of empty and filled time intervals by pigeons. Quarterly Journal of Experimental Psychology 58A, 31-45. doi: 10.1080/0272499044000032

Miller, J. E., Carlson, L. A., \& McAuley, J. D. (2013). When what you hear influences when you see: Listening to an auditory rhythm influences the temporal allocation of visual attention. Psychological Science, 24(1), 11-18. doi: 10.1177/0956797612446707

Miniussi, C., Wilding, E. L., Coull, J. T., \& Nobre, A. C. (1999). Orienting attention in time: Modulation of brain potentials. Brain, 122, 1507-1518. doi: 10.1093/brain/122.8.1507

Mioni, G., Stablum, F., McClintock, S. M., \& Grondin, S. (2014). Different methods for reproducing time, different results. Attention, Perception, \& Psychophysics, 76, 675-681. doi: 10.3758/s13414-014-0625-3

Moore, B. C. J. (2012). An introduction to the psychology of hearing. $6^{\text {th }}$ Ed. Bingley: Emerald.

Moreton, B. J., \& Ward, G. (2010). Time scale similarity and long-term memory for autobiographical events. Psychonomic Bulletin \& Review, 17, 510-515.

Morgan, M. J., Watamaniuk, S. N. J., \& McKee, S. P. (2000). The use of an implicit standard for measuring discrimination thresholds. Vision Research, 40, 2341-2349.

Morrone, M. C., Cicchini, M., \& Burr, D. (2010). Spatial maps for time and motion. Experimental Brain Research, 206, 121-128. doi: 10.1007/s00221-010-2334-z

Müller-Gethmann, H., Ulrich, R., \& Rinkenauer, G. (2003). Locus of the effect of temporal preparation: Evidence from the lateralized readiness potential. Psychophysiology, 40, 597-611.

Murray, S. O., Boyaci, H., \& Kersten, D. (2006). The representation of perceived angular size in human primary visual cortex. Nature Neuroscience, 9(3), 429-434.

Myers, G. E. (1971). Williams James on time perception. Philosophy of Science, 38, 353-360.

New, J. J., \& Scholl, B. J. (2009). Subjective time dilation: Spatially local, object-based, or a global visual experience? Journal of Vision, 9(2), Article 4, 1-11. doi: 10.1167/9.2.4

Nobre, K., \& Coull, J. (2010). Attention and time. Oxford: Oxford University Press.

Noguchi, Y., \& Kakigi, R. (2006) Time representations can be made from non-temporal information in the brain: An MEG study. Cerebral Cortex, 16, 1797-1808. doi: 10.1093/cercor/bhj117

Noulhiane, M., Mella, N., Samson, S., Ragot, R., \& Pouthas, V. (2007). How emotional auditory stimuli modulate time perception. Emotion, 7(4), 697-704. doi: 10.1037/1528-3542.7.4.697

Ogden, R. S., Wearden, J. H., \& Jones, L. A. (2008). The remembrance of times past: Interference in temporal reference memory. Journal of Experimental Psychology: Human Perception and Performance, 34, 1524-1544. doi: 10.1037/a0010347.

Öhman, A., Flykt, A., \& Esteves, F. (2001). Emotion drives attention: Detecting the snake in the grass. Journal of Experimental Psychology: General, 130(3), 466-478.

Oldoni, D., De Coensel, B., Boes, M., Rademaker, M., De Baets, B., Van Venterghem, T., \& Botteldooren, D. (2013). A computational model of auditory attention for use in soundscape research. Journal of the Acoustical Society of America, 134(1), 852-861. doi: 10.1121/1.4807798 
Oliveri, M., Vicario, C.M., Salerno, S., Koch, G., Turriziani, P., Mangano, R., ... Caltagirone, C. (2008). Perceiving numbers alters time perception. Neuroscience Letters, 438, 308-311. doi: 10.1016/j.neulet.2008.04.051

Ono, F., \& Kawahara, J. (2005). The effect of unconscious priming on temporal production. Consciousness and Cognition, 14, 474-482. doi:10.1016/j.concog.2005.02.001

Ono, F., \& Kawahara, J. I. (2007). The subjective size of visual stimuli affects the perceived duration of their presentation. Perception \& Psychophysics, 69, 952-957.

Ono, F., \& Kawahara, J. I. (2008). The effect of false memory on temporal perception. Psychological Research, 72, 61-64.

Ono, F., \& Kitazawa, S. (2010a). The effect of perceived motion-in-depth on time perception. Cognition, 115, 140-146. doi: 10.1016/j.cognition.2009.12.006

Ono, F., \& Kitazawa, S. (2010b). Shortening of subjective tone intervals followed by repetitive tone stimuli. Attention, Perception, \& Psychophysics, 72, 492-500.

Ono, F., \& Kitazawa, S. (2011). Shortening of subjective visual intervals followed by repetitive stimulation. PLOS ONE, 6(12), e28722, 1-6. doi: 10.1371/journal.pone.0028722

Ono, F., Yamada, K., Chujo, K., \& Kawahara, J. I. (2007). Feature-based attention influences later temporal perception. Perception \& Psychophysics, 69, 544-549.

Oprisan, S. A., \& Buhusi, C. V. (2011). Modeling pharmacological clock and memory patterns of interval timing in a striatal beat-frequency model with realistic, noisy neurons. Frontiers in Integrative Neuroscience, 5:52. doi: 10.3389/fnint.2011.00052

Oprisan, S. A., \& Buhusi, C. V. (2014). What is all the noise about in interval timing? Philosophical Transactions of the Royal Society B, 369. doi:10.1098/rstb.2012.0459

Orgs, G., Bestmann, S., Schuur, F., \& Haggard, P. (2011). From body form to biological motion: The apparent velocity of human movement biases subjective time. Psychological Science, 22(6), 712-717. doi: 10.1177/0956797611406446

Most, S. B., Chun, M. M., Widders, D. M., \& Zald, D. H. (2005). Attentional rubbernecking: Cognitive control and personality in emotion-induced blindness. Psychonomic Bulletin \& Review, 12(4), 654-661.

Paller, K. A., Mayes, A. R., McDermott, M., Pickering, A. D., \& Meudell, P. R. (1991). Indirect measures of memory in a duration-judgement task are normal in amnesic patients. Neuropsychologia, 10, 1007-1018.

Palumbo, L., Ogden, R., Makin, A. D. J., \& Bertamini, M. (2014). Examining visual complexity and its influence on perceived duration. Journal of Vision, 14(14), Article 3, 1-18. doi: 10.1167/14.14.3

Pan, Y., \& Luo, Q-Y. (2012). Working memory modulates the perception of time. Psychonomic Bulletin \& Review, 19, 46-51. doi: 10.3758/s13423-011-0188-4

Parducci, A. (1965). Category judgment: A range-frequency model. Psychological Review, 72, 407418.

Pariyadath, V., \& Eagleman, D. (2007). The effect of predictability on subjective duration. PLOS ONE, 2(11). e1264, 1-6.. doi: 10.1371/journal.pone.0001264

Pariyadath, V., \& Eagleman, D. M. (2008). Brief subjective durations contract with repetition. Journal of Vision, 8, 11-16.

Pariyadath, V., \& Eagleman, D. M. (2012). Subjective duration distortions mirror neural repetition suppression. PLOS ONE, 7(12). e49362, 1-4. doi: 10.1371/journal.pone.0049362

Pavlik, P. I., \& Anderson, J. R. (2005). Practice and forgetting effects on vocabulary memory: An activation-based model of the spacing effect. Cognitive Science, 29, 559-586. 
Penney, T. B., Brown, G. D. A., \& Wong, J. K. L. (2014). Stimulus spacing effects in duration perception are larger for auditory stimuli: Data and a model. Acta Psychologica, 147, 97-104. 10.1016/j.actpsy.2013.07.017

Penney, T. B., Gibbon, J., \& Meck, W. H. (2000). Differential effects of auditory and visual signals on clock speed and temporal memory. Journal of Experimental Psychology: Human Perception and Performance, 26, 1770-1787. doi: 10.1016/j.bandc.2004.09.012

Penney, T. B., Gibbon, J., \& Meck, W. H. (2008). Categorical scaling of duration bisection in pigeons (Columba livia), mice (Mus musculus), and humans (Homo sapiens). Psychological Science, 19, 1103-1109. doi: 10.1111/j.1467-9280.2008.02210.x.

Penney, T. B., Meck, W. H., Roberts, S. A., Gibbon, J., \& Erlenmeyer-Kimling, L. (2005). Interval-timing deficits in individuals at high risk for schizophrenia. Brain and Cognition, 58, 109-118. doi:10.1016/j.bandc.2004.09.012

Penton-Voak, I. S., Edwards, H., Percival, A., \& Wearden, J. H. (1996). Speeding up an internal clock in humans? Effects of click trains on subjective duration. Journal of Experimental Psychology: Animal Behavior Processes, 22, 307-320.

Petrov, A. \& Anderson, J. R. (2005). The dynamics of scaling: A memory-based anchor model of category rating and absolute identification. Psychological Review, 112(2), 383-416.

Pinel, P., Piazza, M., Le Bihan, D., \& Dehaene, S. (2004). Distributed and overlapping cerebral representations of number, size, and luminance during comparative judgments. Neuron, 41(6), 983-993. doi: 10.1016/S0896-6273(04)00107-2

Piras, F., \& Coull, J. T. (2011). Implicit, predictive timing draws upon the same scalar representation of time as explicit timing. PLOS ONE, 6(3), e18203, 1- 11. doi: 10.1371/journal.pone.001820

Plourde, M., Gamache, P-L., \& Grondin, S. (2008). Filled intervals are perceived as longer than empty ones: The effect occurs even with a between-session design. Proceedings of Fechner Day, 24, 87-92.

Pöppel, E. (2009). Pre-semantically defined temporal windows for cognitive processing. Philosophical Transactions of the Royal Society, Biological Sciences, 364, 1887-1896. doi: 10.1098/rstb.2009.0015

Posner, M. I. (1980). Orienting of attention. Quarterly Journal of Experimental Psychology, 32, 3-25.

Posner, M. I. and Y. Cohen (1984). Components of visual orienting. Attention and Performance X: Control of Language Processes. H. Bouma and D. Bonwhuis. Hillsdale, N. J., Erlbaum: 551-556.

Pouthas, V., George, N., Poline, J-B., Pfeuty, M., VandeMoorteele, P-F., Hugueville, L., ...Renault, B. (2005). Neural network involved in time perception: An fMRI study comparing long and short interval estimation. Human Brain Mapping, 25, 433-441. doi: 10.1002/hbm.20126

Poynter, W. D., (1983). Duration judgment and the segmentation of experience. Memory \& Cognition, 11, 77-82.

Poynter, W. D. (1989). Judging the duration of time intervals: A process of remembering segments of experience. In I. Levin \& D. Zakay (Eds.), Time and human cognition: A life-span perspective (pp. 305-331). Amsterdam: North Holland.

Proulx, M. J. (2010). Size matters: Large objects capture attention in visual search. PLOS ONE, 5(12), e15293, 1-6. doi: 10.1371/journal.pone.0015293

Proulx, M. J., \& Green, M. (2011). Does apparent size capture attention in visual search? Evidence from the Müller-Lyer illusion. Journal of Vision, 11(13), Article 21, 1-6. doi: 10.1167/11.13.21

Rammsayer, T.H. (1999). Neuropharmacological evidence for different timing mechanisms in humans. Quarterly Journal of Experimental Psychology, 52B(3), 273-286. 
Rammsayer, T.H., \& Lima, S.D. (1991). Duration discrimination of filled and empty auditory intervals: Cognitive and perceptual factors. Perception \& Psychophysics, 50(6), 565-574.

Rammsayer, T.H., \& Troche, S.J. (2014). In search of the internal structure of the processes underlying interval timing in the sub-second and second range: A confirmatory factor analysis approach. Acta Psychologica, 147, 68-74. doi: 10.1016/j.actpsy.2013.05.004

Rammsayer, T., \& Ulrich, R. (2001). Counting models of temporal discrimination. Perception \& Psychophysics, 8(2), 270-277.

Rammsayer, T.H., \& Ulrich, R. (2001). No evidence for qualitative differences in the processing of short and long temporal intervals. Acta Psychologica, 120(2), 141-171. doi: 10.1016/j.actpsy.2005.03.005

Rammsayer, T. H., \& Verner, M. (2014). The effect of nontemporal stimulus size on perceived duration as assessed by the method of reproduction. Journal of Vision, 14(50), Article 17, 1-10. doi: 10.1167/14.5.17

Rakitin, B. C., Gibbon, J., Penney, T. B., Malapani, C., Hinton, S. C., \& Meck, W. H. (1998). Scalar expectancy theory and peak-interval timing in humans. Journal of Experimental Psychology: Animal Behavior Processes, 24, 15-33. doi: 10.1037/0097-7403.24.1.15

Rao, R. P. N., \& Ballard, D. H. (1999). Predictive coding in the visual cortex: A functional interpretation of some extra-classical receptive-field effects. Nature Neuroscience, 2, 79-87. doi: $10.1038 / 4580$

Ratcliff, R. (1978). A theory of memory retrieval. Psychological Review, 85, 59-108.

Ratcliff, R., Gomez, P., \& McKoon, G. (2004). A diffusion model accoun of the lexical decision task. Psychological Review, 111(1), 159-182. doi: 10.1037/0033-295X.111.1.159

Ratcliff, R., \& McKoon, G. (2008). The diffusion decision model: Theory and data for two-choice decision tasks. Neural Computation, 20, 873-922.

Ratcliff, R., \& Rouder, J. N. (1998). Modeling response times for two-choice decisions. Psychological Science, 9(5), 347-356.

Ratcliff, R., \& Smith, P. L. (2004). A comparison of sequential sampling models for two-choice reaction time. Psychological Review, 111(2), 333-367. doi: 10.1037/0033-295X.111.2.333

Rattat, A-C., \& Droit-Volet, S. (2012). What is the best and easiest method of prevening counting in different temporal tasks? Behavior Research Methods, 44, 67-80. doi: 10.3758/s13428-0110135-3

Reber, R., Zimmermann, T. D., \& Wurtz, P. (2004). Judgments of duration, figure-ground contrast, and size for words and nonwords. Perception \& Psychophysics, 66, 1105-1114.

Reingold, E. M., \& Merikle, P. M. (1988). Using direct and indirect measures to study perception without awareness. Perception \& Psychophysics, 44, 563-575.

Repp, B. H., Mendlowitz, H. B., \& Hove, M. J. (2013). Does rapid auditory stimulation accelerate an internal pacemaker? Don't bet on it. Timing \& Time Perception, 1, 65-76. doi: $10.1163 / 22134468-00002001$

Rhodes, G., Watson, T. L., Jeffery, L., \& Clifford, C. W. G. (2010). Perceptual adaptation helps us identify faces. Vision Research, 50, 963-968. doi: 10.1016/j.visres.2010.03.003

Rivest, F., \& Benjio, Y. (2011). Adaptive drift-diffusion process to learn time intervals. arXiv, 1103.2382v1

Rohenkohl, G., Coull, J. T., \& Nobre, A. C. (2011). Behavioral dissociation between exogenous and endogenous temporal orienting of attention. PLOS ONE, 6(1), e14620, 1-5. doi:

10.1371/journal.pone.0014620 
Rohenkohl, G., Cravo, A. M., Wyart, V., \& Nobre, A. C. (2012). Temporal expectation improves the quality of sensory information. Journal of Neuroscience, 32, 8424-8428. doi: 10.1523/JNEUROSCI.0804-12.2012.

Rohenkohl, G., Gould, I.C., Pessoa, J., \& Nobre, A.C. (2014). Combining spatial and temporal expectations to improve visual perception. Journal of Vision, 14(4), Article 8, 1-13. doi: $10.1167 / 14.4 .8$

Roitman, J. D., Brannon, E. M., \& Platt, M. L. (2007). Monotonic coding of numerosity in macaque lateral intraparietal area. PLoS Biology, 5(8), e208, 1672-1682. doi: 10.1371/journal.pbio.0050208

Rolke, B. (2008). Temporal preparation facilitates perceptual identification of letters. Perception \& Psychophysics, 70, 1305-1313.

Rolke, B., \& Hoffman, P. (2007). Temporal uncertainty degrades perceptual processing. Psychonomic Bulletin \& Review, 14, 522-526.

Rolke, B., Ulrich, R., \& Bausenhart, K. M. (2006). Attention delays perceived stimulus offset. Vision Research, 46, 2926-2933.

Rose, D., \& Summers, J. (1995). Duration illusions in a train of visual stimuli. Perception, 24(10), 1177-1187. doi: 10.1068/P241177

Rousseau, R., Picard, D., \& Pitre, E. (1984). An adaptive counter model for time estimation. Annals of the New York Academy of Sciences, 423, 639-642.

Roux, F., \& Uhlhaas, P. J. (2014). Working memory and neural oscialltions: alpha-gamma versus theta-gamme codes for distinct WM information? Trends in Cognitive Sciences, 18(1), 16-25.

Ruz, M., \& Lupiáñez, J. (2002). A review of attentional capture: On its automaticity and sensitivity to endogenous control. Psicológica, 23, 283-309.

Sadeghi, N. G., Pariyadath, V., Apte, S., Eagleman, D. M., \& Cook, E. P. (2011). Neural correlates of subsecond time distortion in the middle temporal area of visual cortex. Journal of Cognitive Neuroscience, 23(12), 3829-3840. doi: 10.1162/jocn_a_00071

Schiffman, H. R., \& Bobko, D. J. (1974). Effects of stimulus complexity on the perception of brief temporal intervals. Journal of Experimental Psychology, 103(1), 156-159.

Schindel, R., Rowlands, J., \& Arnold, D. H. (2011). The oddball effect: Perceived duration and predictive coding. Journal of Vision, 11(2), 17, 1-9. doi: 10.1167/11.2.17

Seibold, V. C., Bausenhart, K. M., Rolke, B., \& Ulrich, R. (2011). Does temporal preparation increase the rate of sensory information accumulation? Acta Psychologica, 137, 56-64. doi: 10.1016/j.actpsy.2011.02.006

Seifried, T., \& Ulrich, R. (2010). Does the asymmetry effect inflate the temporal expansion of odd stimuli? Psychological Research, 74(1), 90-98. doi: 10.1007/s00426-008-0187-x

Seifried, T., \& Ulrich, R. (2011). Exogenous visual attention prolongs perceived duration. Attention, Perception, \& Psychophysics, 73, 68-85.

Shallice, T. (1964). The detection of change and the perceptual moment hypothesis. British Journal of Mathematical and Statistical Psychology, 17, 113-135. doi: 10.1111/j.2044-

8317.1964.tb00254.x

Shi, Z., Church, R. M., \& Meck, W. H. (2013). Bayesian optimization of time perception. Trends in Cognitive Sciences, 17, 556-564. doi: 10.1016/j.tics.2013.09.009

Shi, Z., Lia, L., \& Müller, H. J. (2012). Modulation of tactile duration judgments by emotional pictures. Frontiers in Integrative Neuroscience, 6, Article 24, 1-9. doi: 10.3389/fnint.2012.00024 
Shibasaki, M., \& Masataka, N. (2014). The color red distorts time perception for men, but not for women. Scientific Reports, 4, Article 5899, 1-4. doi:10.1038/srep05899

Simen, P., Balci, F., deSouza, L., Cohen, J. D., \& Holmes, P. (2011a). A model of interval timing by neural integration. Journal of Neuroscience, 31, 9238-9253.

Simen, P., Balci, F., deSouza, L., Cohen, J. D., \& Holmes, P. (2011b). Interval timing by long-range temporal integration. Frontiers in Integrative Neuroscience, 5, Article 28, 1-2, doi: $10.3389 /$ fnint.2011.00028

Simen, P., Rivest, F., Ludvig, E. A., Balci, F., \& Killeen, P. (2013). Timescale invariance in the pacemaker-accumulator family of timing models. Timing \& Time Perception, 1, 159-188. doi: 10.1163/22134468-00002018

Skarratt, P. A., Cole, G. G., \& Gellatly, A. R. H (2009). Prioritization of looming and receding objects: Equal slopes, different intercepts. Attention, Perception, \& Psychophysics, 71(4), 964-970. doi: 10.3758/APP.71.4.964

Soto, D., Humphreys, G. W., \& Rotshtein, P. (2007). Dissociating neural mechanisms of memorybased guidance of visual selection. Proceedings of the National Academy of Sciences, 104(43), 17186-17191. doi: 10.1073_pnas.0703706104

Sperling, G. (1960). The information available in brief visual presentations. Psychological Monographs: General and Applied, 74(11), 1-29.

Spurgeon, J., Ward, G., \& Matthews, W.J. (2014). Why do participants initiate free recall of short lists of words with the first list item? Toward a general episodic memory explanation. Journal of Experimental Psychology: Learning, Memory, and Cognition, 40(6), 1551-1567. doi: $10.1037 / \mathrm{xlm} 0000028$

Stevens, S. S. (1957). On the psychophysical law. Psychological Review, 64, 153-181.

Stewart, N., Brown, G. D. A., \& Chater, N. (2005). Absolute identification by relative judgment. Psychological Review, 112, 881-911.

Summerfield, C., Trittschuh, E. H., Monti, J. M., Mesulam, M. M., \& Egner, T. (2008). Neural repetition suppression reflects fulfilled perceptual expectations. Nature Neuroscience, 11, 10041006. doi: $10.1038 / \mathrm{Nn} .2163$

Summerfield, C., Wyart, V., Johnen, V. M., \& de Gardelle, V. (2011). Human scalp electropencephalography reveals that repetition suppression varies with expectation. Frontiers in Human Neuroscience, 5, Article 67, 1-13. doi: 10.3389/fnhum.2011.00067

Sutherland, C. A. M., Thut, G., \& Romei, V. (2014). Hearing brighter: Changing in-depth visual perception through looming sounds. Cognition, 132, 312-323. doi: 10.1016/j.cognition.2014.04.011

Taatgen, N., \& van Rijn, H. (2011). Traces of times past: Representations of temporal intervals in memory. Memory \& Cognition, 39, 1546-1560. doi: 10.3758/s13421-011-0113-0.

Taatgen, N. A., van Rijn, H., \& Anderson, J. (2007). An integrated theory of prospective time interval estimation: The role of cognition, attention, and learning. Psychological Review, 114, 577-598. doi: 10.1037/0033-295X.114.3.577

Tallon-Baudry, C., \& Bertrand, O. (1999). Oscillatory gamma activity in humans and its role in object representation. Trends in Cognitive Sciences, 3(4), 151-162. doi:10.1016/S1364-6613(99)012991

Teki, S., Grube, M., \& Griffiths, T. D. (2012). A unified model of time perception accounts for duration-based and beat-based timing mechanisms. Frontiers in Integrative Neuroscience, 5:90. doi: 10.3389/fnint.2011.00090 
Tenenbaum, J. B., Kemp, C., Griffiths, T. L., \& Goodman, N. D. (2011). How to grow a mind: Statistics, structure, and abstraction. Science, 331, 1279-1285. doi: 10.1126/science.1192788.

Theeuwes, J. (2010). Top-down and bottom-up control of visual selection. Acta Psychologica, 135, 77-99. doi: 10.1016/j.actpsy.2010.02.006

Thomas, E. A. C., \& Cantor, N. E. (1975). Duality of simultaneous time and size perception. Perception \& Psychophysics, 18, 44-48.

Thomas, E. A. C., \& Weaver, W. B. (1975). Cognitive processing and time perception. Perception \& Psychophysics, 17, 363-367.

Thompson, P., \& Burr, D. (2009). Visual aftereffects. Current Biology, 19, R11-R14. doi: 10.1016/j.cub.2008.10.014

Tipples, J. (2008). Negative emotionality influences the effects of emotion on time perception. Emotion, 8(1), 127-131. doi: 10.1037/1528-3542.8.1.127

Tipples, J. (2011). When time stands still: Fear-specific modulation of temporal bias due to threat. Emotion, 11(1), 74-80. doi: 10.1037/a0022015

Todd, R. M., Talmi, D., Schmitz, T. W., Susskind, J., \& Anderson, A. K. (2012). Psychophysical and neural evidence for emotion-enhanced perceptual vividness. Journal of Neuroscience, 32(33), 11201-11212. doi: 10.1523/JNEUROSCI.0155-12.2012

Todorović, D. (2010). Context effects in visual perception and their explanations. Review of Psychology, 17(1), 17-32.

Treisman, M. (2013). The information-processing model of timing (Treisman 1963): Its sources and further development. Timing \& Time Perception, 1, 131-158. doi: 10.1163/22134468-00002017

Treisman, M., Faulkner, A., Naish, P. L. N., \& Brogan, D. (1990). The internal clock: Evidence for a temporal oscillator underlying time perception with some estimates of its characteristic frequency. Perception, 19, 705-743.

Tremblay, S., \& Fortin, C. (2003). Break expectancy in duration discrimination. Journal of Experimental Psychology: Human Perception and Performance, 29(4), 823-831. doi: 10.1037/0096-1523.29.4.823

Tse, P. U., Intriligator, J., Rivest, J., \& Cavanagh, P. (2004). Attention and the subjective expansion of time. Perception \& Psychophysics, 66, 1171-1189.

Tucci, V., Buhusi, C. V., Gallistel, C. R. \& Meck, W. H. (2014). Towards an integrated understanding of the biology of timing. Philosophical Transactions of the Royal Society - London B, 369, 20120470. doi: 10.1098/rstb.2012.0470

Tversky, B., \& Sherman, T. (1975). Picture memory improves with longer on time and off time. Journal of Experimental Psychology: Human Learning and Memory, 104(2), 114-118.

Ulrich, R., Nitschke, J., \& Rammsayer, T. (2006). Perceived duration of expected and unexpected stimuli. Psychological Research, 70, 77-87.

Vangkilde, S., Coull, J. T., \& Bundesen, C. (2012). Great expectations: Temporal expectation modulates perceptual processing speed. Journal of Experimental Psychology: Human Perception and Performance, 38, 1183-1191. doi: 10.1037/a0026343

van Rijn, H., Gu, B-M., \& Meck, W. H. (2014). Dedicated clock/timing-circuit theories of time perception and timed performance. Advances in Experimental Medicine and Biology, 829, 7599. doi: 10.1007/978-1-4939-1782_5

van Rijn, H., Kononowicz, T. W., Meck, W. H., Ng, K. K., \& Penney, T. B. (2011). Contingent negative variation and its relation to time estimation: A theoretical evaluation. Frontiers in Integrative Neuroscience, 5:91. doi: 10.3389/fnint.2011.00091 
van Rijn, H., \& Taatgen, N. A. (2008). Timing of multiple overlapping intervals: How many clocks do we have? Acta Psychologica, 129, 365-375. doi: 10.1016/j.actpsy.2008.09.002

van Wassenhove, V., Buonomano, D. V., Shimojo, S., \& Shams, L. (2008). Distortions of subjective time perception within and across senses. PLOS ONE, 3(1), e1437, 1-13. doi: 10.1371/journal.pone.0001437

Vatakis A., \& Allman M. J. (Eds.) (2015). Time distortions in mind: Temporal processing in clinical populations. Boston: Brill Academic Publishers.

Vatakis, A., Esposito, A., Giagkou, M., Cummins, F., \& Papadelis, G. (2011). Multidisciplinary Aspects of Time and Time Perception. Lecture Notes in Computer Science Vol. 6789. Berlin: Springer. doi: 10.1007/978-3-642-21478-3

Vatakis, A., \& Ulrich, U. (2014). Temporal processing within and across senses. Acta Psychologica, Volume 147 and special section of Volume 149.

Viau-Quesnel, C., Gaudreault, R., Ouellet, A-A., \& Fortin, C. (2014). Making sense of timing and attention: Modality effect in timing with a break. Timing \& Time Perception, 2, 129-144. doi: 10.1163/22134468-00002019

Vicario, C. M. (2011). Perceiving numbers affects the subjective temporal midpoint. Perception, 40, 23-29. doi: $10.1068 / p 6800$

von Mühlenen, A., \& Lleras, A. (2007). No-onset looming motion guides spatial attention. Journal of Experimental Psychology: Human Perception and Performance, 33(6), 1297-1310. doi: 10.1037/0096-1523.33.6.1297

Vuillleumier, P., \& Driver, J. (2007). Modulation of visual processing by attention and emotion: Windows on causal interactions between human brain regions. Philosophical Transactions of the Royal Society B, 362, 837-855. doi: 10.1098/rstb.2007.2092

Wackermann, J., \& Ehm, W. (2006). The dual klepsydra model of internal time representation and time reproduction. Journal of Theoretical Biology, 239, 482-493. doi:10.1016/j.jtbi.2005.08.024

Walker J. T., \& Scott, K. J. (1981). Auditory-visual conflicts in the perceived duration of lights, tones and gaps. Journal of Experimental Psychology: Human Perception and Performance, 7, 13271339.

Walsh, V. (2003). A theory of magnitude: Common cortical metrics of time, space and quantity. Trends in Cognitive Sciences, 7, 483-488. doi:10.1016/j.tics.2003.09.002

Wang, L., \& Jiang, Y. (2012). Life motion signals lengthen perceived temporal duration. Proceedings of the National Academy of Sciences USA, 109(11), E673-E677. doi: 10.1073/pnas.1115515109

Warm, J. S., Greenberg, L. F., \& Dube, C. S. (1964). Stimulus and motivational determinants of temporal perception. Journal of Psychology, 58, 243-248.

Warm, J. S., McCray, R. E. (1969). Influence of word frequency and length on apparent duration of tachistoscopic presentations. Journal of Experimental Psychology, 79, 56-58.

Watanabe, J., Amemiya, T., Nishida, S., \& Johnston, A. (2010). Tactile duration compression by vibrotactile adaptation. NeuroReport, 21(13), 856-860. doi: 10.1097/WNR.0b013e32833d6bcb

Wearden, J. H. (1992). Temporal generalization in humans. Journal of Experimental Psychology: Animal Behavior Processes, 18, 134-144.

Wearden, J.H. (2004). Decision processes in models of timing. Acta Psychologica, 64, 303-317.

Wearden, J. H., Edwards, H., Fakhri, M., \& Percival, A. (1998). Why "sounds are judged longer than lights": Application of a model of the internal clock in humans. Quarterly Journal of Experimental Psychology, 51B, 97-120. 
Wearden, J. H., \& Ferrara, A. (1995). Stimulus spacing effects in temporal bisection by humans. Quarterly Journal of Experimental Psychology, 48B, 289-310.

Wearden, J. H., \& Jones, L. A. (2007). Is the growth of subjective time in humans a linear or nonlinear function of real time? Quarterly Journal of Experimental Psychology, 60, 1289-1302.

Wearden, J. H., \& Lejeune, H. (2008). Scalar properties in human timing: Conformity and violations. Quarterly Journal of Experimental Psychology, 61, 569-587.

Wearden, J. H., Norton, R., Martin, S., \& Montford-Bebb, O. (2007). Internal clock processes and the filled-duration illusion. Journal of Experimental Psychology: Human Perception and Performance, 33(3), 716-729.

Wearden, J. H., \& Penton-Voak, I. S. (1995). Feeling the heat: Body temperature and the rate of subjective time, revisited. Quarterly Journal of Experimental Psychology, 48B(2), 129-141. doi: 10.1080/14640749508401443

Wearden, J. H., Todd, N. P. M., \& Jones, L. A. (2006). When do auditory/visual differences in duration judgements occur? Quarterly Journal of Experimental Psychology, 59(10), 1709-1724. doi: $10.1080 / 17470210500314729$

Whittlesea, B. W. A. (1993). Illusions of familiarity. Journal of Experimental Psychology: Learning Memory and Cognition, 19, 1235-1253.

Wilkins, A., Nimmo-Smith, I., Tait, A., McManus, C., Della Sala, S., Tilley, A., ...Scott, S. (1984). A neurological basis for visual discomfort. Brain, 107, 989-1017. doi: 10.1093/brain/107.4.989 989-1017

Witherspoon, D., \& Allan, L. G. (1985). The effect of a prior presentation on temporal judgments in a perceptual identification task. Memory \& Cognition, 13, 101-111.

Wittmann, M. (2013). The inner sense of time: How the brain creates a representation of duration. Nature Reviews Neuroscience, 14, 217-223. doi:10.1038/nrn3452

Xuan, B., Zhang, D., He, S., Chen, X. C. (2007). Larger stimuli are judged to last longer. Journal of Vision, 7(1), Article 2, 1-5.

Yamada, Y., \& Kawabe, T. (2011). Emotion colors time perception unconsciously. Consciousness and Cognition, 20, 1835-1841. doi: 10.1016/j.concog.2011.06.016

Yamamoto, K., \& Miura, K. (2012). Perceived duration of plain motion increases with pattern speed rather than component speed. Journal of Vision, 12(4), Article 1, 1-13. doi: 10.1167/12.4.1.

Yates, M. J., Loetscher, T., \& Nicholls, M. E. R. (2012). A generalized magnitude system for space, time, and quantity? A cautionary note. Journal of Vision, 12(7). Article 9. 1-7. doi: 10.1167/12.7.9.

Yerkes, R. M., \& Urban, F. M. (1906). Time-estimation in its relations to sex, age, and physiological rhythms. Harvard Psychological Studies, 2, 405-430.

Yeshurun, Y., \& Levy, L. (2003). Transient spatial attention degrades temporal resolution. Psychological Science, 14, 225-231.

Yeshurun, Y., \& Marom, G. (2008). Transient spatial attention and the perceived duration of brief visual events. Visual Cognition, 16, 826-848.

Yiend, J. (2010). The effects of emotion on attention: A review of attention processing of emotional information. Cognition and Emotion, 24(1), 3-47. doi: 10.1080/02699930903205698

Yin, B., Lusk, N. A., \& Meck, W. H. (in press). Interval-timing protocols and their relevancy to the study of temporal cognition and neurobehavioral genetics. In V. Tucci (Ed.) Neuro-phenome: Cutting-edge approaches and technologies in neurobehavioral genetics. Wiley-Blackwell.

Yu, A. C. L. (2010). Tonal effects on perceived vowel duration. Laboratory Phonology, 10, 151-168 
Zakay, D. (1998). Attention allocation policy influences prospective timing. Psychonomic Bulletin \& Review, 5(1), 114-118.

Zakay, D., \& Block, R. A. (1997). Temporal cognition. Current Directions in Psychological Science, 6, 12-16.

Zelkind, I. (1973). Factors in time estimation and a case for the internal clock. Journal of General Psychology, 88, 295-301.

Zhou, B., Yang, S., Mao, L., \& Han, S. (2014). Visual feature processing in the early visual cortex affects duration perception. Journal of Experimental Psychology: General, 143, 1893-1902. doi: 10.1037/a0037294 

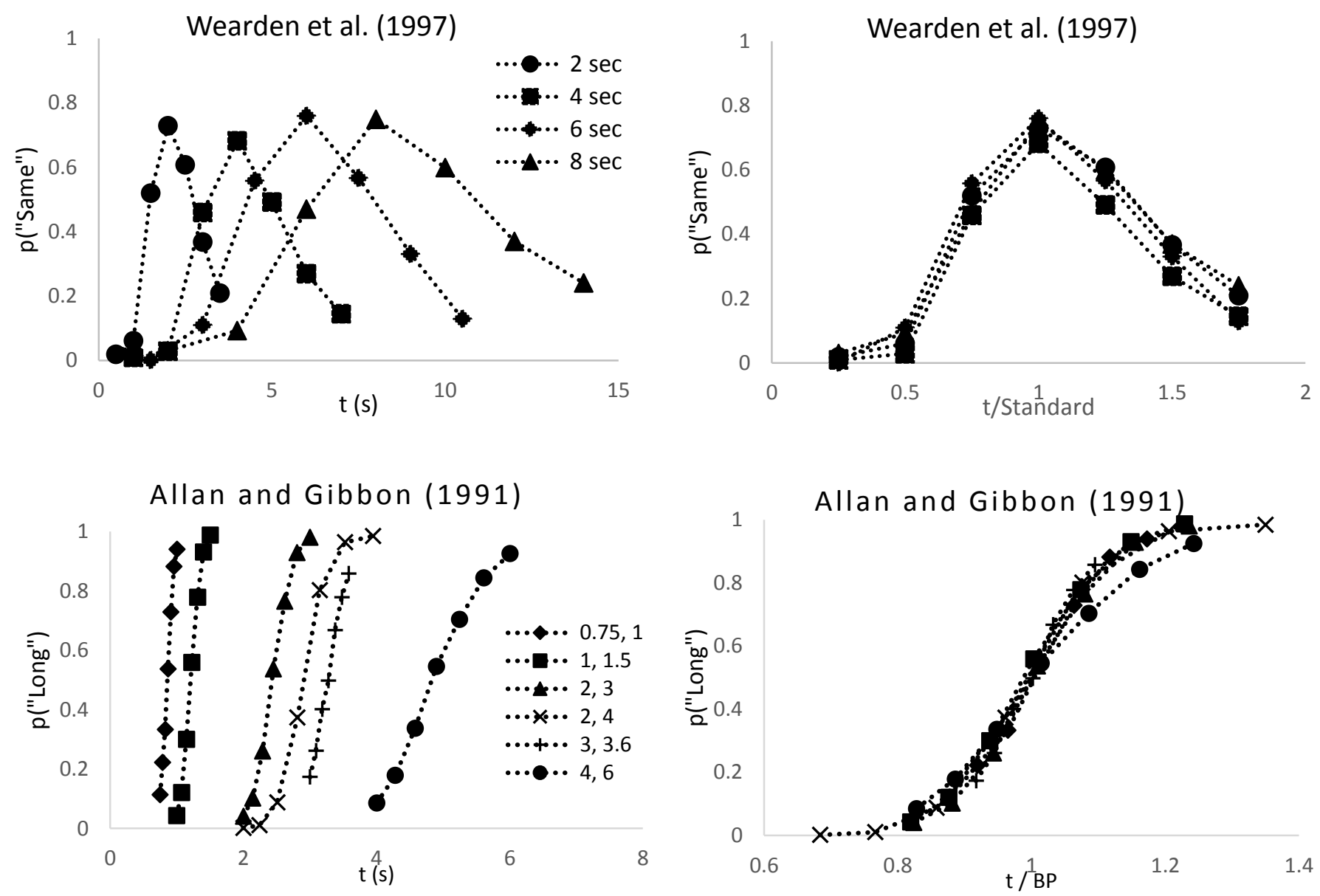

Figure 1. Illustrations of time-scale invariance. The top-left panel plots data from a temporal generalization task in which participants classified tones as "same" or "different" from an encoded standard duration. Each line represents performance with a different standard. The top-right panel plots the same data after dividing the test durations by the standard duration; the generalization gradients superimpose, illustrating scale invariance. (Adapted from Wearden et al., 1997). The bottom-left panel shows data from a task in which participants classified durations as closer to "short" or "long" anchor durations; each line indicates a different pair of anchors (e.g., 0.75, $1=$ short anchor was 0.75 seconds, long anchor was 1.0 seconds). The bottom-right panel plots the same data when the stimulus durations have been normalized by the relevant bisection point. Again, there is good superimposition. (Adapted from Allan \& Gibbon, 1991.) 


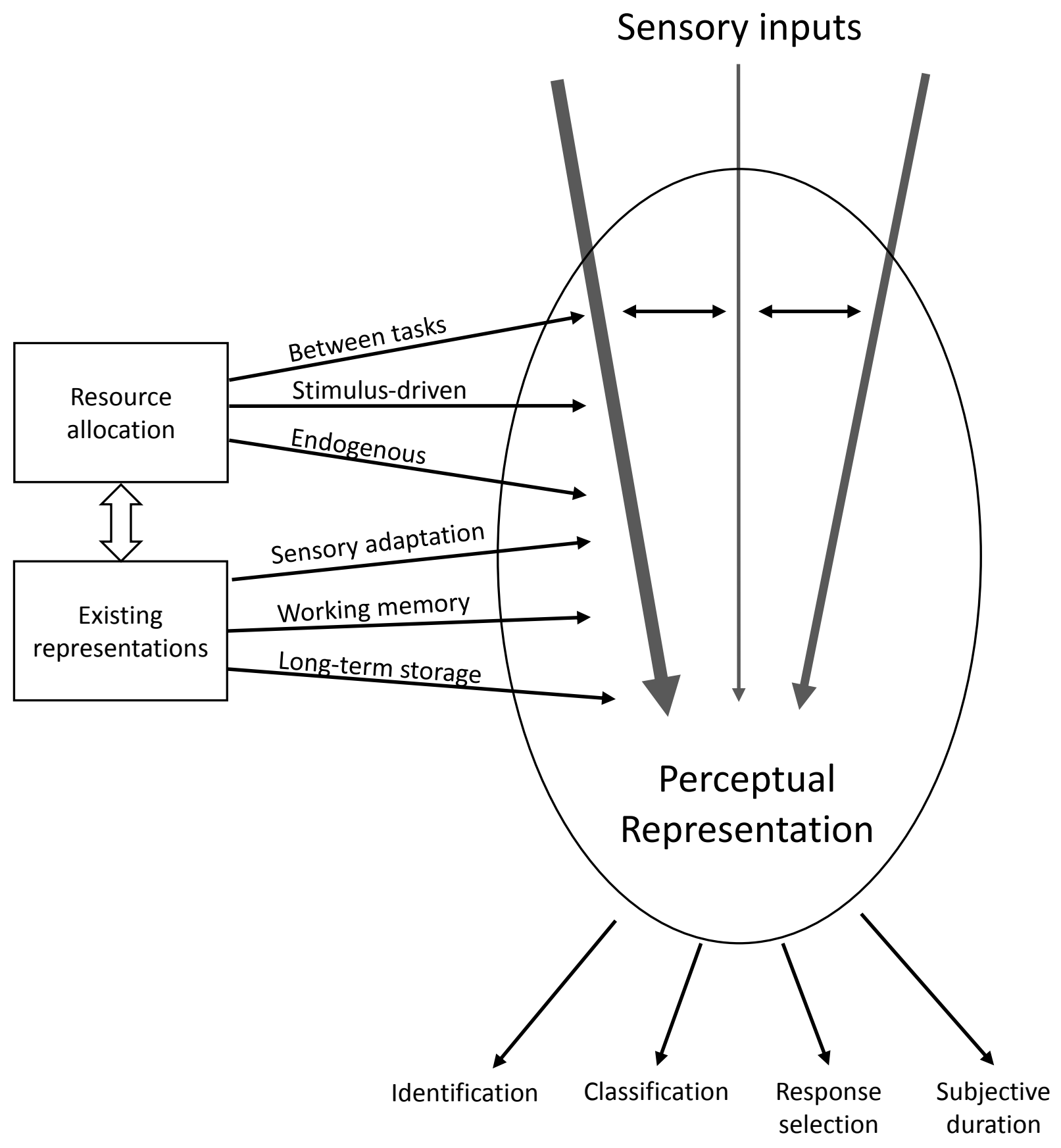

Figure 2. The processing principle. Perceptual representations depend on the confluence of external stimulation and internal processing. Incoming sensory information may differ in intrinsic signal strength (indicated by the differing widths of the input arrows) but will also be moderated by relations between the inputs (indicated by horizontal arrows), by the allocation of processing capacity (attention), and by the presence of existing stimulus representations (memory). These stimulus, attentional, and mnemonic factors will be inter-related, and their inter-play determines the effective clarity of the final percept - the vividity of the representation and the ease with which information can be extracted from it to make decisions about stimulus identity, category membership, and so forth. As an empirical generalization, the conditions that promote perceptual clarity correspond to expanded subjective duration, a regularity that we label the processing principle. 

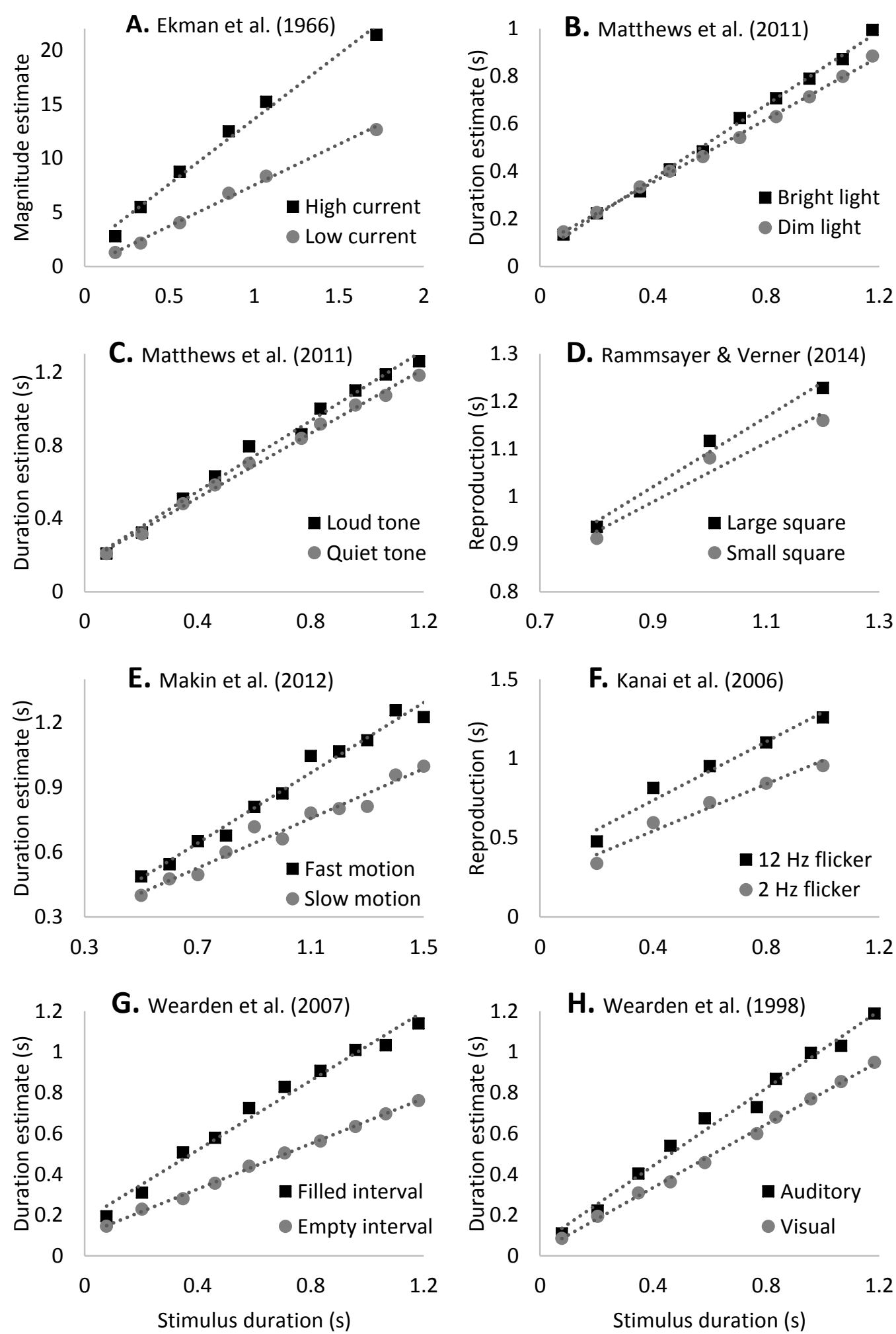

Figure 3. Some examples of how basic perceptual properties affect subjective time. Panel A: Subjective duration is longer for more intense electrical stimulation. The plot compares temporal magnitude estimates for periods of electrical stimulation of the skin at low intensity (1.5 times threshold) and high intensity (3.5 times threshold) (Adapted from Ekman et al., 1966). Panel B: Against a dark background, verbal estimates of duration are greater for bright lights bright (134 $\mathrm{cd} / \mathrm{m}^{2}$ ) than for $\operatorname{dim}$ ones $\left(0.58 \mathrm{~cd} / \mathrm{m}^{2}\right)$. (Adapted from Matthews et al., 2011). Panel C: for tones 
presented in silence, verbal duration estimates are larger for loud ( $80 \mathrm{~dB}$ ) than for quiet ( $59 \mathrm{~dB})$ stimuli. (Adapted from Matthews et al., 2011). Panel D: mean reproductions are larger for large (10.0 degrees) squares than for small (1.2 degrees) squares. (Data from Rammsayer \& Verner, 2014). Panel E: Verbal duration estimates are longer for drifting gratings that move fast ( 15 degrees $/ \mathrm{s}$ ) than for those that move slowly ( 5 degrees/s). (Adapted from Makin et al., 2012). Panel F: Reproduced durations were longer for squares that flicker at high frequency $(12 \mathrm{~Hz})$ than for those flickering at low frequency (2 Hz). (Data from Kanai et al., 2006). Panel G. Verbal estimates are longer for intervals filled with a continuous tone than for silent intervals demarcated by two brief clicks. (Adapted from Wearden et al., 2007). Panel $\mathrm{H}$. Verbal estimates for auditory stimuli (here a $500-\mathrm{Hz}$ tone) are often longer than those for visual stimuli (here a blue square), although this effect may well depend on the specific auditory/visual stimuli that are compared (see main text). (Adapted from Wearden et al., 1998). 
A

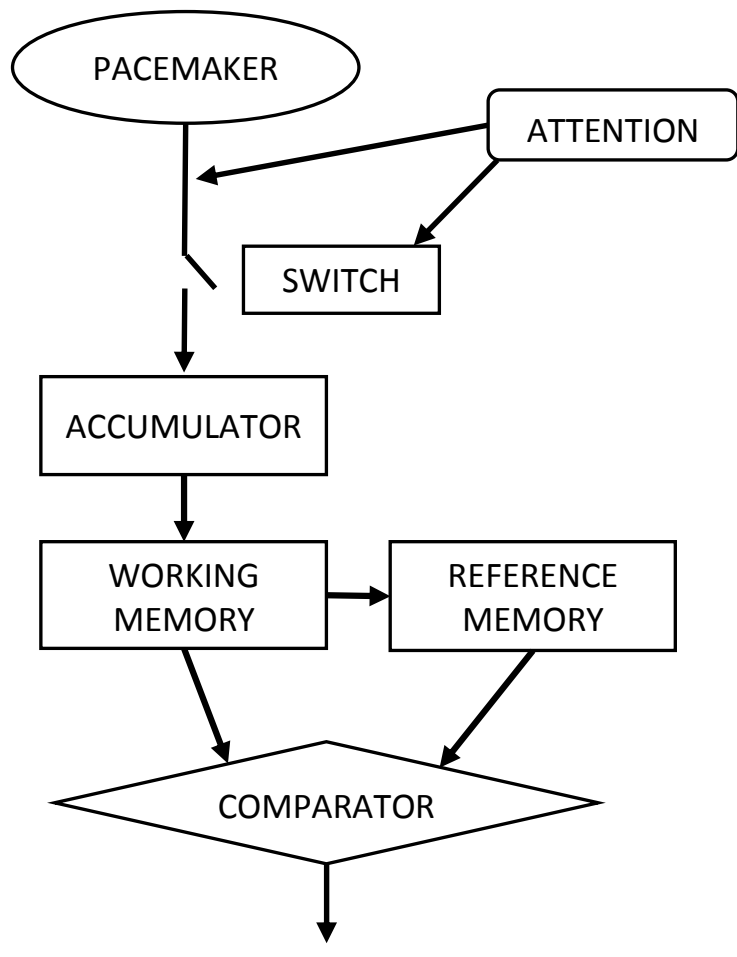

BEHAVIOUR

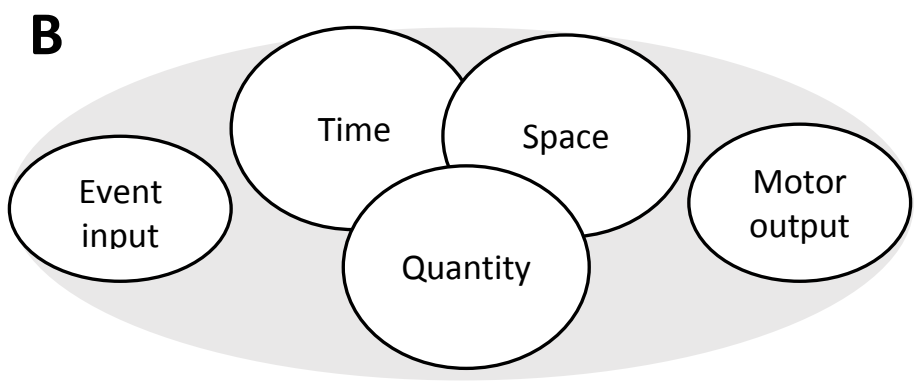

C

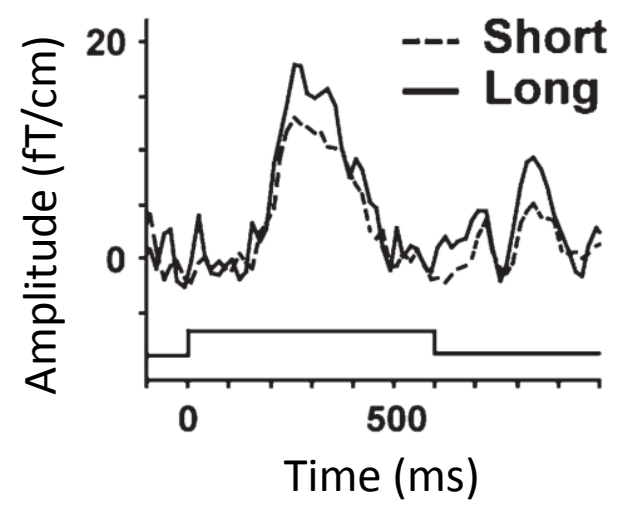

Figure 4. Three models for time perception. (A) A generic pacemaker-accumulator framework (modelled after Gibbon et al., 1984; Treisman, 2013; Wearden, 2004). A dedicated pacemaker emits pulses; timing begins when a switch closes, allowing the pulses to flow into an accumulator, and ends when the switch opens again. Both the effective flow of pulses and the operation of the switch may depend on sustained attention to the stimulus. The accumulated pulses form the representation of subjective duration and may be transferred to working memory and thence to long-term storage (not all models posit separate working memory and accumulator components). Temporal decisions are based on comparison of the working memory representation with previously-encoded pulse-counts. Non-temporal stimulus properties may affect subjective duration by altering the rate of the pacemaker and/or the latency of the switch. (B) A "common metric" framework in which time, space, and quantity (including symbolic numbers) have shared neural representations, perhaps in the parietal cortex, as the result of the relevance of these dimensions for action. Subjective time is therefore inextricably linked to the measurement of non-temporal magnitude. (Adapted from Walsh, 2003). (C) A coding-efficiency account. Subjective time may be directly related to the total energy expended encoding the stimulus, such that stimulus properties which evoke a larger neural response have longer apparent duration. The figure plots data from a magnetoencephalography (MEG) study by Noguchi and Kakigi (2006) and shows how the evoked response was larger for stimuli that were classified as "long" than for those judged "short", despite identical physical durations. 

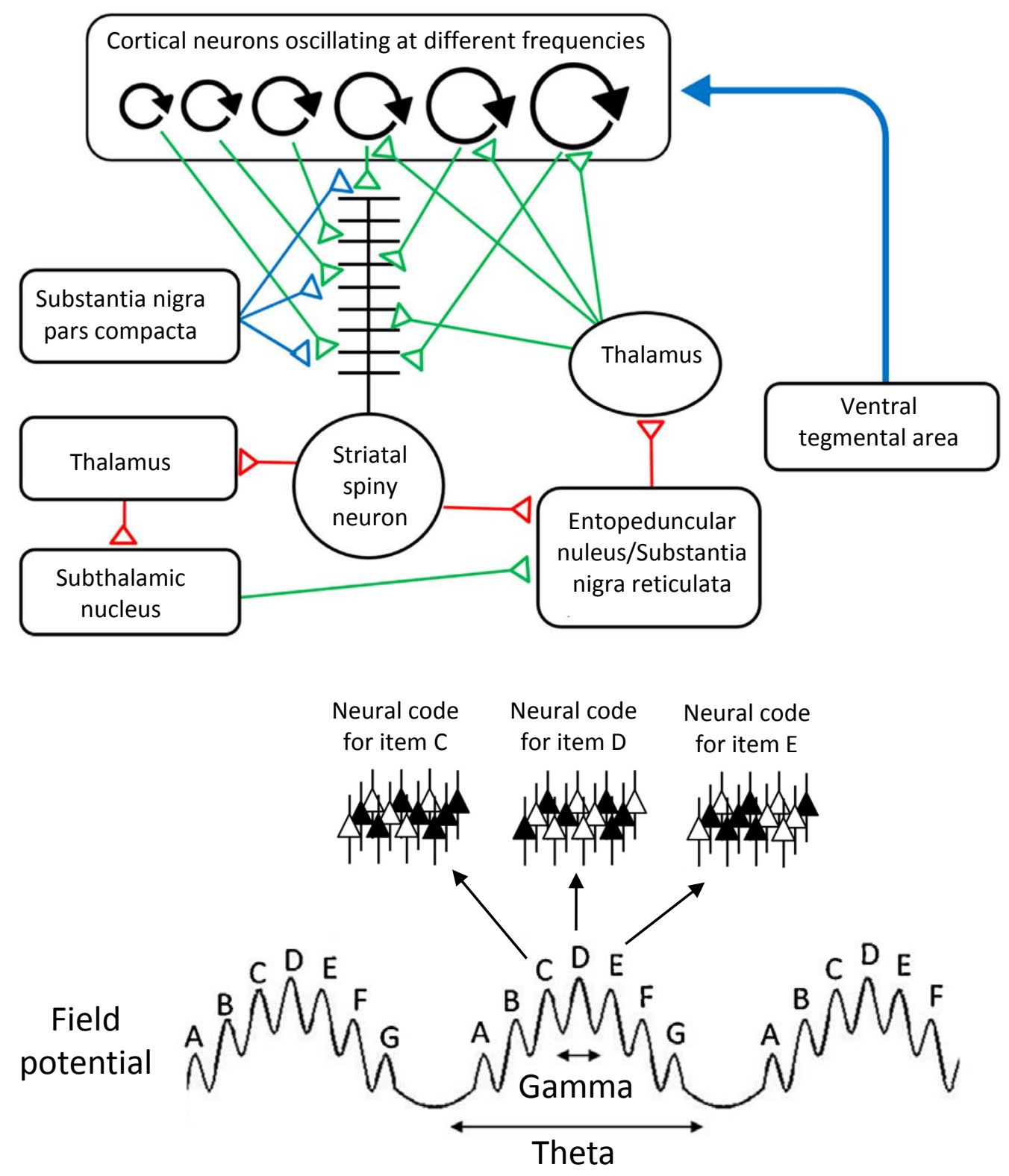

Figure 5. Cortical oscillations as the basis for temporal and identity information. The top panel shows the striatal beat frequency model. Cortical neurons oscillating at different frequencies project on to striatal spiny neurons. The set of oscillators which are active at the time of a relevant signal (e.g., stimulus offset) provide the code for duration. The lower panel illustrates how theta and gamma cortical oscillations may also underlie the maintenance of stimulus representations in working memory: individual items are encoded in the spatial pattern of cells firing within a given gamma cycle, with the whole sequence repeated on subsequent theta cycles - and with the capacity of short-term memory dependent on the number of items that can be "fit" into each theta cycle. Recent modelling has shown that working memory representations and timing can be encoded in a single oscillator-based framework, with different dimensions of the neural oscillations providing the basis for item, order, and duration information. Adapted from Gu et al. (2015). 

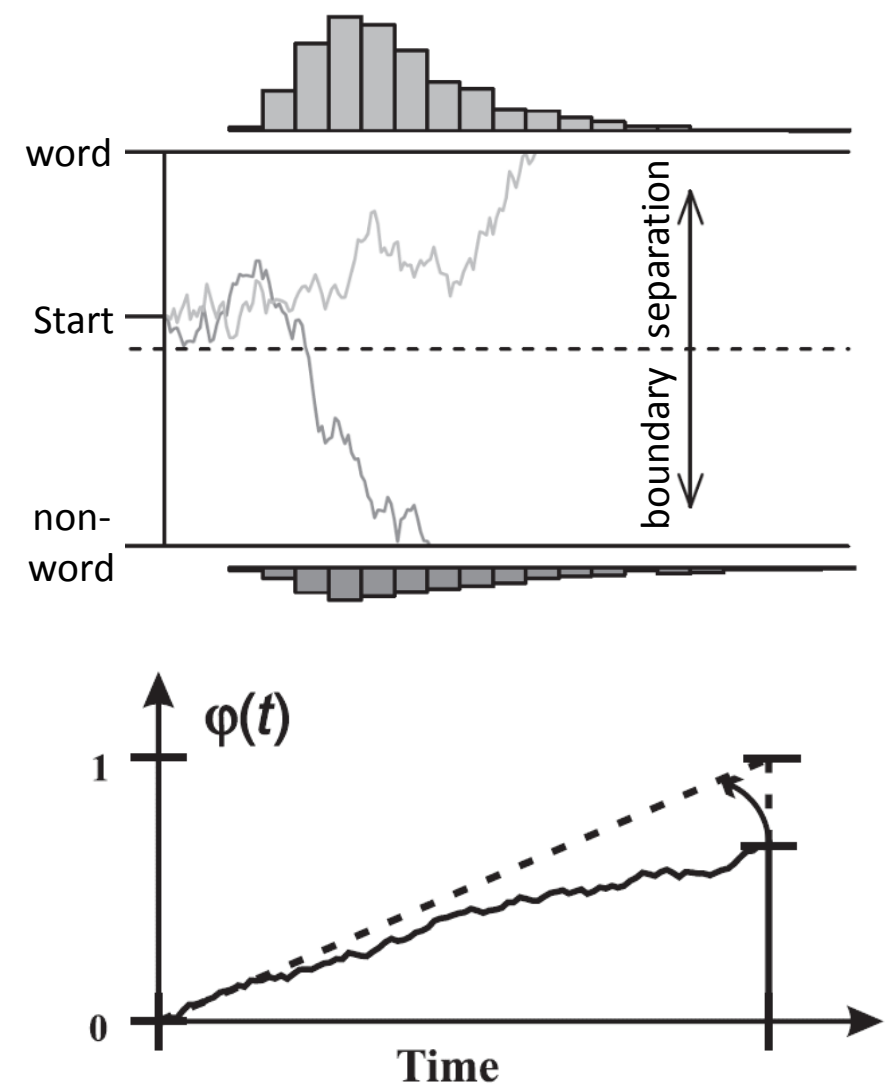

Figure 6. Drift-diffusion models of perceptual decision-making and timing. The top panel illustrates a drift-diffusion model of two-alternative decision-making. Each irregular line represents one iteration of the decision process, a time-continuous random walk that terminates when it reaches one of two absorbing barriers corresponding to the response alternatives (e.g., "word" and "non-word"). The histograms show the distribution of first-passage times for the two barriers which, in combination with the time taken for non-decisional processes, will determine the RTs for each type of response. Stronger perceptual evidence entails reduced noise and a higher mean drift rate, such the process will be more likely to reach the correct barrier (higher accuracy) and will take less time to do so (shorter RTs). (Adapted from Dutilh, Krypotos, \& Magenmakers, 2011). The bottom panel illustrates a time-adaptive drift diffusion model (TDDM) of interval timing. The distance from the start provides the measure of time, and there is a single, fixed absorbing barrier. Learning to time an interval entails adjusting the drift rate such that the barrier is reached after the appropriate physical time has elapsed (indicated by the curved arrow showing the adjustment that would be made for the next trial). (Adapted from Luzardo, Ludvig, \& Rivest, 2013). 

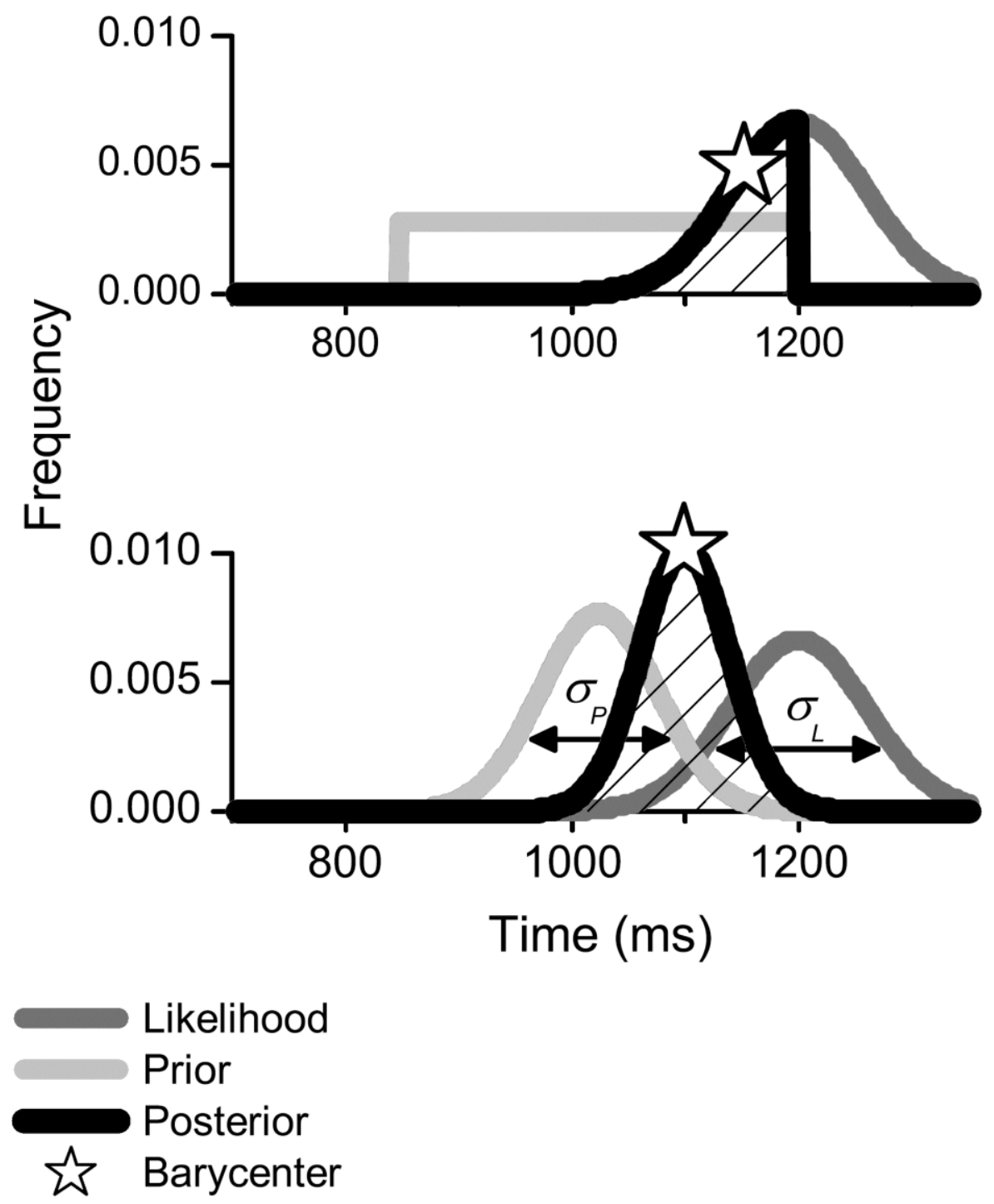

Figure 7. "Contextual calibration" of time estimates. The top panel shows the ideal observer model of Jazayeri and Shadlen (2010) in which the Gaussian likelihood function for the current duration (dark gray line) is combined with a uniform prior distribution of durations experienced during the test session (light gray line) to obtain a posterior distribution (black line). In this account, the prior provides perfect information about the range of experienced durations, assigning zero weight to durations outside that range. The centre of mass of the posterior distribution (Barycenter) provides the observer's estimate of the stimulus. The bottom panel shows a refined model in which the prior is itself a Gaussian distribution, reflecting uncertainty about the stimulus distribution; the form of this prior can differ between observers and conditions (Cicchini et al., 2012). Adapted from Cicchini et al. (2012). 